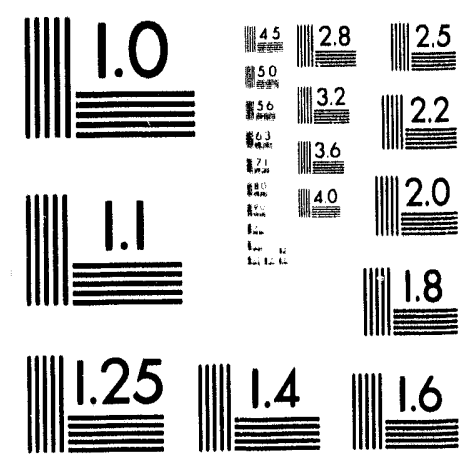



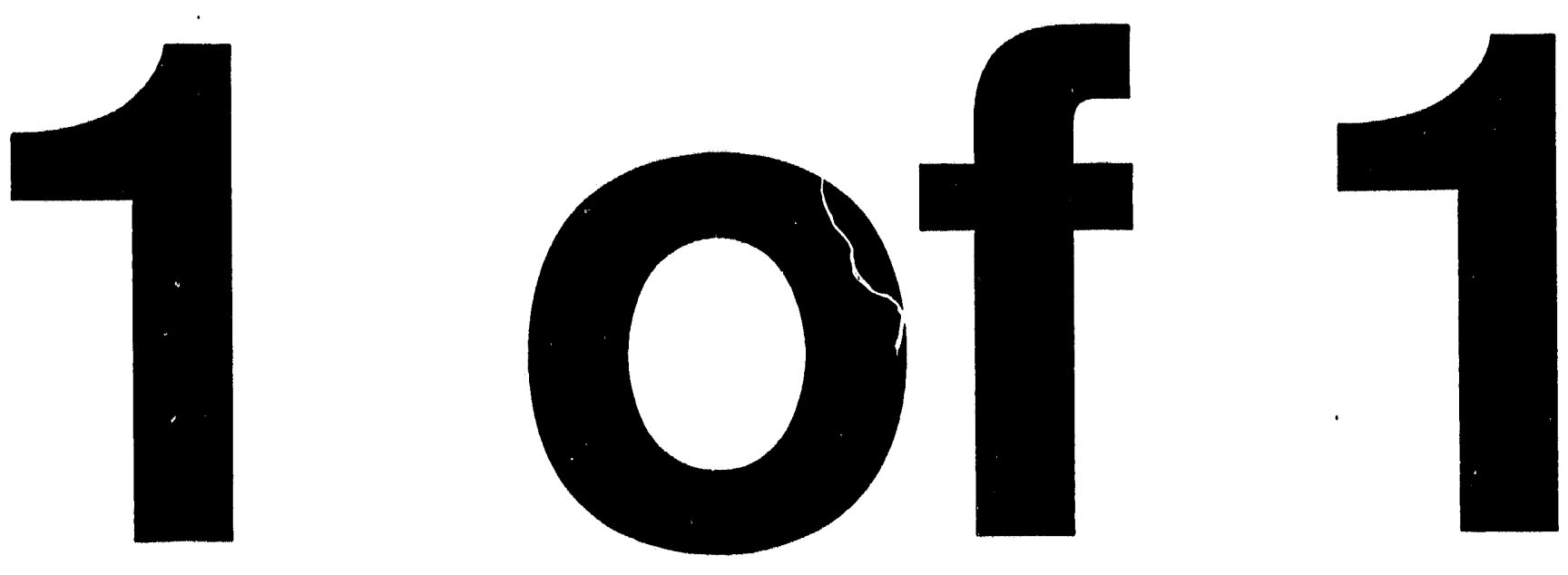


\section{DEVELOPMENT OF A CONTINUOUS PROCESS FOR ADJUSTING NITRATE, ZIRCONIUM, AND FREE HYDROFLUORIC ACID CONCENTRATIONS IN ZIRCONIUM FUEL DISSOLVER PRODUCT}

D. A. Cresap

D. S. Halverson

Aprll 1993

Westinghouse Idaho

PREPARED FOR THE

DEPARTMENT OF ENERGY

IDAHO OPERATIONS OFFICE

UNDER CONTRACT DE-AC07-841D12435

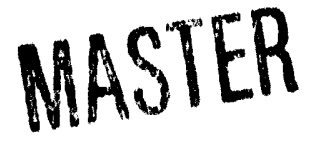




\section{Acknowledgement}

The authors gratefully acknowledge the assistance of James A. Murphy in providing the HF probe equations and original feedforward complexing equations. 
TABLE OF CONTENTS

SUMMARY . . . . . . . . . . . . . . 1

1.0 INTRODUCTION . . . . . . . . . . . . . . . . . . . . . . . . 2

2.0 PROGRAM OBJECTIVES ................... 3

3.0 EQU!PMENT . . . . . . . . . . . . . . . . . . . . 3

4.0 CONTROL SYSTEM ..................... 8

4.1 Current Status .................... 8

4.2 Development History ............... 8

5.0 RESULTS AND DISCUSSION ....................... 11

5.1 HF Target Attainment .............. 11

5.2 Probe Operation and Reliability.......... 18

5.3 Zr Target Attainment ............... 22

5.4 Nitrate Target Attainment . . . . . . . . . . 27

5.5 Corrosion ................. 28

6.0 RECOMMENDATIONS FOR FUTURE WORK ............... 28

7.0 CONCLUSIONS .................... 32

REFERENCES .......................... 33

Appendix A MICROMAX Description and Configuration . . . . . . . . A-1

TABLES

TABLE $2.0-1 \ldots \ldots \ldots 4$

TABLE $5.1-1 \ldots \ldots \ldots$

FIGURES

FIGURE 2.0-1 ILCM SYSTEM . . . . . . . . . . . . . . . . 5

FIGURE 2.0-2 HP PROBE RECIFCULATION LOOP . . . . . . . . . . . . . . . . . 6

FIGURE 2.0-3 HF PROBE . . . . . . . . . . . . . . . . . 7

FIGURE 5.1-1 PERCENT DEVIATION OF COMPOSITE FREE HF CONCENTRATION FOR

ALL ILCM RUNS . . . . . . . . 12

Figure 5.1-2 FREE HF READING AT TUBE i DURING RUN 3 VERSUS TIME....$\quad 13$

Figure 5.1-3 Free HF READING AT TUBE I DURING RUN 5 VERSUS TIME .... . 15

Figure 5.1-4 Free HF Reading AT TUBE I DURING RUN 6 VERSUS TIME . . . . 16

FIGURE 5.1-5 FREE HF READING AT TUBE I DURING RUN 8 VERSUS TIME ..... 17

Figure 5.2-1 COMPARISON OF COMPUTER CALCULATED FREE HF CONCENTRATIONS

TO THE CORRESPONDING HF PROBE READINGS . . . . . . . 21

Figure 5.3-1 ZIRCONIUM TARGET ATTAINME IT . . . . . . . . . . . . . . . . 24

Figure 5.3-2 COMPARISON OF RAL'S ZIRCONIUM ANALYSIS TO THE SYSTEM MASS

BALANCE RESULTS . . . . . . . . . . . . . . . . 25

Figure 5.4-1 NITRATE TARGET ATTAINMENT 29

Figure 5.4-2 COMPARISON OF THE RAL'S NITRATE ANALYSIS TO THE SYSTEM MASS

BALANCE RESULTS ................ 30 
SUMMARY

In the Fluorinel Dissolution Process (FDP) upgrade, excess hydrofluoric acid in the dissolver product must be complexed with aluminum nitrate (ANN) to eliminate corrosion concerns, adjusted with nitrate to facilitate extraction, and diluted with water to ensure solution stability. This is currently accomplished via batch processing in large vessels. However, to accommodate increases in projected throughput and reduce waste production in a costeffective manner, a semi-continuous system (In-line Complexing (ILC)) has been developed. The major conclusions drawn from tests demonstrating the feastbility of this concept are given below.

The ILCM consistently approached the control target of $0.045 \mathrm{M}$ free HF (error range $-14 \%$ to $8 \%$.) In addition, the necessary residence time for the reaction appeared to be between 0.3 and 0.67 minutes. The titanium probe used to amperometrically and continuously the HF concentration had an adequate life for development tests. Additional work is suggested to develop a probe that will have an adequate life for plant operation.

The tests indicated that two points for ANN addition are adequate. During the last five runs of the system, complexing was essentially complete through the use of the first ANN addition point only. However, the second addition system may be required in the event of extreme upset conditions or fallure of the first addition system.

The ILCM also consistently approached its nitrate (error range -7 to $13 \%$ ) and zirconium ( $Z r$ ) (error range -16 to $12 \%$ ) targets in the absence of equipment failure. The primary problem with attaining these targets was accurate reagent addition. Another source of error in the final $\mathrm{Zr}$ concentration was the density equation used to estimate the initial $\mathrm{Zr}$ concentration. Since these errors are hardware related, and the equipment which will be used in the plant will not be identical to that used in the mock-up, it will be necessary to test the actual plant hardware in the integrated pilot plant to ensure that these problems can be resolved. 
A test of the system carried out with a mixture of ANN and nitric acid demonstrated the applicability of the process to complexing $2 r$ dissolver product with Al fuel dissolver product (coprocessing).

The control system was tuned to provide stable operation during the last run of the system. In addition, the system was able to attain the HF target within 10 minutes with a decay ratio of 0.3 and a period of oscillation of 3.5 minutes after the initial start-up disturbance. It is posstble that smoothing of the incoming HF probe signal coupled with derivative control could improve the system's abllity to deal with disturbances. In addition, the magnitude of the initial start-up disturbance could be reduced via closer tuning of the feedforward contribution.

The system tests were too short to obtain any information on the corrosive effects of HF/HNO3 mixing in the system.

\subsection{INTRODUCTION}

In the FDP upgrade, excess hydrofluoric acid in the dissolver product (ZrDP) must be complexed with aluminum nitrate to eliminate corrosion concerns, adjusted with nitrate (nitric acid) to facilitate extraction, and diluted with water to ensure solution stability of zirconium fluoride. These operations are integrated, that is, adjustment of concentration of one affects the other. The CPP currently uses a batch process for this purpose. The large vessels, instrumentation, and cell space associated with this complexing technique are expensive. The cost savings associated with the reduced requirements for cell space which would result from continuous complexing is estimated to be $\$ 60,000,000$. In addition, the current batch process is not geometrically safe by design and requires the use of nuclear poisons for criticality control resulting in increased waste volumes. The batch process also involves mixing and sampling as separate steps, with significant dead time associated with sample result turnaround. In addition, the reagent additions are imprecise, resulting in a frequent need for adjustment, increasing waste volume and delaying plant operation. 
In order to solve these problems, the authors developed a continuous in-line complexing system to complex the zirconium dissolver product ( 2 rDP) as an alternative to batch processing. By using a $\mathrm{Ti}$ probe to detect free $\mathrm{HF}$ concentrations ${ }^{17}$, along with equations which enable the extent of complexing to be estimated, the In-line Complexing System (ILCS) is able to use a feedforward/feedback cascaded control scheme to continuousiy attain the free HF target concentration. The system al so uses feedforward control to simultaneously attain $\mathrm{Zr}$ and nitrate target concentrations.

\subsection{PROGRAM OBJECTIVES}

- Develop a process and control system which continuously attains the $\mathrm{HF}, \mathrm{Zr}$, and nitrate targets required during batch complexing.

Determine the residence time required for the complexing reaction.

- Determine the reliability and operating characteristics of the probes.

Demonstrate the system's applicability to coprocessing.

Investigate the corrosion effects of HF/Nitric acid mixing in the system.

Table 2.0-1 lists the test conditions and targets used for all of the ILCM system runs.

\subsection{EQUIPMENT}

The mock-up was installed in the Geometrically Favorable Dissolver (GFD) module in the Low Bay Laboratory and used the Fountain Dissolver Mockup (FDM) recycle vessel (operating capacity of 195 liters) as the uncomplexed ZrDP 
TABLE 2.0-1

\begin{tabular}{|c|c|c|c|c|c|c|c|}
\hline \multirow[b]{2}{*}{$\begin{array}{l}\text { Run Number } \\
\text { (Date) }\end{array}$} & \multirow[b]{2}{*}{$\begin{array}{c}\text { ZrDP } \\
\text { Flowrate } \\
\text { (liters/min) }\end{array}$} & \multicolumn{5}{|c|}{ EXOERIMENTAL CONDITIONS AND TARGETS } & \multirow[b]{2}{*}{$\begin{array}{l}\text { RAL } * \mathrm{NO}_{3} \\
\text { Analyele } \\
\text { Error } \\
( \pm x)\end{array}$} \\
\hline & & Temp. ${ }^{\circ} \mathrm{C}$ & $\begin{array}{l}\text { Froe } H F \\
\text { Target" } \\
\text { (molar) }\end{array}$ & $\begin{array}{c}2 r \\
\text { Target } \\
\text { (molar) }\end{array}$ & $\begin{array}{l}\text { TSL } 2 r \\
\text { Analysis } \\
\text { Error } \\
( \pm x)\end{array}$ & $\begin{array}{l}\text { Nitrote } \\
\text { Target } \\
\text { (noler) }\end{array}$ & \\
\hline $\begin{array}{c}\text { ILCNOO1 } \\
(7 / 18 / 90) \\
\end{array}$ & $2 \cdot 2.25$ & $2 \bar{c}$ & 0.045 & 0.5 & 9 & 2.0 & 10 \\
\hline $\begin{array}{c}\text { ILCM002 } \\
(7 / 25 / 90)\end{array}$ & 1.5 & 22 & 0.045 & 0.5 & 9 & 2.3 & 18 \\
\hline $\begin{array}{c}\text { ILCMO03 } \\
(8 / 30 / 90) \\
\end{array}$ & 9.5 & 21 & 0.045 & 0.5 & 10 & 2.3 & 11 \\
\hline $\begin{array}{l}\text { ILCMOOL } \\
(9 / 20 / 90) \\
\end{array}$ & 1.0 & 70 & 0.045 & 0.5 & 9 & 2.4 & 11 \\
\hline $\begin{array}{c}\text { ILCMOOS } \\
(11 / 20 / 90)\end{array}$ & $1 \cdot 1.5$ & $75 \cdot 83$ & 0.045 & 0.5 & 15 & 2.4 & 6 \\
\hline $\begin{array}{l}\text { ILCMOOS } \\
(3 / 21 / 90)\end{array}$ & $1 \cdot 1.5$ & $77 \cdot 82$ & 0.045 & 0.5 & 3 & 2.4 & 6 \\
\hline $\begin{array}{c}\text { ILCMOO7 } \\
(5 / 21 / 91) \\
\end{array}$ & ABORTED & & & & & & \\
\hline $\begin{array}{c}\text { ILCMO08 } \\
(5 / 29 / 91)\end{array}$ & $1 \cdot 4.0$ & 22 & 0.045 & 0.5 & 10 & 2.4 & 7 \\
\hline $\begin{array}{l}\text { Colcu } \\
\text { tempe } \\
\text { Based }\end{array}$ & $\begin{array}{l}\text { d at } 35^{\circ} \mathrm{C} \text {; } \\
\text { re. } \\
\text { two standare }\end{array}$ & & eet $v$ & oper & & dep & nt on the \\
\hline
\end{tabular}

supply. The recycle vessel was equipped with density, level and temperature monitoring instrumentation. These readings were used to estimate the zirconium concentration in the recycle vessel prior transfer from the tank into the in-line complexing system. This concentration was then used in conjunction with the flow rate from the ZrDP supply pump in determining the feedforward contribution to the flow rates for the other reagents.

A sketch of the In-line Complexing Mock-up is shown in Figure 2.0-1. The system consists of a supply vessel containing the uncomplexed dissolver product, a series of six 4" I.D. Kynar tubes connected by I" Kynar tubing, recycle loops (see figure 2.0-2), HF probes (see Figure 2.0-3), thermocouples, metering pumps, in-line mixers, complexing reagent addition lines, and a final collection vessel. 


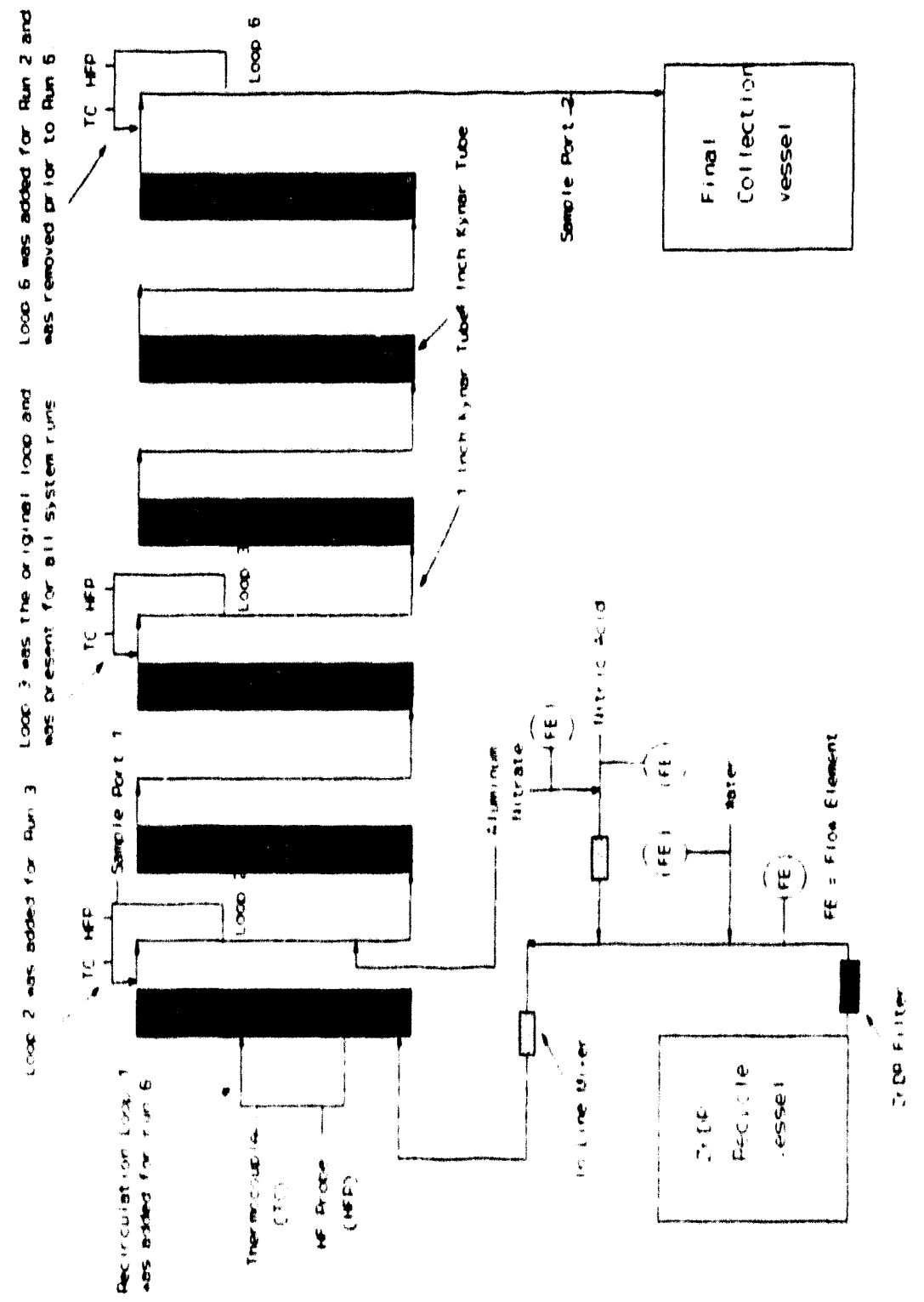

FIGURE 2.0-1 ILCM SYSTEM 


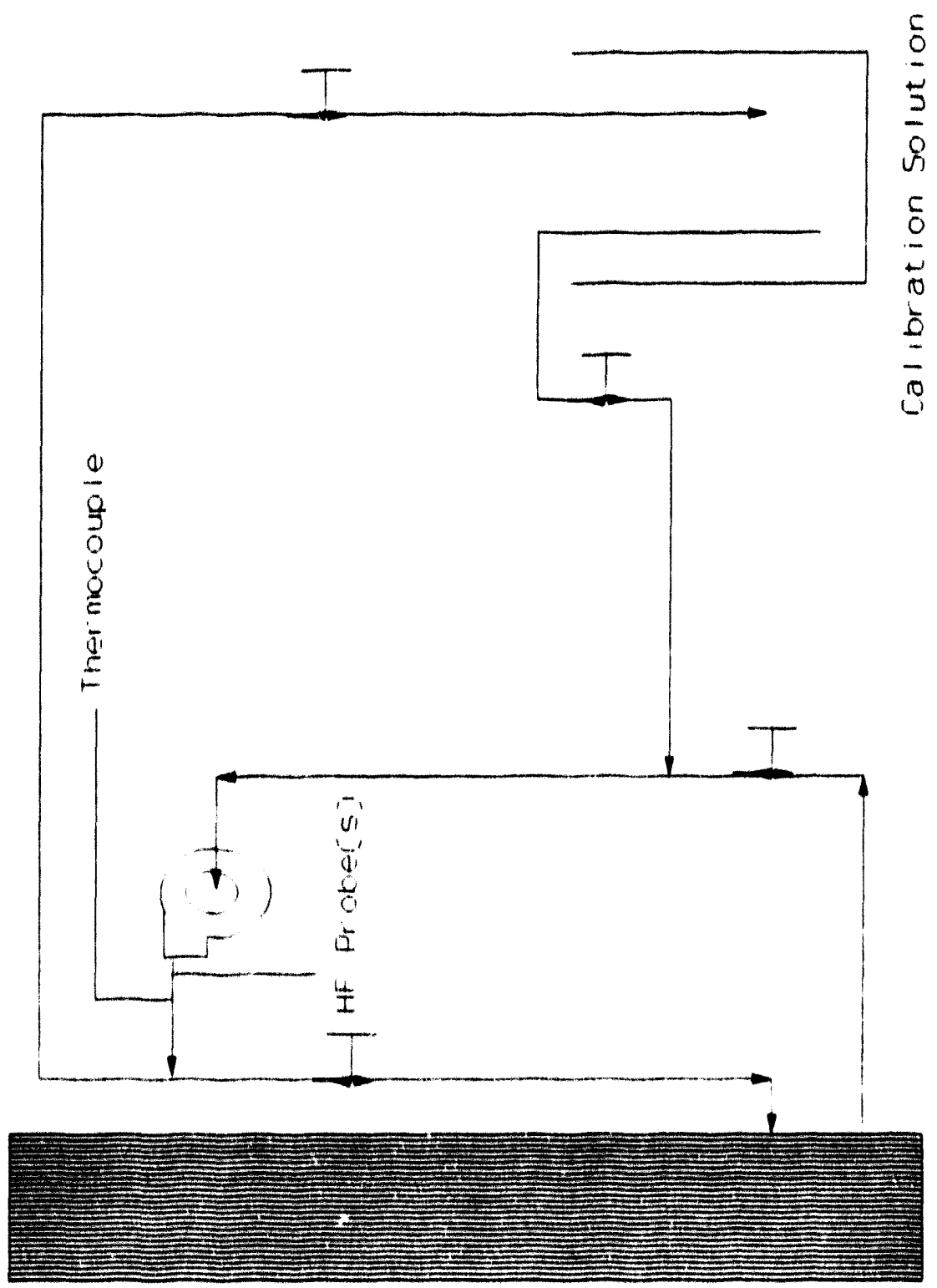

FIGURE 2.0.2 HP PROBE RECIRCULATION LOOP 


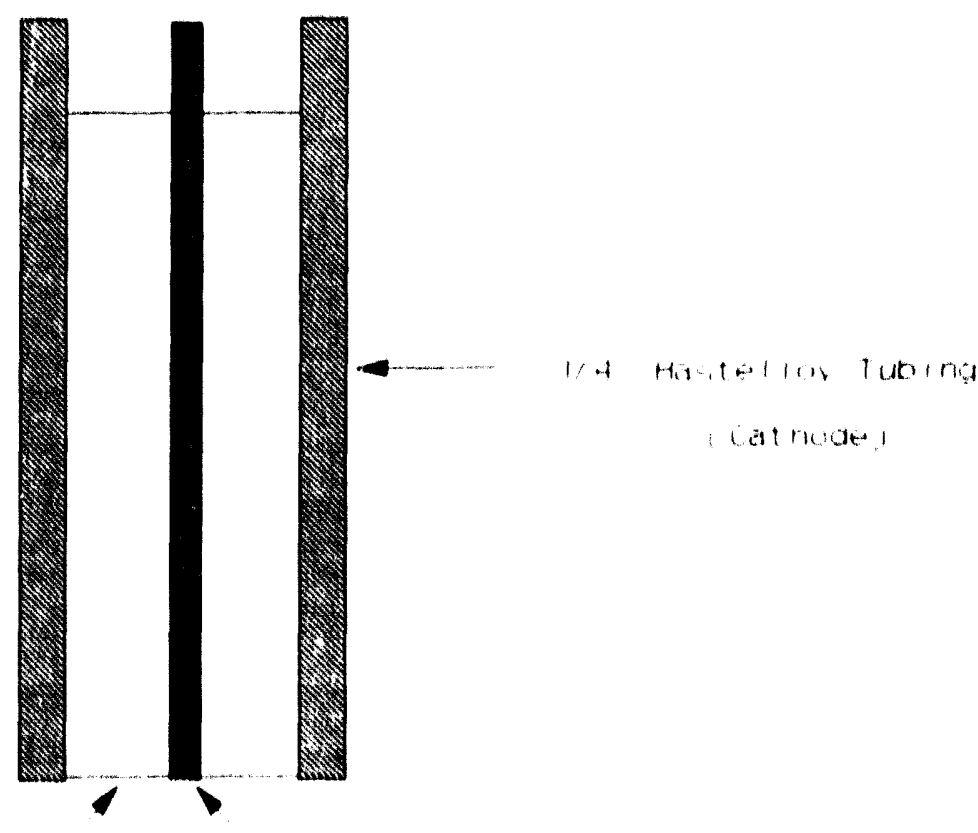

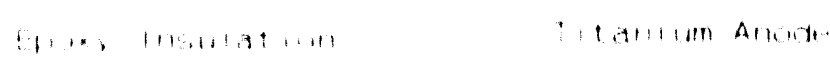

FIGURE 2.0-3 HF PROBE

The Fluorinel Pllot Plant (FPP) reagent tanks for water (FPP-VES-107), 13M nitric acid (FPP-VES-108), and 2.2M aluminum nitrate (ANN) (FPP-VES-109) were used as feed tanks for the complexing reagents used in the first addition. ANN for the second addition was obtained from a carboy. The complexing reagents were added to the system using peristaltic pumps which were feedback controlled using turbine flowmeters.

During the initial complexing step, water was added first, followed by a mixture of ANN and nitric acid. The use of a static mixer after each reagent addition ensured adequate mixing of the streams. After the initial addition of complexant, a series of free $H F$ measuring probes, in conjunction with thermocouples to compensate for temperature effects, followed the reaction. The information provided by the probes was used to determine the amount of ANN required at the first and second addition points. After the second addition was made, one more reading was taken downstream to determine if the product was adequately complexed. The final product was collected in a 770 liter 
stainless steel vessel equipped with density and level monitoring instrumentation.

\subsection{CONTROL SYSTEM}

\subsection{Current Status}

The In-line complexing control scheme is substantially complete. Individual reagent flow control loops are an essential feature of the process. This measurement is currently performed by turbine flowmeters in the mockup. This type of meter has an inherent bias with high viscosity fluids, which varies with rate. Currintly there is no compensation for this effect. Flowmeter characterization studies are planned which will allow the control system to correct the Figure 2.0-1 ILCM System flow signals. The plant may use another type of flowmeter. The control scheme would work with any accurate meter. The most recent test was performed at a $4 \mathrm{liter} / \mathrm{min}$ dissolver product feedrate. This required larger flowmeters and higher capacity pumps than previous runs, but the same control system gave good performance with the modified equipment. The current configuration uses 7 interacting PID control loops and an additional on-off loop for heating the feed. A setpoint track/run feature is used to provide bumpless startup of cascade loops (see Appendix A).

\subsection{Development History}

The MICROMAX process control system was selected to control in-line complexing. It was already installed for use with the Fluorinel Pllot Plant and GFD dissolver. It had analog 1/0, PIC loop control, and logic and calculational ability that made it a logical choice for in-line complexing. The original control system for in-line complexing was strictly feedforward. The first aluminum nitrate addition was based on equations used for vessel adjustment based on $[F],[Z r], S p G$ and temperature of the feed solution. A TI probe measured [HF] downstream of the first reagent addition point and calculated the additional ANN to be acded downstream. Additions of nitric acid and water are calculated by a running mass balance to meet product 
targets and were added near the first ANN location. This system gave acceptable results but required constant attention and frequent adjustment by a skilled chemist.

The development of In-line Complexing required precise flow control. Metering pumps are used for all feeds to the process. It was found that these pumps did not have linear output, so a characteristic response curve was generated for each pump and programmed into the MICROMAX. This improved pump response, but was not entirely satisfactory as the back pressure on the pump affected flow. kuns 1 and 2 were performed with this configuration.

A feedback loop was added to correct the initial ANN addition based on the probe response. At the same time orifice flowmeters were added to the ANN, nitric acid, and water additions. The flowmeters provided measured signals for closed loop control of these flows. It was especially important to be able to add measured amounts of nitric acid and water since the product concentrations could not be measured directly. The feed solution was too corrosive to measure with available orifice meters so its fiowrate was measured by level depletion. This gave an accurate value in theory, but was the difference of two noisy values over a short time base. Numerical damping gave some improvement, but the results were still marginal.

The ANN flow loop became the inner loop of the ANN control system. The outer loop was a combination of the feedforward calculated value and a feedback PID function operating on the probe signal. The feedforward contribution undercomplexed the feed solution and the feedback signal brought the free [HF] to the target setpoint. The piping arrangement between the addition point and the probes included a tube to allow residence time for the reaction to reach equilibrium. An adaptive tuning constant was selected to deal with the variable time lag in the outer, probe-controlled loop. The inner loop was the orifice meter-pump flow controller which received a rate setpoint from the outer 1oop.

The process performed well during run 3 with the controller correcting upsets. The time lag due to the solution holdup prior to the probe location resulted 
in slow oscillations which took about 30 minutes to dampen out. The final ANN addition system was operated in the same feedforward mode that was used before. This system did not add ANN except during upsets to the process because the first loop controlled to setpoint. The final ANN addition system could be considered a safety backup for this reason. The probe readings used to control the system had a bias that caused high readings so the final product was slightly overcomplexed (free $[H F]=0.040 \mathrm{M}$ vs a $0.045 \mathrm{M}$ target) during the first run with this control scheme, but still within acceptable limits. The control system is expected to work as well with unbiased probe readings. The primary weaknesses of the system at this stage of development were rate control by level depletion and zero bias on the orifice meters due to difficulty bleeding the meters.

In- 1 ine run ILCMOO4 demonstrated the feasibility of this system for Coprocessing with Aluminum dissolver product. A mixture of ANN and nitric acid was used as a complexing reagent. This required modification of the nitrate and ANN mass balance equations, and rewiring and relocation of other equipment. Run 4 succeeded in demonstrating the flexibility and adaptability of this process.

Later in the development work, turbine meters were added to measure all flows except final ANN to improve accuracy. A suitable material turbine meter was found for the feed solution, allowing closed loop feed control which was a great improvement over the level depletion method. The MICROMAX control logic was modified to accept the pulse frequency signals from the turbine meters. An additional control loop was added for heating the feed tank. Run 5 was performed with this configuration.

A new probe location was added to reduce the time lag in the probe loop, thereby improving the response of the controller. The new location gives about 30 seconds residence time at typical flows. This probe provides the signal for a third controller in the ANN cascade added between the other two. The new loop controls the flow loop and in turn is controlled by the original probe loop, making this a triple-effect cascade controller. If the reaction is incomplete at the new probe location, the outer loop trims the setpoint to 
compensate for $i t$. This improved response time by a factor of 4 during run 6 . A turbine flowmeter was also added to the final ANN flow during this run. It was configured as the cascaded inner loop of the final ANN demand. The primary control system worked so well that the demand for final ANN was infrequent and the rate was low. The flow loop was determined to be unnecessary and the configuration was returned to direct control of final ANN.

The most recent in-line complexing runs have been performed at the plant design feed flowrate of $4 \mathrm{liters} / \mathrm{min}$. Higher capacity flowmeters and extra pump heads for greater capactty were required for some reagents. Run 7 was performed with this configuration unt 11 terminated as the result of flowmeter plugging. After this, a filter was installed on the feed stream to prevent flowmeter plugging and run 8 was performed successfully.

The in-line complexing control system combines complex feedforward/feedback loop control with chemical equilibrium calculations, a feedforward correction calculation, mass balance equations, and a special probe reading converted to a free $[\mathrm{HF}]$ reading all in a real-time continuous process.

\subsection{RESULTS AND DISCUSSION}

\subsection{HF Target Attainment}

As discussed in the controls section, the method of HF target attainment gradually evolved with the development of the control system. While initial attempts entailed only feedforward control, the final system involved the use of feedforward/feedback cascaded control loops.

Figure 5.1-1 is a plot of the percent deviation of the final free HF concentration (which represents all of the solution gathered during an experiment) from its targeted value of 0.045 for all of the system runs. The impressive system performance during run 1 is deceptive in that the system was not self-regulating. During this run it was noted several times that the free $H F$ reading at tube 3 was too high (refer to Figure 2.0-1). As a result it was necessary to change the parameters used to calculate the ANN flow five times. 


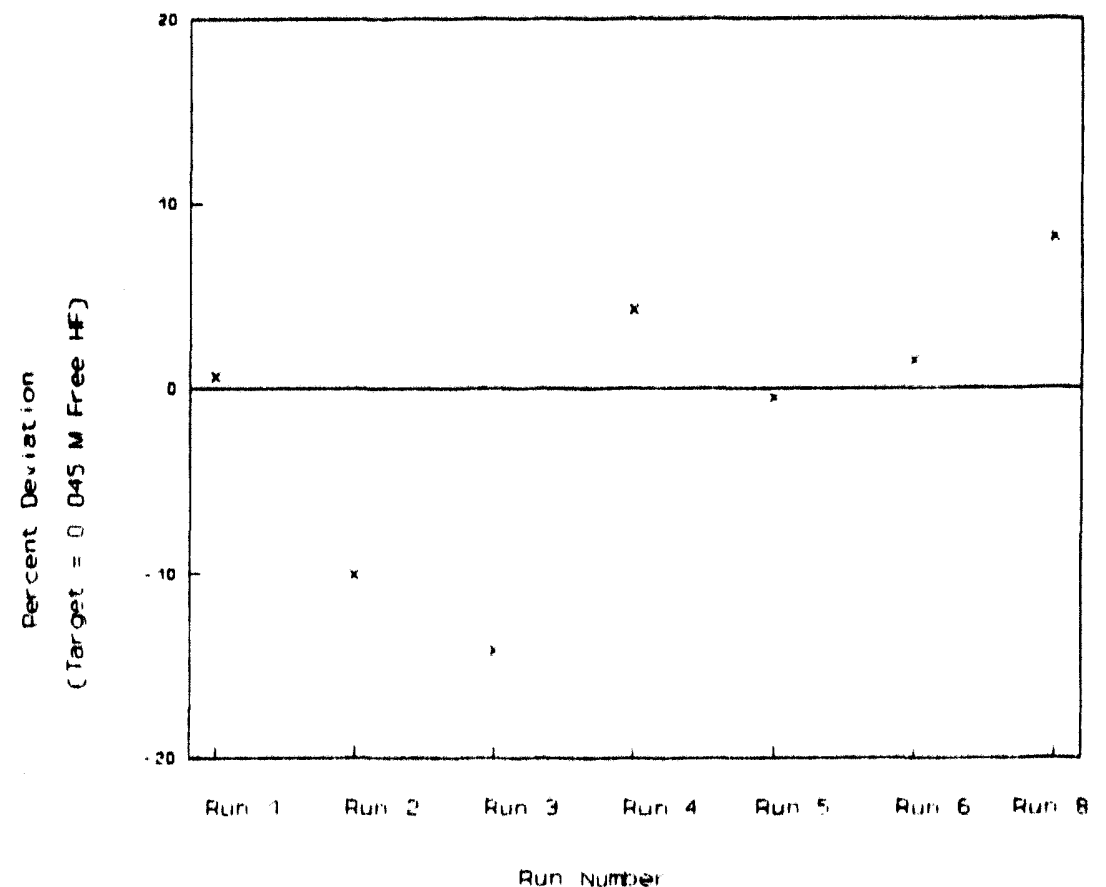

\section{FIGURE 5.1-1 PERCENT DEVIATION OF COMPOSITE FREE HF CONCENTRATION FOR ALL ILCM RUNS}

Thus, the system required constant operator intervention and it was merely coincidental that the deviations observed during the run averaged out to the proper overall concentration. The same control scheme was used during the second run with the goal that a better initial estimate of the HF concentration would allow the system to be self-regulating. The data point for run 2 in Figure 5.1-1 shows that the system's performance was worse than the first run.

At this point, it was apparent that a feedforward/feedback control scheme would be better suited to attaining the target without requiring operator intervention. In preparation for this run another recirculation loop was added at the top of the first column as is shown in Figure 2.0-1. The intended goal was to decrease the lag time between the ANN addition and the subsequent probe response. While the control scheme seemed to work as can be seen from Figure 5.1-2, the poor performance of the system with respect to 


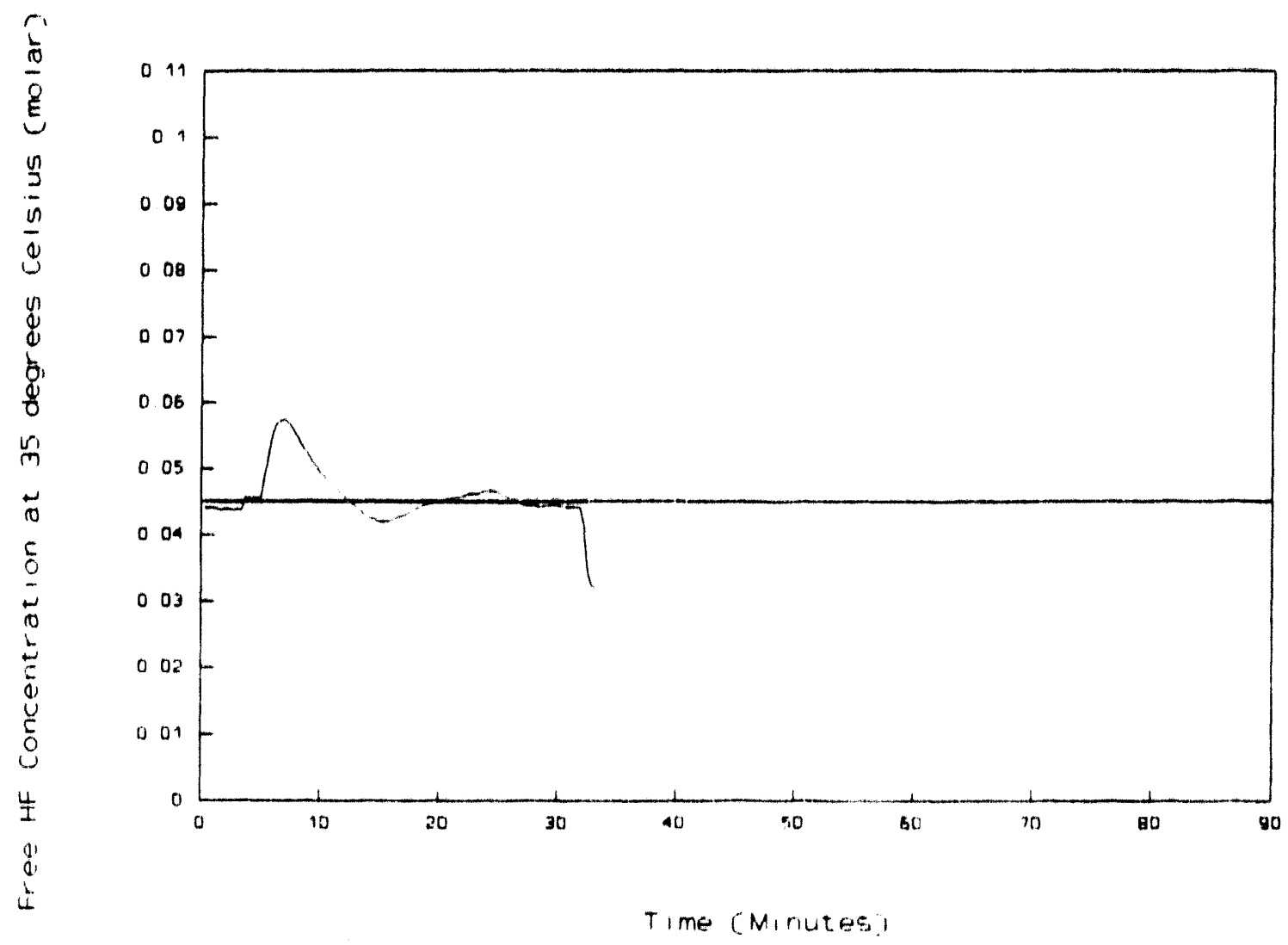

Figure 5.1-2 FREE HF READING AT TUBE 1 DURING RUN 3 VERSUS TIME

free HF concentration (shown in Figure 5.1-1) is attributed to improper calibration of the probe at the start of the run.

The same control scheme was used during run 4 , with the exception that a mixture of HNO3 and ANN was used in place of ANN to demonstrate the ability of the system to use the product from the aluminum dissolvers during coprocessing. This required changing the nitrate mass balance equations to account for the difference in nitrate concentration between ANN and the mixture used during this test. Although the test was a success from this standpoint, the initial estimate of the $\mathrm{HF} / \mathrm{Zr}$ ratio was too high, and resulted 
in the feedforward contribution providing more of the ANN/HNO3 mixture than was needed to complex the product solution to $0.045 \mathrm{M}$ free HF. At this time the control system had nut been set up for two-sided control, and although the feedback portion of the control scheme could compensate for too little complexant, it could not compensate for an excess of complexant. Thus, it was necessary to change the HF/Zr ratio several times in order to achieve the 0.045 setpoint.

Prior to Run 5, the control system was changed to allow for two-sided control (see discussion in APPENDIX A). Figure 5.1-3 shows the damped approach to set point which resulted from the control scheme after the initial start-up disturbance. In addition, this Figure shows that the peaks and valleys resulting from this control scheme roughly compensate for each other, resulting in an average product composition which was close to the desired value as is shown in figure $5.1-1$.

In preparation for run 6 , the system was modified by placing a recycle loop closer to the addition point for ANN as shown in Figure 2.0-1. It can be seen from Figure 5.1.4 that the system modifications resulted in a significant decrease in both the period of oscillation and the decay ratio. In addition, the final product was close to the desired value as is shown in figure 5.1-1.

During run 7 an attempt was made to operate the system at the design basis feed flow rate of $3.51 \mathrm{pm}$. Problems were encountered during run 7 with solids plugging the ZrDP meters and the run had to be aborted'. However, the addition of a filter enabled the system to be successfully operated at the design basis flowrate during run 8 . The fact that the final HF concentration shown in Figure 5.1-1 is above the targeted value is attributed to the system upsets during run 7 resulting in undercomplexed 2rDP going to the final collection vessel (the solutions from runs 7 and 8 were collected in the same batch.)

It can be seen from Figure 5.1-5 that further improvement in system control was realized through the decrease in lag time which resulted from operating at 


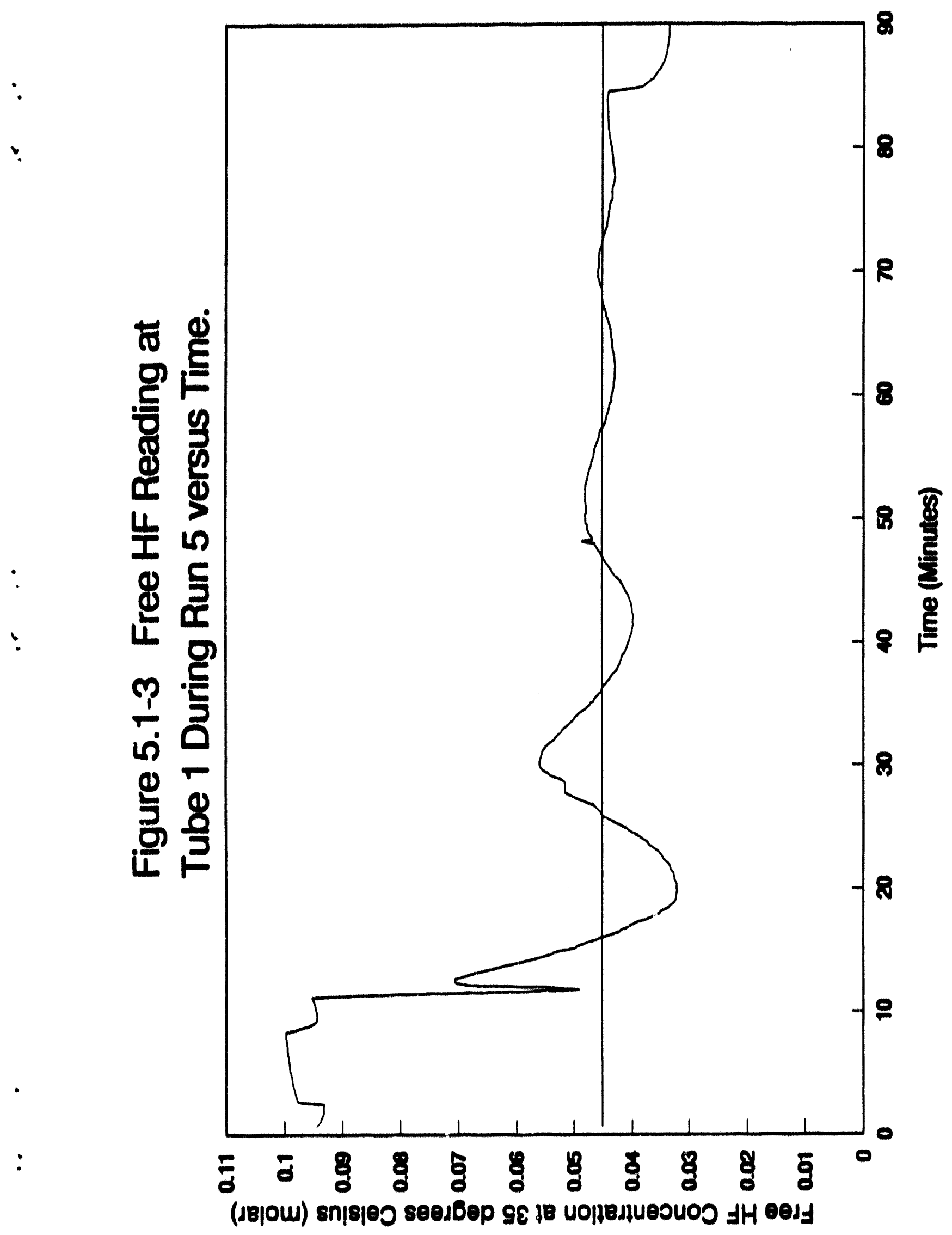




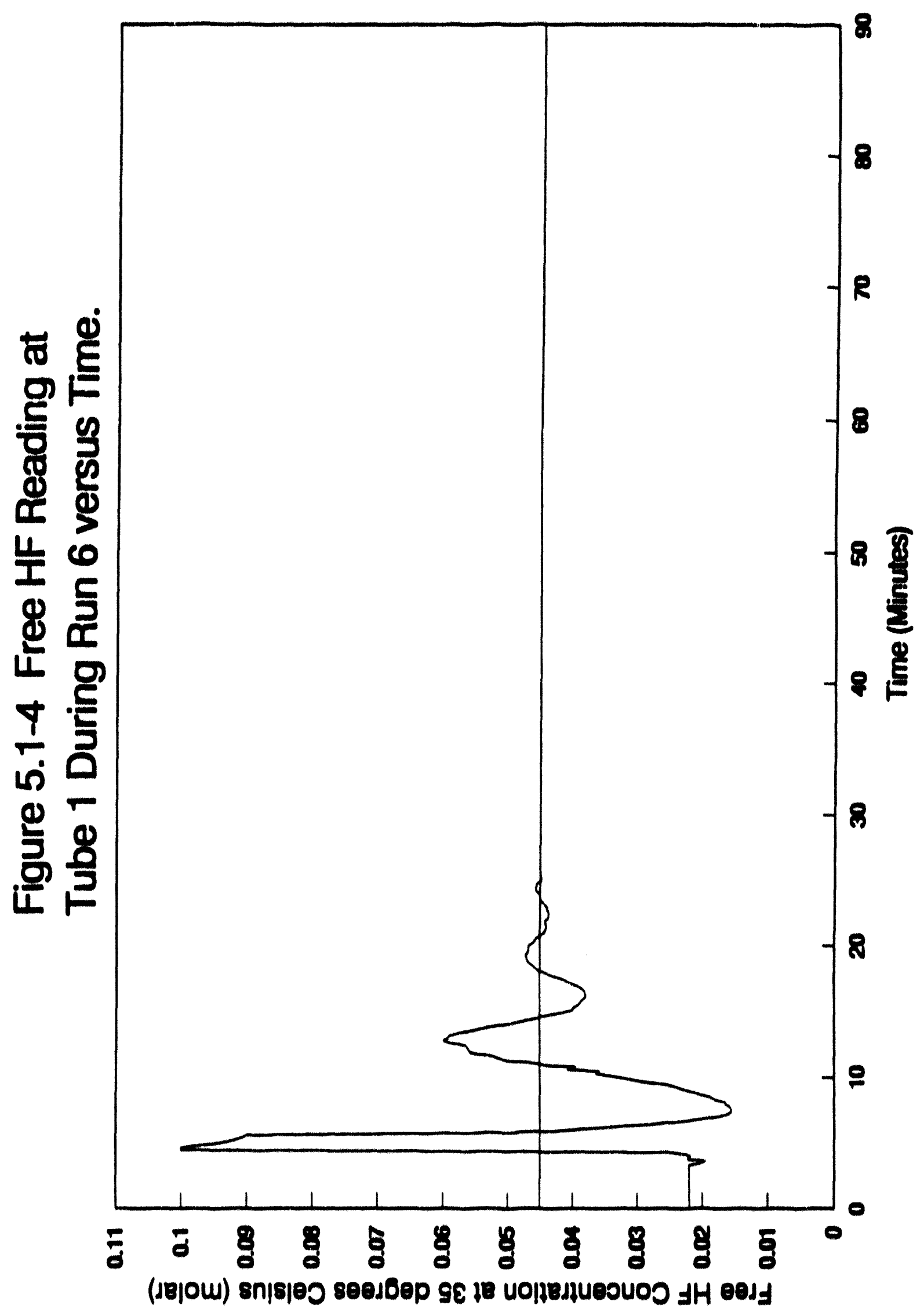




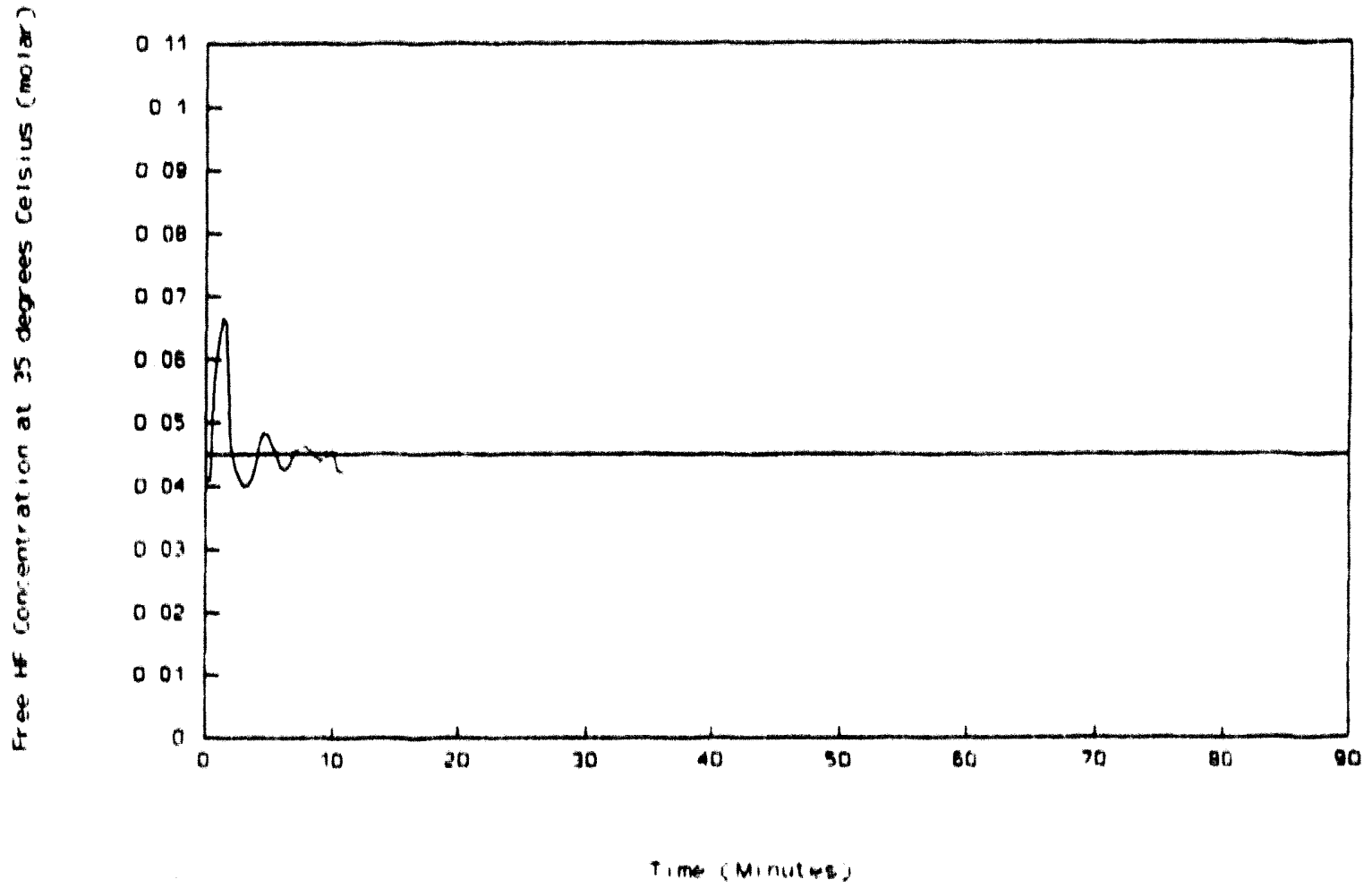

FIGURE 5.1-5 FREE HF RFADING AT TUBE I DURING RUN 8 VERSUS TIME

the higher flow rate. This result indicates that system control at lower flow rates could be improved by moving the first recirculation loop further upstream. A summary of the periods of oscillation and decay ratios for runs 5, 6, and 8 is contained in Table 5.1-1.

In addition to obtaining information on the performance of the control system during runs 6 and 8, the apparent residence time for the complexing reaction was also bracketed. This was accomplished by comparing the probe reading at the first recirculation loop (at the bottom of column 1) to the reading at the second recirculation loop (at the top of column 1). A lower reading at the top of the column would imply that the solution did not have enough time to react before it reached the first loop and was consequently being overcomplexed. A comparison of the data for these two probe locations indicated that they were in close agreement during run $6^{2}$, however there was a consistently low blas in the reading at loop 2 relative to loop 1 during run $8^{3}$. (Loop 3 was in close agreement with loop 2 indicating that this was not 
TABLE $8.1-1$

Summary of Decay Ratios and Perlods of Oscillation for Runs 5,6 , and 8 .

Run Number

5

6
Period of Oscillation (ninutes)

18

7

3.5
Decay Ratio

0.8

0.6

0.3

simply an erroneous probe reading.) The est imated reaction residence time at the flow rates observed during run 6 was approximately 40 seconds (This value differs from that given in DSH-03-91 due to the fallure to account for the 10 seconds residence time in the l" line leading up to the first 4" tube.) The estimated residence time at the flow rates observed during run 8 was approximately 17 seconds. Thus, It appears that between 17 and 40 seconds were required for the complexing reaction to attain equilibrium under the observed experimental conditions. However, it is important to note that the temperature at column 1 was 41 degrees celsius during run 6 , whereas it was closer to 28 degrees Celsius during run 8 . While it is probable that the reaction will be slower at the lower temperacure, this effect has not been quantified.

\subsection{Probe Operation and Rellability}

While figures 5.1-3 through 5.1.5 indicate that the control system will control to set point, there is still uncertainty associated with the probe's ability to provide an accurate reading. Probe signals have been observed to drift apart by as much as $11 \%$ during system runs and complete probe fallures have also been noted". Subsequent observation of the probes indicated that the epoxy used to insure one-dimensional attack on the $i 1$ anode was falling from exposure to the process solution. While this is one of the more probable 
modes of probe fallure, fundamental understanding of the reaction mechantsm and mass transfer phenomena associated with probe operation is still lacking. This information should be obtained prior to bullding the process around the probe. (A collaborative research project with Montana State University for this purpose had been initfated at the time this report was being written.) Figure 5.2.1 compares the computer programb calculated free HF concentrations based on the RAL's analysis for 30 samples obtained during runs of the system to the probe readings. Although the significant scatter has been attributed to error in the RAL's analysiso, this plot indicates that the program may not be rellable for independently confirming the probe readings.

An unexpected and interesting result was observed during the fifth run of the ILCM. It was noted that a probe was not responding to the callbration solution during the beginning of the run so it was disconnected. Later inspection of the probe indicated that the probe had corroded back into the epoxy approximately 1/8th of an inch. This was estimated to be between one and two orders of magnitude greater than the corrosion observed for the other probes which had remained connected to the blas box during the experiment.

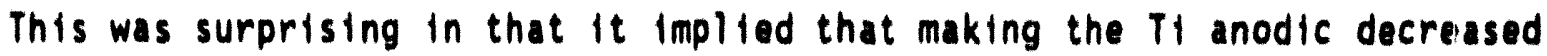
rather than increased its corrosion rate. It was suspected that this was the result of a change in the dissolution mechanism upon applying the blasi voltage to the $T 1$. A literature search revealed that 11 forms a passive oxida film upon the application of an anodic bias voltage in HF/HNO3 solutions'. This is attributed to a passivation reaction during which a dilute phase of adsorbed isolated metal hydroxide cons is transformed into a continuous oxide layer upon the application of sufficient potential". The $1102 \mathrm{f} 11 \mathrm{~m}$ is destroyed when the oxygen atoms (double bonding) are replaced by fluorine atoms (single bonding) destroying the 2-dimensional network protecting the metal ${ }^{\circ}$. The reaction by which this is thought to occur is given below ${ }^{10}$ :

$\mathrm{THO}_{2}+\mathrm{nHF} \cdots\left(\mathrm{THF}_{n}\right)^{\mathrm{nat}}+(n-4) \mathrm{H}^{*}+2 \mathrm{H}_{2} \mathrm{O}$

Observation of the probes after exposure to process solution under an optical microscope indicated that the metal was pitted. Thus, it is thought that the 
current produced by the HF probe results from HF attack on the $T 102$ f1Im and its subsequent regeneration. It is also possible that direct attack on the $T 1$ metal occurs if the $T 102$ flim is completely removed. In addition, it is likely that the formation of this oxide film accounts for part of the current splke of current observed during probe callbration".

Unexpected probe behavior was al so observed during a recirculation test intended to determine the long term rellability of the probes. Approximately 34 hours into the test the velocity of the solution past the probes was changed from $17 \mathrm{~cm} / \mathrm{s}$ (Reynolds number $=940$ ) to $11 \mathrm{~cm} / \mathrm{s}$ (Reynolds number = 590). Surprisingly, this $38 \%$ decrease in fluid velocity resulted in no decrease in the probe readings. The fact that there was no perceptible change in probe reading desplte change in the rate at which reactant was being supplied indicates that another process could have been limiting the response of the probe. It is possible that this could be either the reaction of $H F$ at the probe's surface or the formation of a film of corrosion products generating another layer through which the HF must diffuse prior to reacting with the surface. If the system is 1 imited by the reaction there are no implications related to probe operation. However, if the system becomes limited by HF diffusio due to accumulation of corrosion products or other material on the surface of the probe, this would increase the response time and cause a steady downward orift in the probe reading. In fact, steady downward drifts in probe reading have been observed during recirculation tests (although this may be attributable to epoxy swelling.) One method which could be used to determine if the accumulation of material on the probe is influencing the probe response would be to expose the probe to spikes or step changes in concentration at different times during recirculation tests. If the probe is limited by the reaction the response time will not be a function of the time of exposure to the process solution, whereas if a fllm of material is forming as time passes which limits the reaction, the response time will be a function of exposure time.

Due to the advantages of using metal Tees instead of plastic Tees for probe placement in the plant, a comparison etween the signal produced by probes 
1

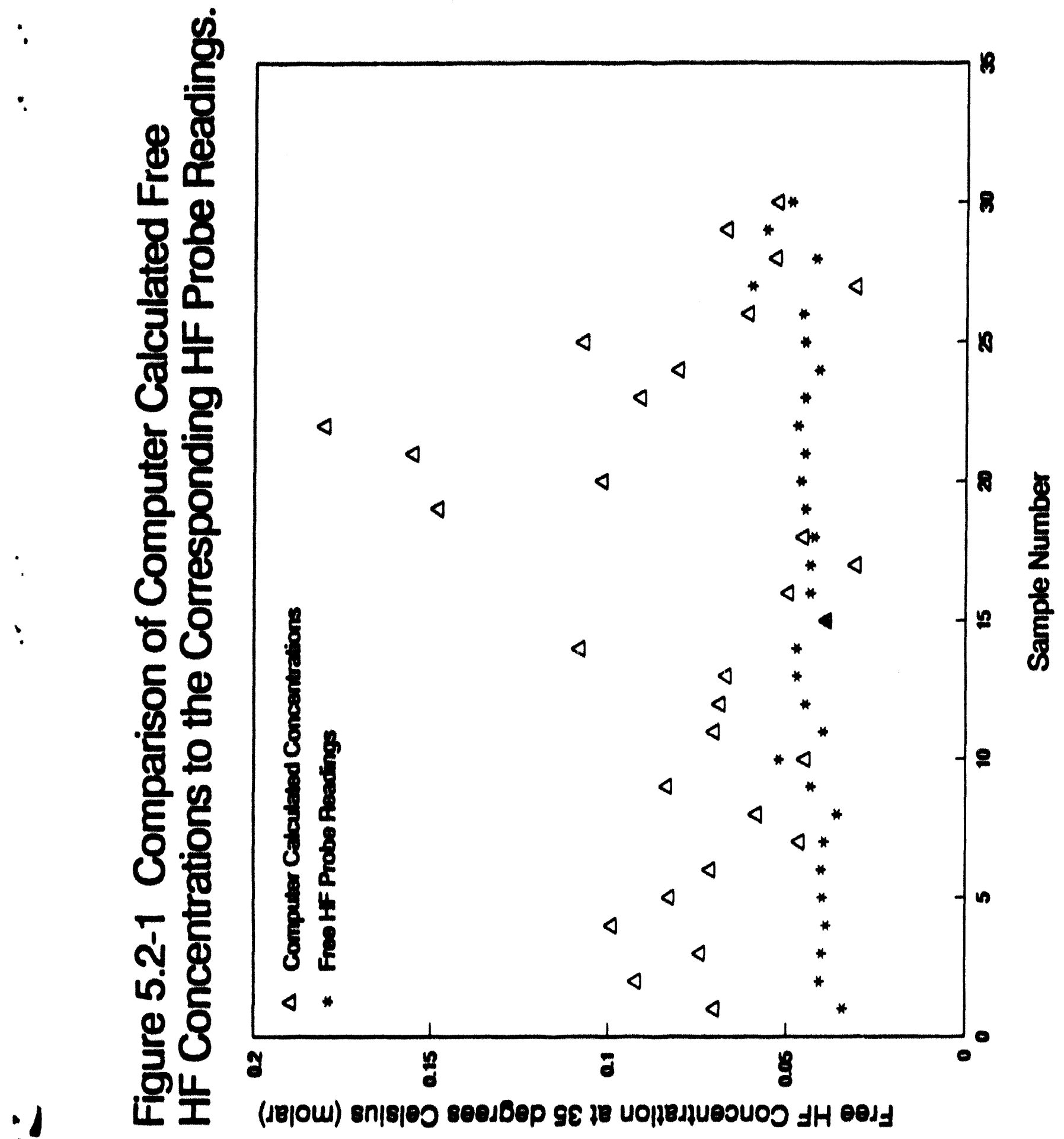


placed in adjacent stainless and Kynar Tees was made during run 8 . Prior to this run there had been an unsubstantiated opinion that the probes would not work if placed in a metal Tee. Placing a probe in a metal Tee grounds it, and the original probes had always been placed in plastic Tees to avoid grounding. However, due to the insignificant resistance to current flow in metals and the small amounts of current being generated, placing a probe in a metal Tee should merely serve to increase its effective cathode area. If changing cathode area influenced the operation of the probes then the rate determining step in HF determination would not be HF consumption at the anode, but hydrogen production at the cathode. This mode of probe operation would be unacceptable for a probe used to determine HF concentrations. Fortunately, the test results indicated that there was no difference in the readings produced by the two probes ${ }^{12}$. Thus, it appears that metal Tees may be used for probe placement in the plant. However, additional tests are recommended to confirm this result and a fundamental understanding of grounded versus ungrounded probe operation should be developed.

\section{3 $\mathrm{Zr}$ Target Attainment}

As discussed in the controls section, the $Z r$ target was attained with feedforward control using a simple mass balance. The system's deviation from this target was primarily the result of error in the determination of the reagent addition rates, and error in the equation used to estimate the amount of $2 r$ in the ZrOP feed. This equation is a function of density, and although the error in the density determination is only $\pm 1.4 \%{ }^{13}$, the equation increases the error to $\pm 8 \%$. This error combined with the error in the RAL's analysis (shown in Table 2.0.1) implies that a difference as large as $\pm 17 \%$ between the mass balance values and those provided by the RAL would be within experimental error (Al though the $95 \%$ confidence level is $\pm 13 \%$ ).

Figure 5.3-1 is a plot of the percent deviation of the final $2 r$ concentration from its target value based on the RAL's analysis of the final product along with the corresponding error observed for the nitric, water, 2rDP, and total ANN flows. Figure 5.3.2 compares the error based on the RAL's analysis to the 
error in $\mathrm{Zr}$ concentration which would be expected based on tank depletion and the $\mathrm{Zr}$ concentration in the recycle vessel from the density equation. It can be seen from Figure 5.3-2 that the mass balance values consistently agree with those obtained from the RAL's analysis within the error associated with the density equation and the analysis (i.e. $\pm 13 \%$ ). This supports the assumption that the tank depletion readings were accurate.

Referring to Figure 5.3-1, the relatively large deviations in the flow rates observed for runs 1 and 2 are attributable to operating the pumps without feedback control. The problem with attempting to control the system's peristaltic pumps without flowmeters was that they were subject to pressure effects (both inlet and outlet), viscosity effects, tubing hysteresis, and nonlinear response to control output. This nonlinearity produced the increase in error observed for the ZrDP pump during run 2 since it was run at a lower flowrate than in run 1. As can be seen from the Figure for these two runs, the resulting combination of higher than expected flows for water, ANN, and HNO3, along with lower than expected flow for the $\mathrm{ZrDP}$ resulted in $\mathrm{Zr}$ concentrations which were lower than the targeted values.

As a solution to these problems during run 3 , orifice flowmeters were placed on all of the pumps with the exception of the ZrDP pump (due to materials problems) and the ANNII pump (due to its relatively low flow rate.) In addition, the ZrDP rate was determined by observing the tank depletion rate and setting the control output to the pump at a value which produced the desired flow. As shown in Figure 5.3-1, this resulted in a considerable improvement in the system's overall performance.

One of the purposes of run 4 was to demonstrate the viability of coprocessing with the ILCM. In order to accomplish this, a mixture of nitric acid and ANN which was nitrate deficient was used in the place of ANN. Although from this standpoint the test was a success, the failure of the orifice meter which was used to meter this mixture resulted in a considerable amount of dilution and a consequent low concentration of $\mathrm{Zr}$ as can be seen from Figure 5.3-1. 


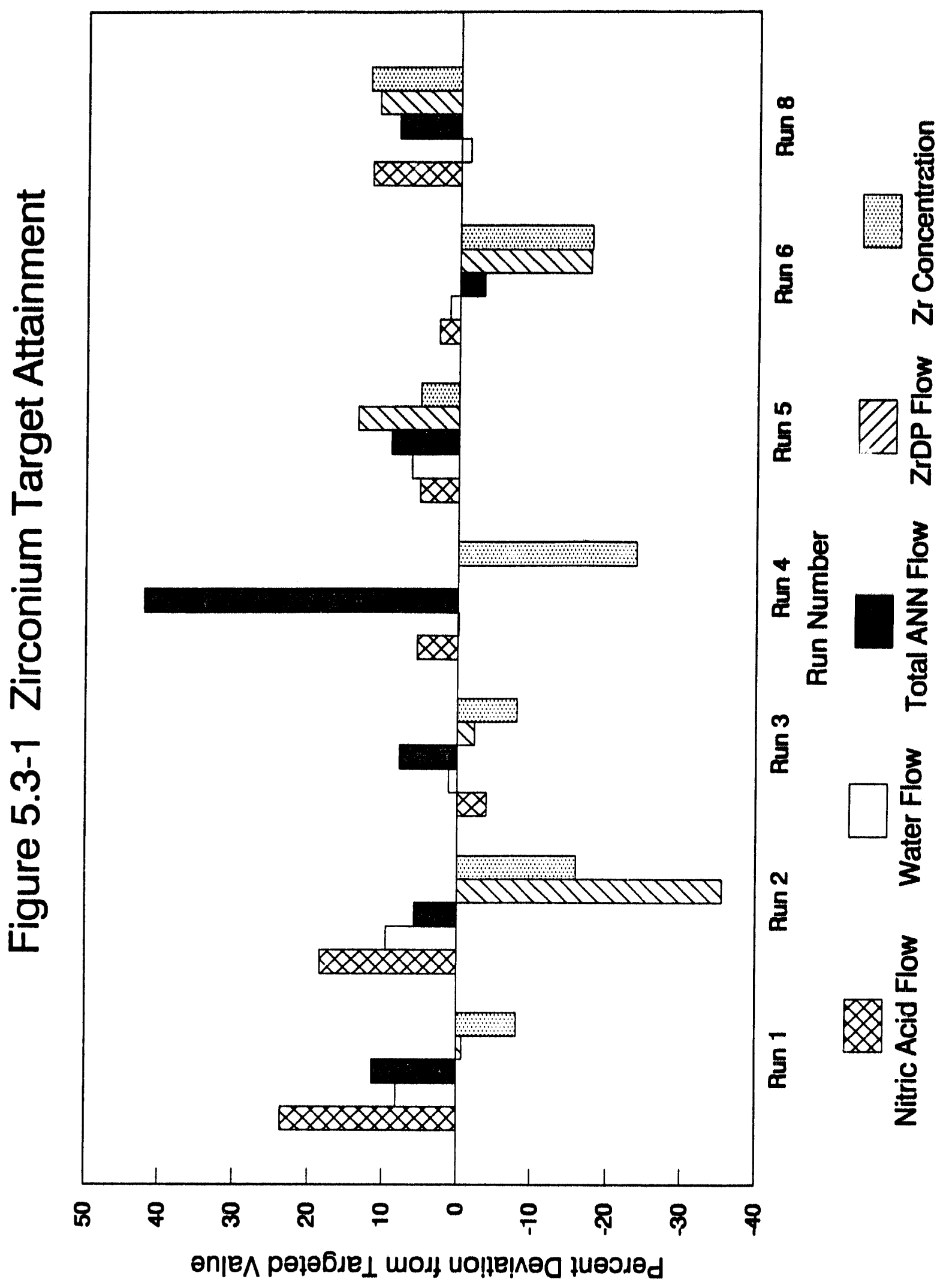




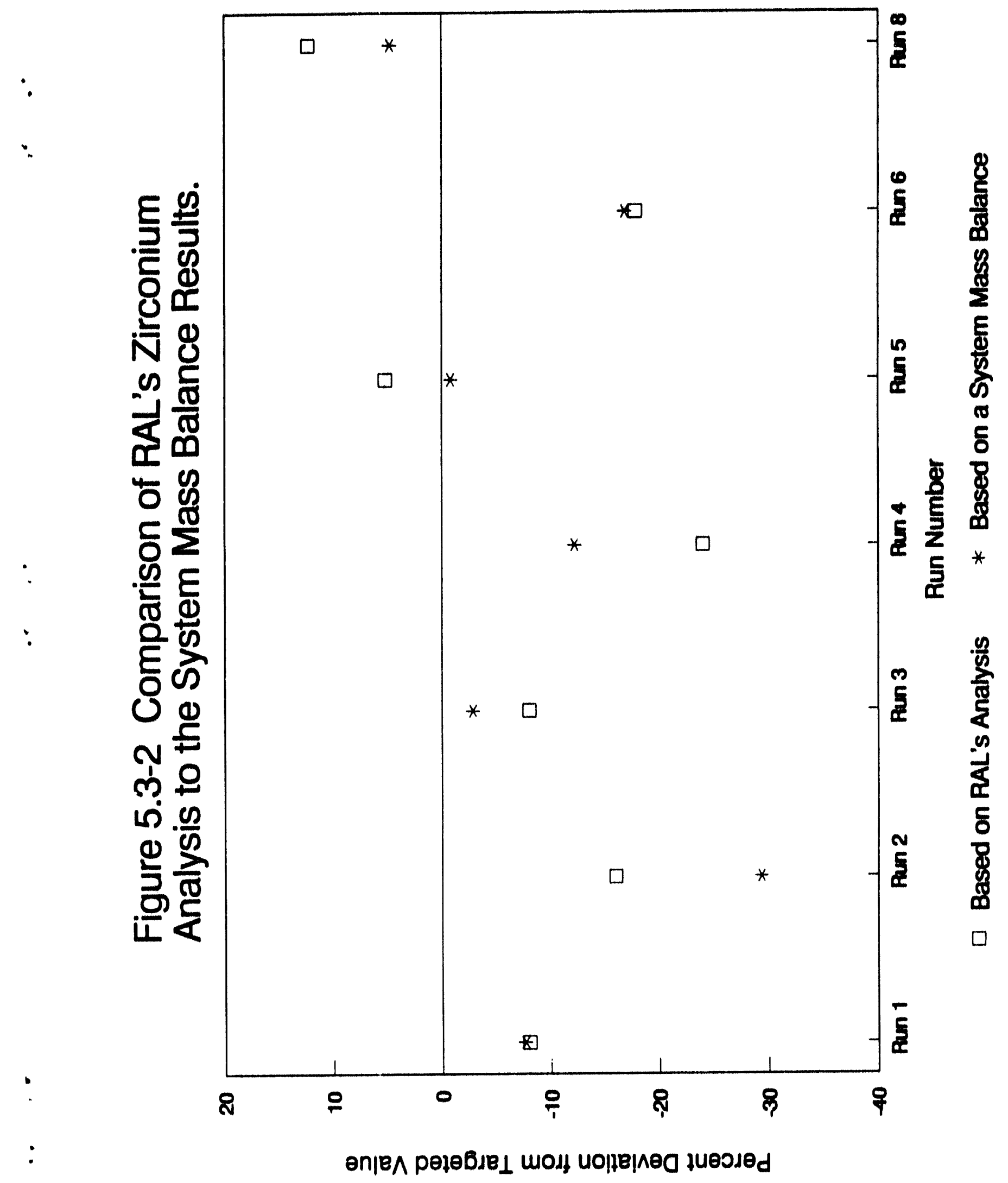


After this test all of the pumps (with the exception of the ANNII pump) were converted to feedback control using turbine flow meters for run 5 . Due to the hazards associated with calibriting the meters with reagents such as 2 rDP, and HNO3, it was assumed that the flowmeters would exhibit a linear relationship between the pulses produced by the turbines and flowrate. Although this is not always the case ${ }^{14}$, it proved to be an adequate first approximation as can be seen from the results for run 5 in figure 5.3-1

The relatively poor performance with respect to the $2 r$ target which was observed during run 6 is attributable to the 20 percent bias in the 2rDP pump. It is suspected that this bias resulted from solids plugging the screen in front of the flowmeter producing cavitation ${ }^{16}$. This in turn caused the meter to read a higher flow rate than was actually occurring resulting in less $\mathrm{Zr}$ in the final product.

In preparation for run 7, two flowmeters were placed into the ZrDP feed line to prevent an abort of the run due to a single flow meter being jammed by solids. Despite this precaution, the filters on both meters plugged and the run was aborted. The filters were subsequently disassembled and were found to contain a rubbery substance. It is suspected that this material came from thread sealant which was used on the recycle vessel.

Prior to run 8 a new filter was placed in front of the ZrDP flowmeter and this solved the plugging problem. In addition, larger flowmeters were placed on the ANN and HNO3 pumps to enable the system to operate at the design basis flowrate. The error in the $2 r$ concentration during this run was primarily the result of a higher than expected flow of ZrDP. This probably resulted from calibrating the meter with water prior to this run and the higher viscosity of the 2 rDP resulting in a bias in the flowmeter reading. It is suspected that the same phenomena is responsible for this high bias in the flow from the ANN and HNO3 pumps. 


\subsection{Nitrate Target Attainment}

As with the $2 r$ target, the nitrate target was attained with feedforward control using a simple mass balance. The error observed in the system's attainment of this target was primarily the result of errors in the determination of the amount of nitric acid and ANN being added.

Figure 5.4-1 is a plot of the percent deviation of the final nitrate concentration from its target value based on the RAL's analysis of the final product along with the corresponding errors for the nitric, water, ZrDP, and total ANN flows. Figure 5.4-2 compares the deviation based on the RAL's analysis to the nitrate concentration which would be expected based on tank depletion and the nitrate concentration in the ANN and HNO3. Figure 5.4-2 shows that the mass balance values agree (with the exception of run 4 which will be discussed later) with those obtained from the RAL's analysis within the assoctated analysis error. This supports the assumption that the tank depletion readings were accurate.

It can be seen from Figure 5.4-1 that higher than expected flows of HNO3 and ANN during run 1 resulted in the nitrate concentration exceed the target value. The same can be said for run 2, al though the lower than expected flow of 2 rDP resulted in an increase in the relative error. During run 3, a slightly lower than expected flow of HNO3 resulted in the iftrate being lower than its target.

Although the results for runs $1-3$ are easily explained, the results for run 4 are not. Referring to Figure 5.4-2, it can be seen that the nitrate from the RAL's analysis does not agree with the value resulting from a mass balance. Evidence indicates that the error can be attributed to the RAL's analysis of the final product. The first reason for this assessment is that the low concentration of $2 r$ in the final product is consistent with fallure of the ANN/HNO3 mixture's flowmeter and consequent dilution of the final product. Secondly, a mass balance was performed on the Al in the system and this indicated that the analysis of the HNOB/ANN mixture used during this run was 
reasonably accurate. Finally, comparison of the nitrate predicted to be in the final product by the following expression:

$$
\text { NO3 }=\text { HNO3 +3AI }
$$

Where:

HNO3 - Acid - $(F-4 Z r)^{10}$

to that given by the RAL's analysis for this run indicated that the analysis values were lower then would be expected based on this equation (refer to the data point shown for run 4 in Figure 5.4-2)

The ZrDP feed for runs 5, 6, and 8 was all produced with minor quantities of HNO3 as an oxidant during the dissolution process. The slightly high bias in the mass balance values shown in Figure 5.4-2 for runs 5 and 8 is attributable to the erroneous assumption that all of this nitrate was consumed. The same can be said for run 6, al though the low ZrDP flow was the primary contributor to the error.

\subsection{Corrosion}

Due to the short duration of coupon exposure, the data obtained on the corrosive effects of HNO3/HF mixing in the system is inconclusive. The apparent coupon weight loss was within the error of the measurement.

\subsection{RECOMMENDATIONS FOR FUTURE WORK}

Based on the results and problems encountered during operation of the ILCM, future work to ensure the successful operation of the integrated pllot plant and the full scale factlity is recommended.

First, a robust probe should be developed which will operate reliably under the process conditions expected in the plant. While the performance of the current probe was found to be acceptable during relatively short mockup runs, occasional failures and unexplained drifts indicate that it is inadequate for 


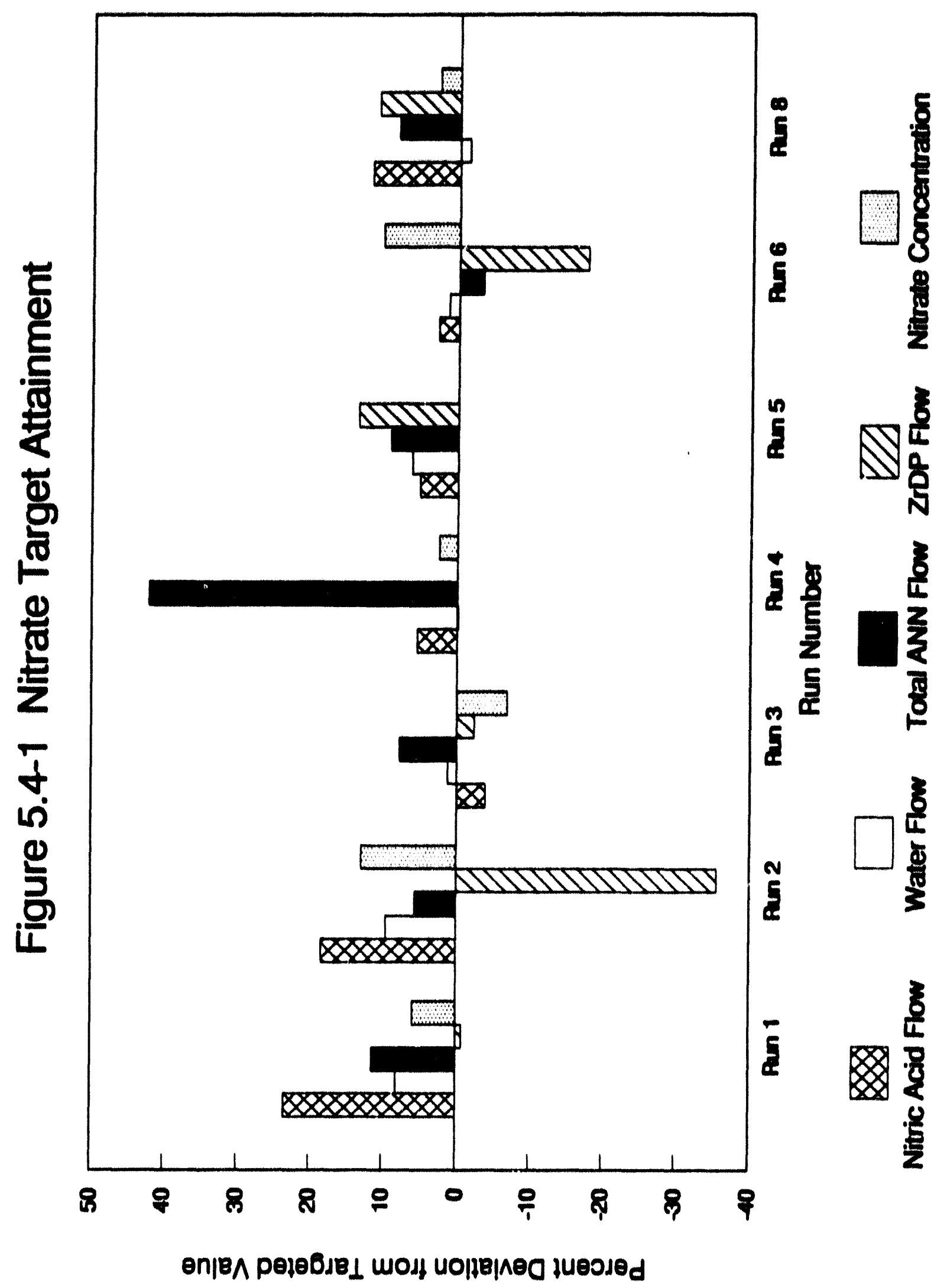




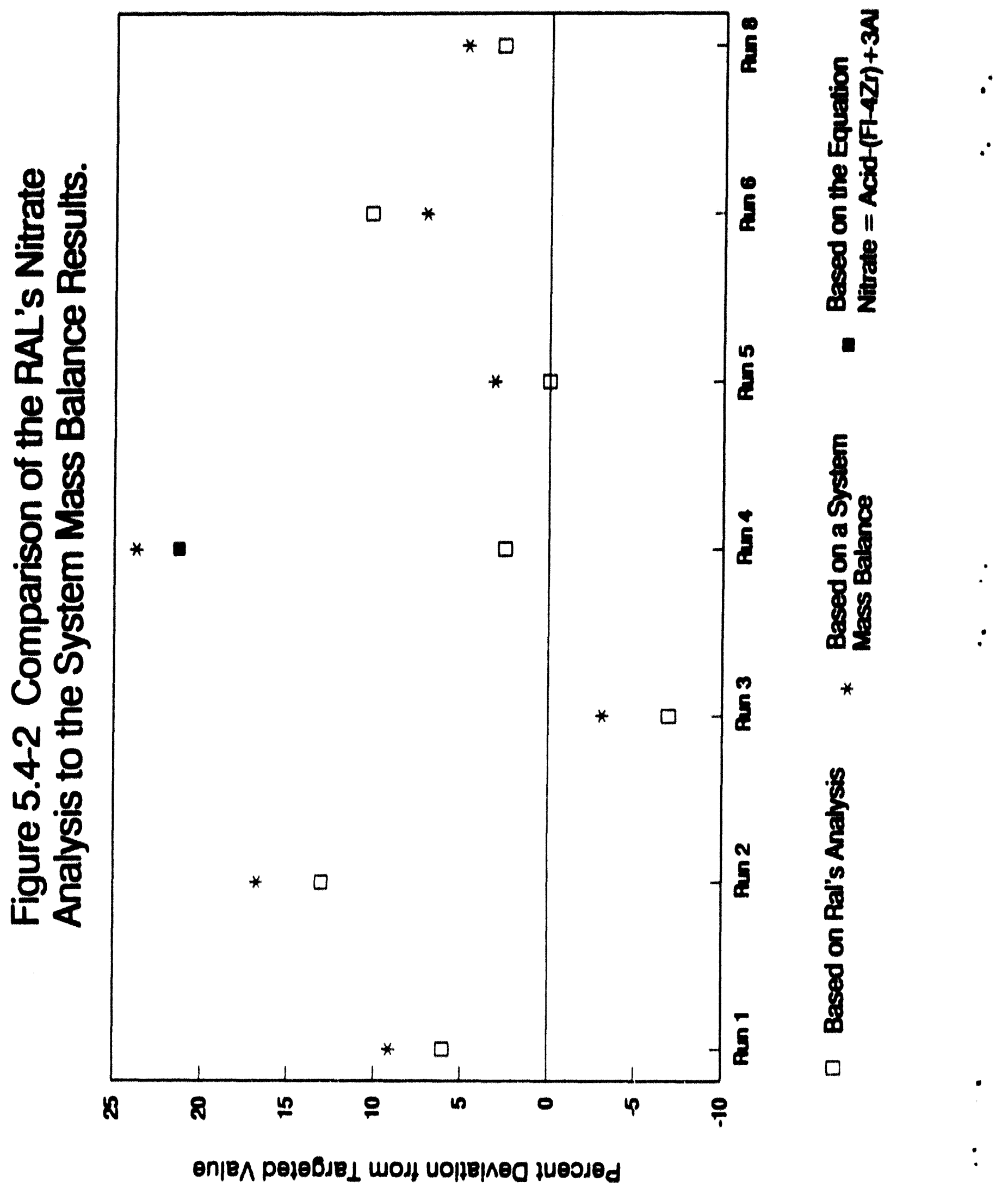


long term plant service. In consideration of the important role the HF probe would play in the full scale facllity, a fundamental understanding of the reactions and transport phenomena associated with its operation should be developed.

Additional work will also be required to determine the types of equipment needed to provide the necessary control of reagent flows in the plant. Although pumps and flowmeters would be acceptable for the cold reagents, they would be unacceptable for radioactive service such as 2rOp flow determination. One posstbility for measuring the ZrDP flow and its specific gravity would be a corriolis mass flowmeter. Although vendor data indicate this meter is capable of providing the required accuracy with respect to flow rate and specific gravity, it has a significant pressure drop assoclated with it. Another possiblitty would be a metering head pot, however, actual tests with this equipment should be performed to ensure it can provide the required accuracy.

Experiments investigating the corrosion effects of HNO3/HF $m i x i n g$ should be set up. Inttially, this should involve the use of limiting conditions, 1.e. HNO3/HF without ANN present in the solution. If this test indicates that further investigation is warranted, then a continuous flow experiment should be setup on a reduced scale to avold generating excessive amounts of product solution.

Additional pilot plant runs should be carried out to confirm the required residence time for reaction under all anticipated operating conditions. During these runs the possiblitty that smoothing the incoming HF probe signal coupled with derivative control could improve the system's abllity to deal with disturbances should be investigated. In addition, the magnitude of the start-up disturbance could be reduced via closer tuning of the feedforward contribution. 


\subsection{CONCLUSIONS}

In-line Complexing is a viable concept with the system consistently approaching the HF (error range $-14 \%$ to $8 x), 2 r(-16$ to $12 x)$, and nitrate targots $(-7$ to $13 \%)$.

The necessary residence time for the reaction under the conditions observed during these experiments appeared to be between 0.3 and 0.67 minutes.

The tests indicated that two points for ANN addition are adequate. During the last five runs of the system complexing was essentially complete through the use of the first ANN addition point only. However, the second addition system may be required in the event of extreme upset conditions or fatlure of the first system.

A test of the system carried out with a mixture of ANN and nitric acid demonstrated the applicabllity of the process to co-processing.

Metal fittings appear to be acceptable for placing probes in the plant, al though further testing and theoretical studies are recommended.

Concerns about the rellability of the HF probe need to be addressed. In addition, a fundamental understanding of its operation should be developed. 


\section{REFERENCES}

1. Halverson, D.S., "Summary of In.Line Complexing Mockup Run ILCMOOI and ILCMOO8," DSH-05.91 (June 27, 1991).

2. Halverson, O.S., "Summary of In.Line Complexing Mockup Run ILCM006," DSH.03.91 (March 27, 1991).

3. Halverson, D.S., "Summary of In-Line Complexing Mockup Run ILCMOOI and ILCMOO8," DSH.05.91 (June 27, 1991).

4. Halverson, D.S., "Summary of In-Line Complexing Mockup Run ILCMOOS," DSH-10.90 (December 10, 1990).

5. Personal communtcation James A. Murphy $2 / 10 / 90$.

6. IbId.

7. Sutter, E.M.M., Goetz-Grandmont, G.J., Corrosion Science, Vol 30, D. 463 (1990).

8. Sato, N., corrosion science, Vol, 31, P. 3 (1990).

9. Ibid D. 14.

10. Sutter, E.M.M., Goetz-Grandmont, G.J., Corrosion Science, Vol 30, D. 466 (1990).

11. Sato, N., Corrosion Science, Vol, 31, D. 6 (1990).

12. Halverson, D.S., "Summary of In-Line Complexing Mockup Run ILCMOOI and ILCMOO8," DSH.05.91 (June 27, 1991). 
13. "Generic Measurement Principles and Error Sources," Draft Letter L.D. Watson to L.E. Soderquist; May 1, 1985.

14. OMEGA Flow and Level Handbook, pg. F-3, F-4; 1989.

15. Halverson, O.S., "Summary of In-Line Complexing Mockup Run ILCMOOI and ILCMOOB," DSH-05.91 (June 27, 1991).

16. Personal Communication James A. Murphy $2 / 12 / 91$.

17. Christian, J.D., Illum, 0.8., Murphy, J. A., Ielente, 1990, 37,651 . 


\section{Appendlx A MICROMax Deseription and Conflguration}

The MICRomax to a Leeds and Northrup procese control oyetem uned for Inline Complexing development. It 1o Imllar to a DCs but does not have certaln DCs foaturee such as poer-to-peer communication. It consiste of a management otat lon and local proceselng otatione (LPUE). The ohort diutances in the ILCM made it feasble to wire all analog ignale to one LPU and all diecrete elgnale to an expanaion unlt wired to the LPU for thlo purpose. Having all the olgnale in one unit made the highly interactive control eystem poselble. All control logle ia performad in the LPU. The oyatem a a whole reade ignale, perfurme calculatione, dioplays and etores data, and procesees alarme and control logle. The MICROMax providee high resolution analog inpute $(15$ blto) and direct reading of thermocouples (wlthin $0.25 \mathrm{c}$ ). Thlo high accuracy aseleted in procese analyolo and development. The ocan rate for 11 elgnalo was tixed at 0.75 noc. Thle provided plenty of epeed for thle proceses. A lieting of portinent analog Inpute tollows thle text. raeh polnt has a deslgnator U I AI XX, otanding for unit 1, analog input $x x$. Inpute 61 through 65 are pulee countere for the turbine matere. Each input has a deelgnated range, whleh provides a Inear converaion to engineering unite. The range lleting is Included in the contiguraton. The control contlguration was entered in a graphical format which the machine complied. An example of graphle programming is ineluded in the contiguration liating.

The overald etructure of the control eyetem le whown in the tollowlng diagram. The flret ANN addition to a calculated feedforward amount baeed on an estimated $2 r$ concentration. It is adjueted by teedback olgnal from an Hr probe. This ame probe leeds corward to a econd ANN addition point which lo alvo leedback adjueted by another HF probe downetream. The logle flow of the two feediorward calculatione de hown atter the control diagram. Water and Nitric acld flowrate etpolnte are caleulated by an overall mase balance. Many of the equat lone were provided by James $A$. Murphy for HF probe ignale and teedeorward additlone. These are described in the atcachment prior to the conflguration.

rauntion

Peeudo polnte are varlables that can be wet by equatione or other numerlcal functione (integration, derivative, fllter, etc). The MiCRomax does not use the -tandard hlerarchy of arlthmet le operatione but evaluatee expreasione in equence - $A+B \cdot C$ - $(A+B) \cdot C$ not $A+(B+C)$ so parenthesis are uaed extenelvely in equat lona to force the desired calculations. In the tollowing lieting, the labele for aach Pp are given at the top of the page, along with a brlef description. The -quation io given with the corresponding number at the bottom of the page. Mout PP' are numerlcal expressions. Some are MICROMAX functione euch as totalleer, rate of ehange, lead/lag, and select. Each loop requires a dedicated PP for lt: execution. Equation abbreviatione are en tollowe:

AI analog input
AO andiog output
CO control output
CN conotant
LS locel otpoint (ot loop number)
PP another poudo point

The 6 Titanium probes that measure HF are central to the aystem. A cuatom made - Lectronice box provides bias voltage and the current through each probe ie converted to a voltage by a current amplifier. A echematic of the box clrcult is Included after the conliguration listing. Each probe signal ls read a a voltage by the MICROMAX and has an asociated thermocouple. Each has an equation that convert the voltage to HF, and another on that corrects this value to a utandard temperature. The probes are numbered $1,2,3,5,6$, and 7 . The position that would be number 4 lo used to read the bia voltage applited to the probes. The probe output ia etrongly dependent on this value eo it ie monltored. 
Conntenten

Conetante are numerleal values that can only be eet by the operator. Often thoee are ueed in equatione Inetead of numerieal values because of the way that the eyetem uees memory. There is no signifleance to the order of conotante and peudo polnte. The teedtorward caloulation for ANN depande on the teed spo. The spo lo monitered but the ignal it noley and contributes to nolee in the control eyetem. An asolgned constant 8 po 10 used Instead and the value lo set at the beglening of the run. The HF value is provlded by the $2 x$ diesolution data. The probe equations uae all the ame conetante exeept for a calibration factor which 1. unlque for ench probe.

control roopen

There are eeven interacting PID loope In the control ayatem. The feed atream and each reagent except final ANN has 1te own tlow controller, and probe ignale control outer eaceade loope. The following diagrame bhow the etsucture and 2 low of logle in the control loope, and the control labele are ohown in the attached conflguration. The MICROMxx allow loope to be tuned with Integral action only if desired. This mode was elected for flow loope after preliminary tevelng. It has the advantages of providing flitering action to the noley 1 low elonale, and oliminates offeet to chieve control at etpolnt. The same tuning mode was Implemented for the probe controlled outer loope with acceptable overall ayatem reuponese times.

several of the poeudo polnte and conetante are solector ewltches which can be aesigned values of zero or one. These are ueed a sectore ln equat lone and control whether part of the equation 10 implemented, for example whether feedback ie combined with a leedforward signal. There are everal forceback equatione. These are used in cascade loope to eat the initial values of the outer loop controlier output to allow bumpiese etertup. This lo accompliehed by nolving for the controller output with other known ignale.

originaliy the equatione converting the outer cascade controller output to a - etpolnt for the ANN flow loop only allowed for ingle-elded control, that le, the amount of ANN could only be increased over the feedforward contribution by teadback. A "normal" controller output would be 0 in this caee. The conflguration was later changed to allow double-nlded control in whlch feedback could add or ubtract from the feedforward ANN. This was accompliohed by ubtracting 50 from the controller output of 0 to 100 , resulting in " "normal" controller output of 50 . This allowed a much clover approach by the foedtorward ayetom. Previoubly, the feedforward contribution had to provide a low estimate of the ANN required to allow the ingle-sided control to work. Now a beet atimate can be used, and the closer the eystem approaches uetpoint with teedforward, the lese teedback correction is required. This is implemented in PP53. PP5 scalas and tranefers the output of the outer loop (4) to the wetpolnt of the middle loop (2) in oimilar double-sided tachion. The target HF io multiplied by co/50 giving "normal" co of 50 and a range of 0 to twlce the tree HF. PPS al oo contain the tactor LS5 in the tadback portion. This is the setpoint for the feedrate. Including this tactor allowe the eysem to respond immediately to otpolnt changea, without having an error propagate through the loop before correction.

\section{Dincrete $1 / 0$}

There are everal discrete I/O pointe in the control conflguration which are primarliy used a logle liaga to difect control of the loops. A box wae wired with awitches to discrete inputs which providod override of automatle shutdown to allow otartup and provide for a forced hutdown. The MICROMAX provided a ladder eyetem for logic programming. The hutdown logle was programmed on a ladder diagram which is included in the configuration liating. The disernte I/O aluo provided control of the outer loop of the caccades for forceback (track) or run etatu to allow bumplese etartup, and control feedforward only or feedforward plue feedback for ANN atartup. This allowed these lmportant functions to be performed without witching display ecreene durling perlods of intense operator demand. A diecrete output was also ueed to control the feed temperature via an on/off loop and a eteam volenold. The eyatem aleo hae control 
relays which are internal status flags. Most of these were used for alarms.

\section{Alarms}

Process alarms and setpoints are given in the configuration listing. These were advisory only and did not perform any automatic functions. Vessel levele and temperatures were alarmed, as were high HF levels in the process. Plugged 1 ines or jammed turbine meters were detected by calculating an expected ratio of control output signal to measured flowrate, and alarming if the ratio fell outside of an expected range.

\section{Displays}

All process data were available on the MICROMAX screen. The gignals were logically arranged into display groups which are given in the configuration listing. These groups could be trended on the screen. The bar/status screen gave an overview of the whole process and direct control over the loop settings and was the primary screen used for operation.

\section{Storage}

All important eignals and peeudo points were recorded on streaming magnetic tape for storage and transfer to Lotus files on a PC for later analysis.

\section{operations}

Each run was performed on a single shift. The actual operation took only 1 to hours, but the setup and calibration of the ingtruments took several hours. The following sheets describe the pre-run checkout, startup, and operation instructions. This system was highly dependent on instruments and the startup of the cascade loops required a complex series of steps. 


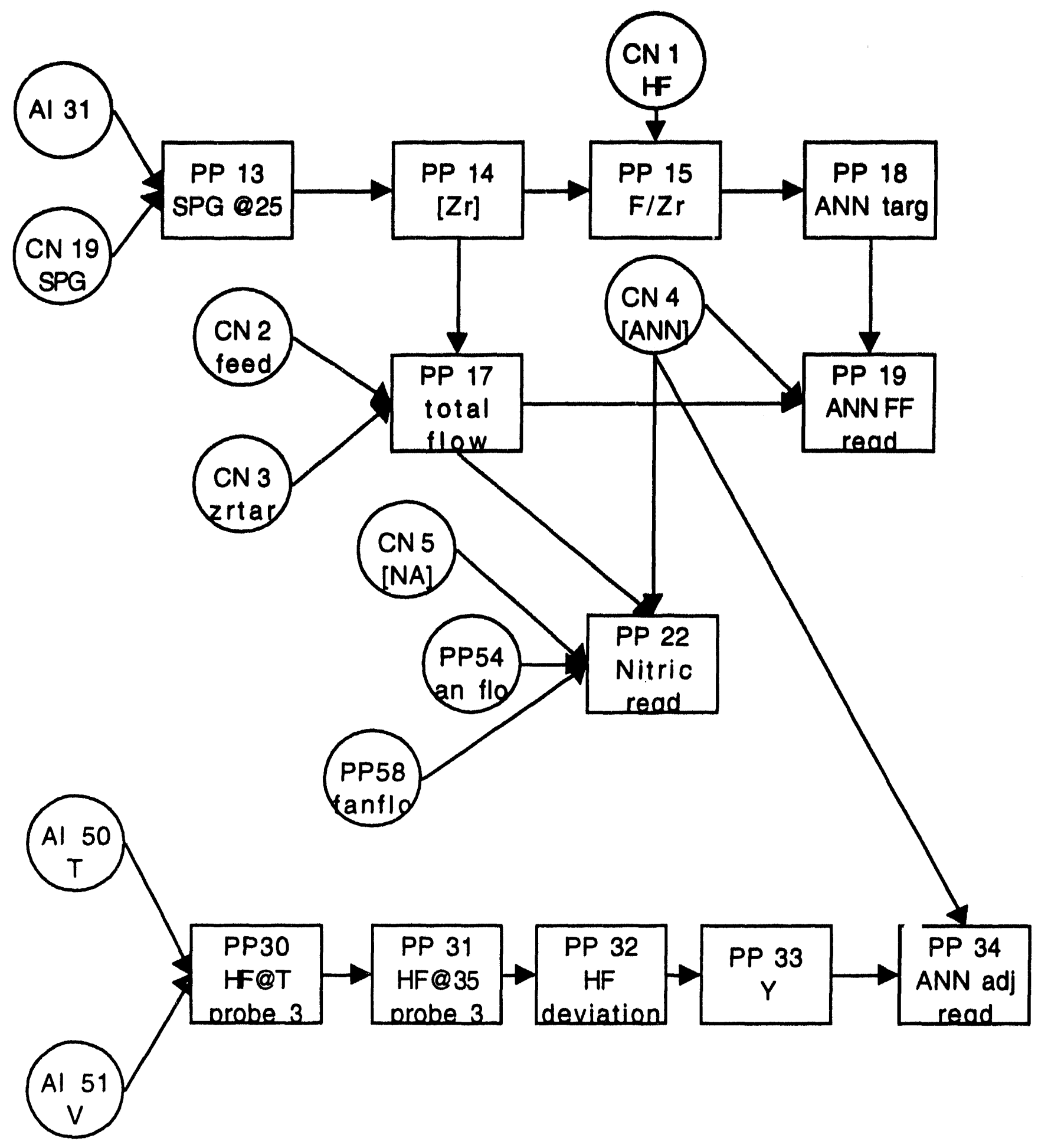




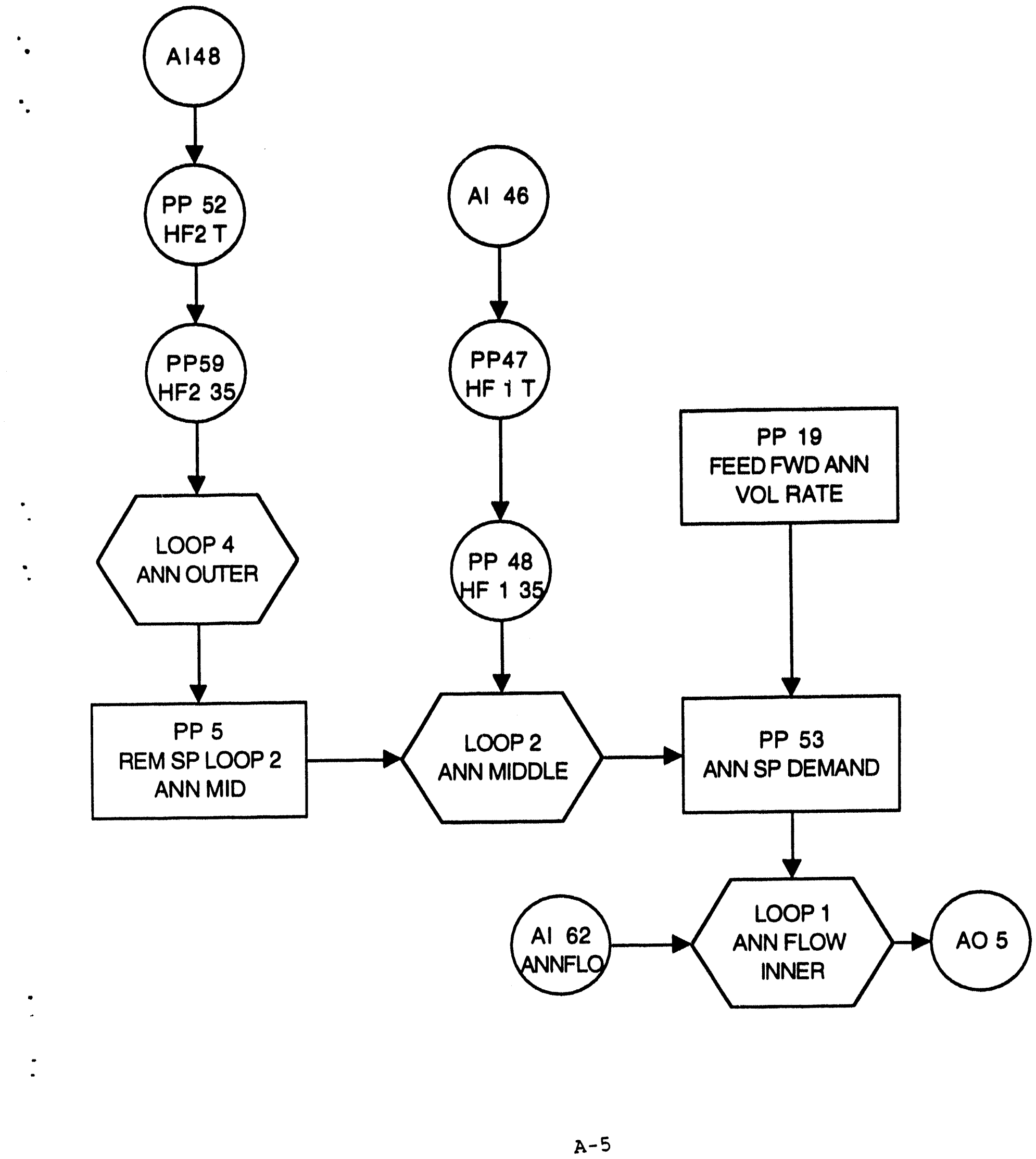



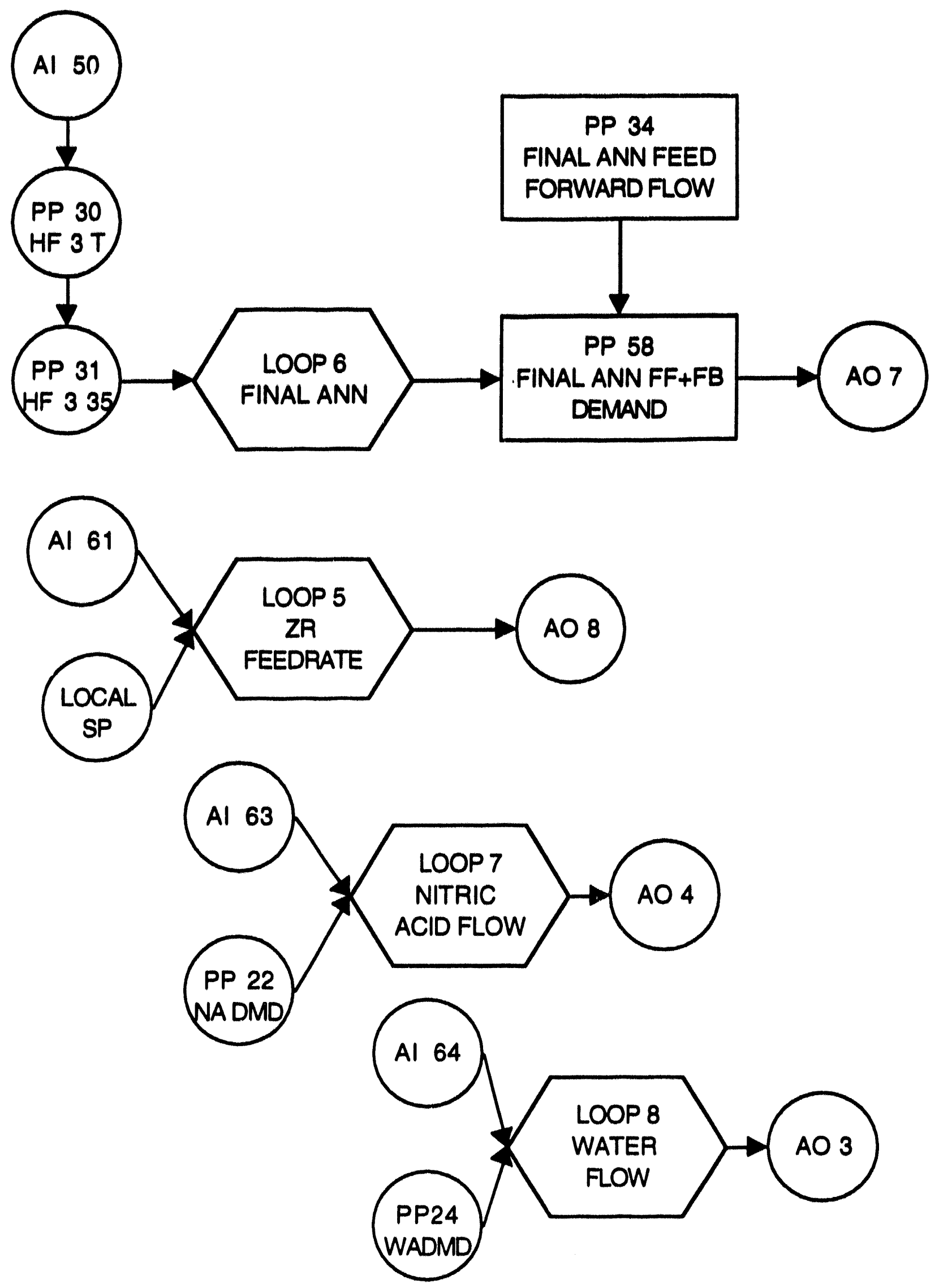

$$
\text { A- } 6
$$


J. A. Murphy

$6-4453 / C P P-637 / 5219$

June 4, 1990

Transmittal of Equations for Control of In-Line Complexing

Attached are the equations and associated supporting information to control the complexing chemistry for the In-Line Complexing Pilot Plant. These equations were generated so to avoid the lengthy iterative calculations which must take place to calculate the free HF in equilibrium with a complex matrix of metals and anions. These equations were created for the GFD type matrix and should not be used to complex other matrixes until I can verify their validity.

\section{J. A. Murphy}

Sr. Scientist, Frocess Development

\section{Attachment Text}

In-Line complexing is identified clearly as the choice method of complexing zirconium dissolver product in the FDP upgrade primarily because of cell apace savings.' This process is downstream of the dissolution equipment and continuously adds the proper additions of aluminum nitrate, nitric acid, and water to bring the free HF of the complexed product to a quantity suitable for stainless stoel vessels downstream of the dissolution facility. Other aspects controlled are the nitrate concentration (for uranium extractability) and final zirconium concentration (for solution stability).

Once the solution has left the dissolution portion of the system, it enters a geometrically favorable vessel where the specific gravity and temperature are measured. These measuremonts are used in conjunction with the initial HF concentration of the feed to calculate the zirconium and fluoride concentration of the solution. These are fed with product flowrate into the computer and flowrates of the three complexing solutions, aluminum nitrate, nitric acid, and water, are generated. The solution is initially slightly undercomplexed to a degree adequate for the anticipated error of the conversion of specific gravity to fluoride and zirconium concentrations to ensure overcomplexing does not occur. The undercomplexed solution is mixed, allowed some time to reach equilibrium and measured by a titanium electrode for free HF. The free HF concentration is fed into the computer and a flowrate of aluminum nitrate is calculated to complete the complexing to the goal free HF of 0.045 Molar at 35 degrees $C$. This goal will be optimized following some corrosion and stability laboratory tests.

The solution will run past another titanium electrode where a second adjustment will attempl to finalize the complexing within an operating envelope. However, it will be attempted to finish all adjustments with only one adjustment. The final solution will be measured in a holding tank and adjustment lines into these vessels will allow correction of the free HF if necessary to prepare the product for pilot plant calcine feed.

In the past, in order to complex a zirconium dissolver product, a computer program used an iterative calculation method to converge all the equilibria and, a solution with a number of ions, takes several thousands of calculations. This is an adequate way to determine the complexing needs for a bulk tank, but inadequate for a continuous process which changes from time to time. The equations generated in the following sections will allow the Micromax computer, which has simple mathematical capabilities, to calculate the needed additions in a real-time fashion.

The equations will be broken up into two sections. First, the initial complexing which will 
intentionally undercomplex the dissolver product. Second, the adjustment equations are given to finalize the free HF to the proper concentration.

Many of these equations are derived using laboratory data because the free HF equilibrium equations appear to calculate about 10.15 percent low as compared to the titanium electrode. Studies of the titanium electrode are continuing, to give assurance that the reading is proportional to only free HF.

\section{Initinl Complexins}

Initially, the computer will need three inputs to properly generate the initial flowrates. These inputs are the specific gravity, temperature, and the initial HF concentration. It is important that these measurements be as accurate as possible to reduce the associated error in the flowrate calculations. The first equation converts the specific gravity and associated temperature to a normalized specific gravity at 29 degrees C. This equation was generated by $L$. L. Burger, et al, and is rewritten to solve for upecific gravity at 25 degrees $C$ as follows:

Equation I Sp-Gr $2 s=\left(D_{1}+0.0036 \cdot .000145 * t\right) /(1.0125 \cdot 0.0005 * \imath)$

where $D_{1}$ is the density at the moasured temperature, $t$ in degrees $C$.

The next step is to calculate the zirconium concentration as described in equation 2.

Equation $2[\mathrm{Zr}]=\left(\mathrm{Sp}-\mathrm{Gr}_{25} \cdot 0.0066 *[\mathrm{~F}] \cdot 0.99707\right) / 0.1367$

where $[F]$ is the initial HF concentration in the dissolvent feed and $[\mathrm{Zr}]$ is the molarity of zirconium in solution (ref 3,4 ). These equations were generated prior to the development of highly analytical specific gravity instrumentation as available in the Remote Analytical Laboratory and were specific for a different flowsheet than GFD. This is one area where improvement is possible if these density equations would be reevaluated using current technology and current flowsheet compositions.

These calculations are necessary to properly calculate the fluoride (same as initial HF) to zirconium molar ratio. This ratio affects the quantity of aluminum that is needed to complex the free HF. This simple equation is:

Equation $3 \mathrm{~F} / \mathrm{Zr}=[\mathrm{F}] /[\mathrm{Zr}]$

The zirconium target concentration must be stipulated to continue. The product coming out of FDP is currently 0.49 molar zirconium'. This concentration is necessary to ensure stability in the tank farm. (There is some hope this concentration will be increased because of anticipated increased stability after running through FPR's first cycle and an increase in the minimum temperature in the tank farm to 25 degrees $C$. These two changes may take the zirconium concentration in the product to 0.68 molar in the final GFD product ${ }^{6}$.) Because there is an anticipated $7 \%$ volume addition expected for adjustments, the initial target will be set at 0.524 molar zirconium. This target calculates the final volume the product must be diluted to as follows:

Equation 4 final flowrate $=([\mathrm{Zr}] / 0.524) * \mathrm{ZrDP}_{\text {nownie }}$

For a fixed final zirconium concentration, the targeted aluminum concentration is proportional to the $[\mathrm{F}]$ to $[\mathrm{Zr}]$ molar ratio from equation 3 . The slope and intercept of this linear equation are cubic functions of the targeted free HF concentration and should be calculated prior to insertion into the 
Micromex calculational control loop. This equation will allow the free HF to be changed without redoing all the equations. Equation 5 shows the function to calculate $\mathrm{m}$, the slope. Equation 6 shows the function to calculate $b$, the intercept. Equation 7 shows the function to calculate the final aluminum concentration. The targeted free HF based on equilibrium equations used will probably be 0.045 molar but, because of the differences between the equilibrium equations and the titanium olectrode, the actual electrode terget is 0.053 Moler free HF.

$$
\begin{aligned}
& \text { Equation } 5 m_{\text {dlopp }}=1.4374 \cdot[H F]^{2} \cdot 0.4997 \cdot[H F]+0.2672 \\
& \text { Equation } 6 b_{\text {inimuapl }}=-899.6 \cdot[\mathrm{HF}]^{3}+168.8 \cdot[\mathrm{HF}]^{2} \cdot 11.7992 \cdot[\mathrm{HF}] \cdot 0.8281 \\
& \text { Equation } 7|A|]=m \cdot[F] /[Z r]+b
\end{aligned}
$$

where [HF] is the targeted free HF at 35 degrees $C,|A|]$ is the final aluminum concentration needed, $[F]$ is the initial feed HF concentration, and $[\mathrm{Zr}]$ is the zirconium concentration calculated from the density measurements. The amount of aluminum needed to complex the excess froe HF is, a defined above, is a function of the desired final froe HF concentration and a function of the fluoride to zirconium ratio. Several fits were attempted, but the best statistically significant fit was the above relationship. The equations for the slope and intercept were calculated using several subsets of date with different final free $H F$ 's and $F / Z_{r}$ ratios. These set up a family of curves which individually were linearly fitted with respect to the $F / Z r$ molar ratio and the final needed aluminum concentration. Each subset's coefficients were fitted to many functions of final HF concentration until the cubic relationship was found to be the best for both the slope and intercept in relationship to the targeted free HF molarity. It is important to note that the first two cubic equations 5 \& 6 should be calculated prior to placine equation 7 into the Micromax computer. Equations 5 \& 6 are only needed when changing the initial free HF target.

These equations were generated using data calculated by a program which used the HF equilibrium constants and varied the $\left[\left.H F\right|_{\text {intud }}\right.$ concentration and the density measurements. Because the data calculated were generated by a computer program, a good $R^{2}$ factor was achieved as follows. $R^{2}$ on the equation for the slope was 0.9998 , the $R^{2}$ on the equation for the intercept was 0.9999 . The $R^{2}$ on the linear equations for each [HF] concentration was 1.0 . The combined fit was not calculated simply because the slope and intercept fits were so good and the overall fit of the three cquations as a whole is better than 0.999 .

Once the $|A| \mid$ (aluminum molarity) is calculated, the amount of aluminum to add is calculated by multiplying the $|A| \mid$ by the final flowrate and dividing by the concentration of the aluminum feed concentration, $[A N N]$, as follows in equation 8 :

Equation 8 Aluminum nowrate $=|A|] *$ final nowrate $/[A N N]$

The nitric acid concentration is next to calculate and uses a target of approximately 2.0 molar. Realize the final targeted nitrate concentration is set for 2.4 molar to allow for good uranium extractability. However, because there are still additional adjustments with aluminum nitrate, the nitrate is adjusted initially low. The nitric acid concentration is naturally related to the nitrate concentration desired with the amount already added by the aluminum in equation 8 subtracted out as follows:

Equation $9 \mathrm{HNO}_{3 \text { nowrate }}=(2.0-3 *[\mathrm{Al}]) *$ final flowrate $/[\mathrm{HNO}$,$] .$ 
This and other equations aseume that an aluminum baket will not be used in the pilot plant. Some adjustments to these equations may be needed to allow for the aluminum from the baket material used in the actual plant.

The last step of the initial complexing equations is the water flowrate. This is a simple subtraction of the total added volume from the needed volume for zirconium stability. Equation 10 explaina.

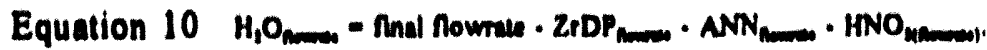

Although these equations are simple, they nevertheless removed many of the iterative calculations necessary to initially complex a solution. Some of the targets a stated in earlier parts, may need to be adjuated when setual plant data are available 10 optimize the solution's chemiatry.

\section{Adiuntment Routines}

These routines are based on having a calibrated titanium electrode reading (shown how to do later) and a calibrated temperature reading. These equations were not trivial $10 \mathrm{get}$ and are somewhat limited in scope. They should however, work adequately well with the in-Line complexing of GFD product. The equations will need revision if they are to be used on poisoned product or solutions out of the operating ranges set for the GFD.

The first equation deals with the proper calibration of the Titanium electrode. Currently, the majority of the electrodes in the In-Line complexing pilot plant module contain the Ti-0.2\%Pd electrode material. The equation given below is good for these types only. A similar equation will be generated by D. B. Illum soon for the commercially pure titanium electrode. The calibration of the electrode involves accurately measuring the electrode voltage in $\mathrm{HF} \cdot \mathrm{HNO}$, calibration solution. The temperature must also be measured. A calibration factor, $f$, is calculated as follows. For this equation, an example will be used where an HF solution [HF] is measured at temperature 1 and with a voltage volts.

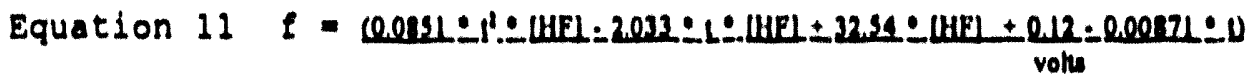

This factor, $f$, can be used to directly calculate the free HF in solution at any temperature by using equation 12.

Equation $12 \quad[\mathrm{HF}]=((\mathrm{v} * \mathrm{fl})+0.00871 \cdot 1 \cdot 0.12) /\left(0.0851 \cdot t^{2} \cdot 2.033 * 1+32.54\right)$

where $t$ is measured degrees $C, v$ is measured volts, and $[H F]$ is free HF in solution at the measured temperature, $t$. Both of these equations are the same except solved for different factors. When analyzing this equation, a relationship was found by observing the subsets of tomperature data which created a family of curves. Each individual curve at a given temperature exhibited a linear relationship between current and free HF in solution. The coer icients were then examined and the slope was found to be a cubic function of the temperature and the intercept was a linear function of temperature. Once the entire equation was evaluated, the residual standard deviation publiahed' for this equation was 0.00088 molar free $H F\left(R^{2}=0.9992\right)$.

The next calculation is to normalize the free HF measurement to a standard temperature to allow correct adjustments. 10 solutions were accurately prepared and moasured by the titanium olectrode at 
varying temperatures (from 251049 degrees C) to get the next equation which normalizas the mesured free HF at process temperature to the froe HF at 39 degrees C. The expected temperature at the fint edjustment point is 40 degress $C$. The linear equation's slope is a function of the messured temperature.

Equation $13[\mathrm{HF}]_{33}=[-0.01434 * 1+1.4934] *[\mathrm{HF}]_{1}+0.00044$

Each subset of date had a linear fit with free HF at the siven temperature and the free His at 35 degress C. The coomcients from the fumily of eurves were fitted to temperature. The slope was found to be linear with respect to temperature. However, the intereept remained constent (virtually zero). This equation hes a residual standard deviation on the estimated [HF] at 35 degrees $C$ of 0.00053 molar.

The next equation is a bit more tricky to use and will be done in a couple of stops to meke it easier to underntand. About 15 slightly undercomplexed solutione were titrated with 2.22 Moler $A \mid(N O,)_{1}$, to a measured free HF of approximutely .035 molar at 35 degrees C. They were actually titrated 10 a normalized voliage which happened to be about 039 moler HF normalized to 39 degrees $C$. The relationship found for the edjustment part of the In-Line complexing is a proportionality of the free HF which needs to be complexed with the molar ohange in aluminum neceseary to complex it. The equation defined has several parts to it. Fint, the " $x$ " parameter must be defined. This factor is the mosured free HF normalized to 35 degrees $\mathrm{C}$ minus the tarseted 0.045 Moler free HF at 35 degrees C.

Equation $14 x=[\mathrm{HF}]_{, 1}=0.049<\ldots . . .(\mathrm{HF}$ Iarget)

Next is 10 calculate the function for the volume of aluminum to add, but to simplify the calculation, $y$ is calculated firat.

Equation $15 y=612.91 \cdot x^{3} \cdot 79.437 * x^{2}+6.062 \cdot x \cdot 0.00037$

Equation 16 ANN nowrate - y " product nowrate / ([ANN] - y)

where the product nowrate is the nowrate at the adjustment point (prior to adjustment AI(NO,), addition) and $[\mathrm{ANN} \mid$ is the stock aluminum nitrate reagent concentration.

These relationships above wure found by analyzing the effects of a different starting free HF and different volumes on aluminum needed to complete complexing based on atarting relationahip of

$$
\frac{n A L}{V_{\text {nnd }}}=f(x)=y
$$

where $n A l$ is the number of moles of aluminum needed and Vfinal is the volume being adjusted. The relationship each had on the complexing calculation was not known. Some information was known about the relatively weak-complexing ability of the aluminum ion in comparison to the strong-complexing zirconium ion in the syatem. Because zirconium was such a stronger complexant, the free HF above the goal free HF (the difference as stated in Equation 14) was primarily the HF titrated with the aluminum. In other words, the aluminum did not atrip of much of the fluoride from the zirconium but enough the linear function of aluminum needed and free HF measured was not adequate. However, the cubic function was. It was also known that the aluminum needed was a function of the volume to be complexed. This function was calculated after the insertion of the cubic 
function of free HF to complex was added. The lat pert of the equation was the conversion from aluminum volume to moles by using the duminum nitrate concentration.

This equation is sood for an initial tree HF of up 100.11 moler and for adjuating the solution to 0.045 moler free HF at 35 degrees $C$. The same equation can be used for calculating both adjustment points since they are similar. The standerd error on this equation is the highest of all the equations and umounts 10 about $9 \%$, which results in a complexing errof of 0.0022 moler free HF. The size of this efror was not unexpected since these equations were generated using sctual laboratory results where some measurement errors were probable. Using different concentrations of aluminum nitrate from 2.0 102.3 does not severely afreet this equation's effectiveness on proper complexins.

Most of these equations were generated in the laboratory where conditions were controlled (such as stirring rate, stabilization time, etc.). There is sossibility that some bises in the free HF complexine will occur in the continuous system. This will be handled by adjusting these equations with sotud pilot plant dats to schieve proper complexing and/or modifying the equipment configuration as needed.

This letter completes, for now, the ection needed to statistically senerate equations eapable of properly complexing arreonium dissolver product in the OFD In-Line complexing pilot plant without iterative convergence routines. In the future, en improved equation correlating airconium concentration with the measured specific grevity of the zirconium dissolver product will be determined for specific OFD compositions. This is expected to improve the eccuracy of the initial complexing.

References:

I. R J Kirkham, RJK.5.89 10 M. D Staiger, "Preliminary In-Coll Equipment Flowsheets for FDP Upgrade Options", dated October 31. 1989, still to be issued

2. L L Burger, I M Rehn, H R Schmidl and C M Slansky. Bropenies o C the Syatem: Urnay

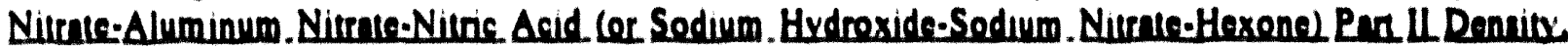
Apparens Moles Volume and Viscours. HW.11276, March 1949.

3 J. F Krupa, JFK-2.84 to J D Christian. "Density Equation for Fluorinal B/Cd Flowsheet Product", daled March 6, 1984

\section{Hydroflueric Acid reference manual Copyrighi 1978, Allied Chemical Corporation.}

5. J. A Murphy, Murp.1.89 to J A Rindneisch, "Flowsheel Changes for FDP Campaign 44", dated March 8, 1989, (confidential allachment)

6 J. A Murphy, Murp-2.89 10 C B. Millet. "Preliminary Raminate Solution Compositions for Present FDP and Upgrade GFD Dissolution Processes," dated April 30, 1990.

7. J. D. Christian, D. B. Illum, and J. A. Murphy, Molll Electroder Lor Continuour Amperometric Moururement of Eree Hydrofluoric Aaid In. Complexed. Acidic. Solutions, Talenta, 31.651.654 (June 1990). (Note that data and equations in this paper for the Ti.Pd electrodo are for the Ti-0.2\% Pd nominal composition, the composition stated. Ti.2\% Pd, is incorrect.) 


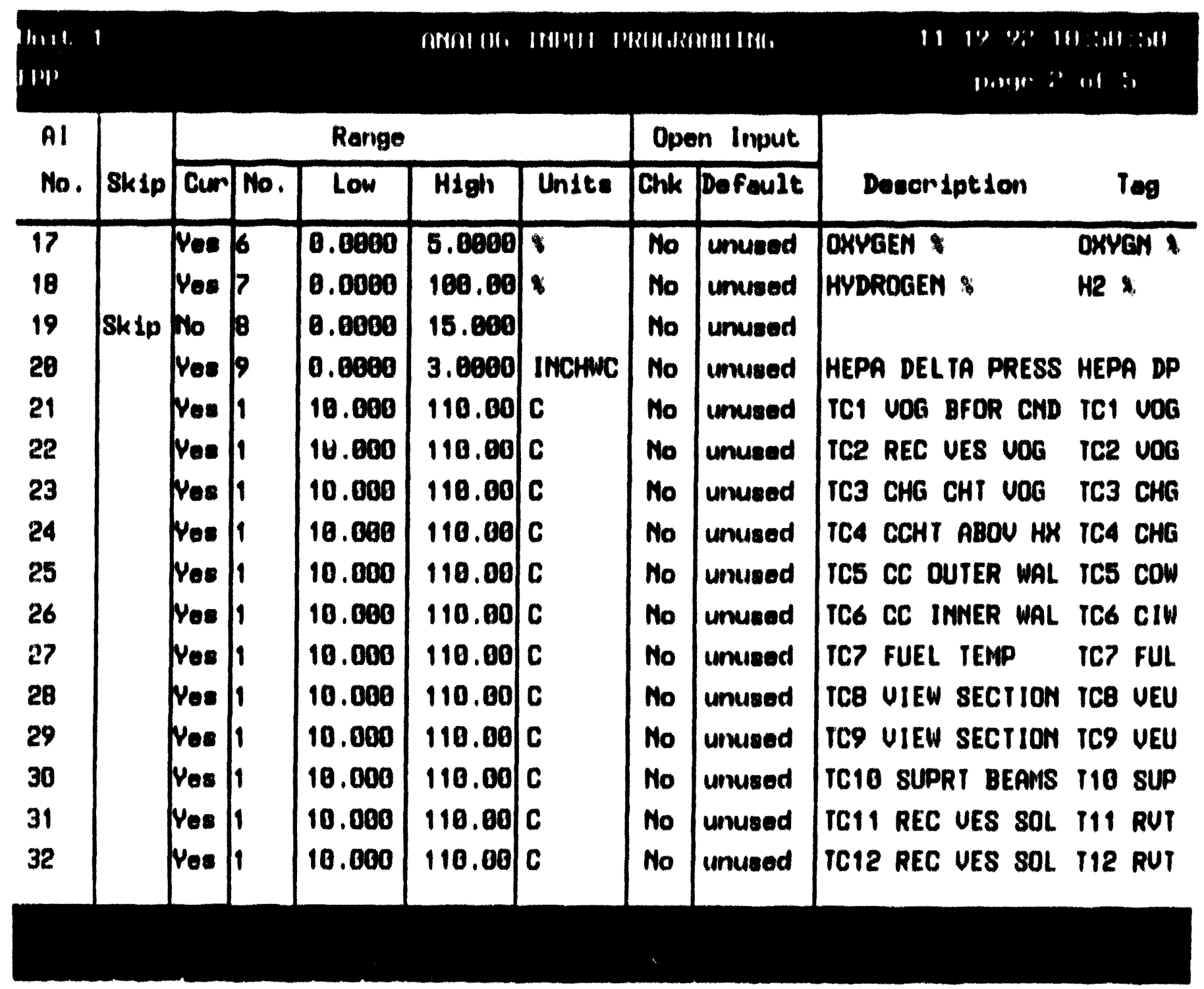




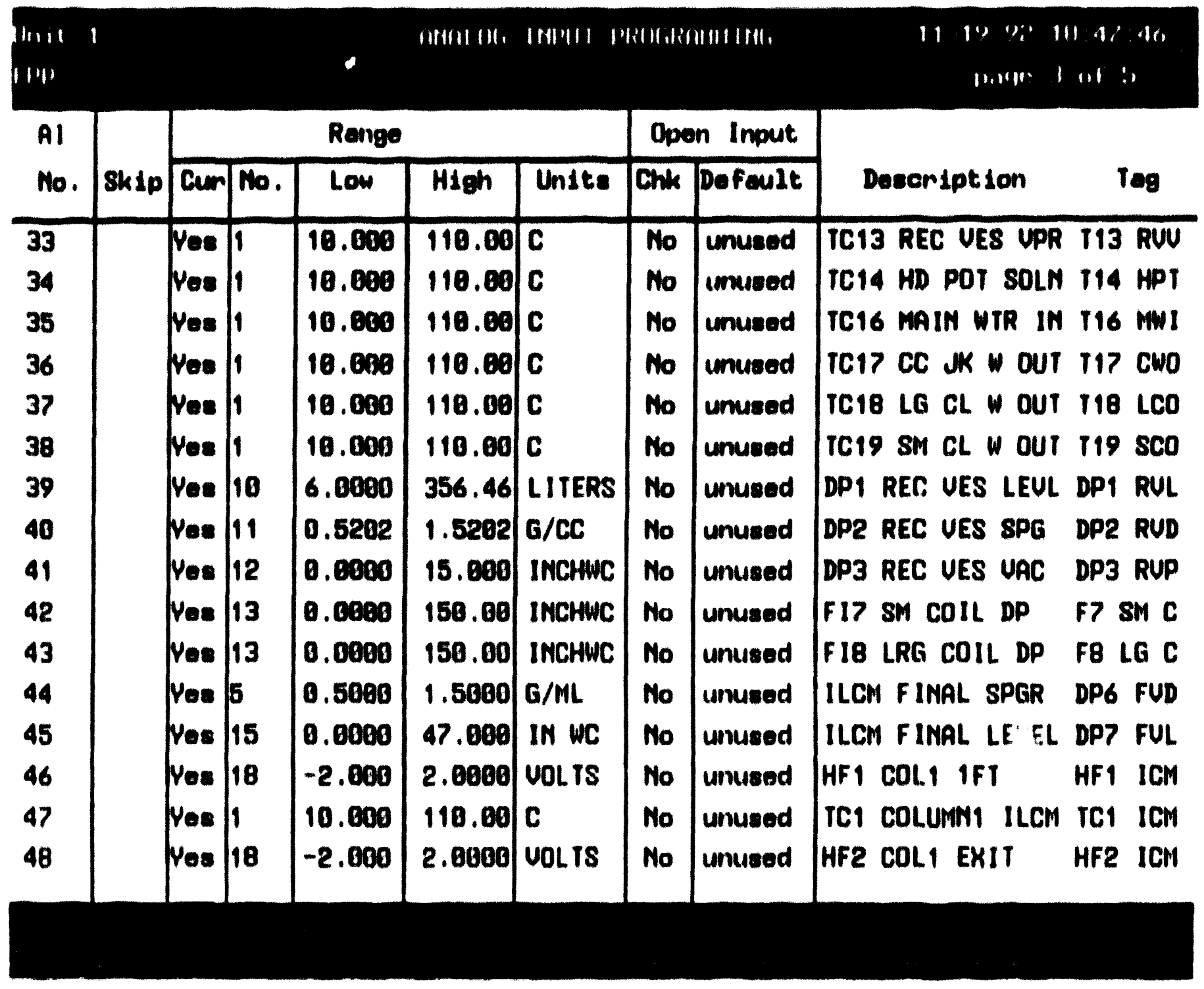




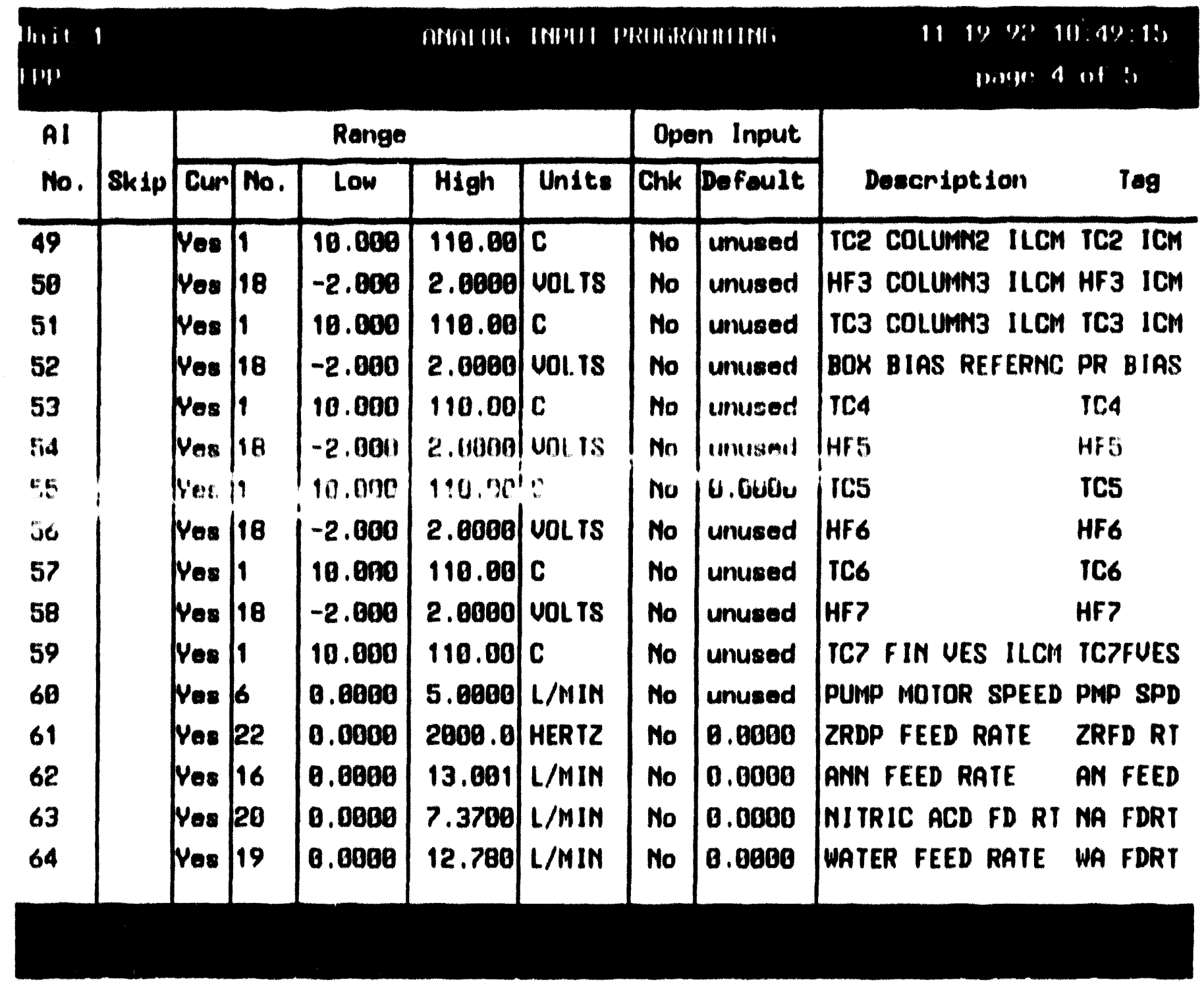




\begin{tabular}{|c|c|c|c|c|c|c|c|c|}
\hline Rng. & I t...te Typo & $\begin{array}{l}\text { Lovl laved } \\
\text { Low }\end{array}$ & $\begin{array}{l}\text { d Range } \\
\text { High }\end{array}$ & $F / C$ & $\begin{array}{l}\text { Dir/ } \\
\text { Ind }\end{array}$ & $\begin{array}{l}\text { Electrice } \\
\text { Low }\end{array}$ & $\begin{array}{l}1 \text { Range } \\
\text { High }\end{array}$ & $\begin{array}{l}\text { Elect } \\
\text { Units }\end{array}$ \\
\hline 1 & $\mid$ Trpe K TC & 10.000 & 110.00 & $\operatorname{deg} \mathrm{C}$ & Dir & 0.0080 & 0.0800 & v \\
\hline 2 & Volts & 0.0800 & 180.00 & $\operatorname{dog} C$ & Ind & 1.0000 & 5.0090 & $\mathbf{v}$ \\
\hline 3 & Volts & 3.0000 & 78.768 & deg C & Ind & 1.8080 & 5.8880 & $u$ \\
\hline 4 & Uolts & 8.2800 & 320.00 & deg C & Ind & 1.8080 & 5.8880 & u \\
\hline $\mathbf{5}$ & Volts & 0.5890 & 1.5000 & deg C & Ind & 1.8080 & 5.8000 & $v$ \\
\hline 6 & Volts & 0.0000 & 5.8880 & deg C & Dir & 0.0000 & 5.0000 & $\mathbf{U}$ \\
\hline 7 & Range $X$ & 0.0000 & 100.00 & deg F & Ind & 0.0000 & 5.0000 & u \\
\hline 8 & MIllivolt & 0.8080 & 15.800 & deg C & Ind & -3.659 & 25.150 & $m U$ \\
\hline 9 & Volts & 0.0880 & 3.0980 & deg C & Ind & 1.0808 & 5.0800 & v \\
\hline 10 & Volts & 6.0000 & 356.46 & deg C & Ind & 1.0080 & 5.0000 & $\mathbf{u}$ \\
\hline 11 & Volts & 0.5202 & 1.5202 & deg C & Ind & 1.0000 & 5.8000 & v \\
\hline 12 & Volts & 0.0800 & 15.080 & deg C & Ind & 1.0000 & 5.8800 & u \\
\hline 13 & Volts & 0.0000 & 150.00 & deg C & $d$ & 1.0000 & 5.0400 & $v$ \\
\hline 14 & Volts & 0.8000 & 60.000 & $\operatorname{deg} C$ & and & 0.0888 & 5.8001 & $u$ \\
\hline 15 & Volts & 0.0080 & 47.080 & $\operatorname{deg} C$ & Ind & 1.0808 & 5.0000 & $\mathbf{u}$ \\
\hline 16 & Pulse Pos & 0.0800 & 13.001 & $\operatorname{deg} C$ & Ind & 0.0080 & 1600.0 & $\boldsymbol{u}$ \\
\hline
\end{tabular}




\begin{tabular}{|c|c|c|c|c|c|c|c|c|}
\hline \multirow[b]{2}{*}{ Rng. } & \multirow[b]{2}{*}{ Input Type } & \multicolumn{4}{|c|}{ AMALOG RANGE PROGRAMMING } & \multicolumn{3}{|c|}{$\begin{array}{l}11-19-92 \text { 18:43:47 } \\
\text { PAGE } 2 \text { OF } 4\end{array}$} \\
\hline & & $\begin{array}{l}\text { Displayer } \\
\text { Low }\end{array}$ & $\begin{array}{l}\text { d Range } \\
\text { High }\end{array}$ & $F / C$ & $\begin{array}{l}\text { Dir/ } \\
\text { Ind }\end{array}$ & $\begin{array}{l}\text { Electrical } \\
\text { Low }\end{array}$ & $\begin{array}{l}1 \text { Range } \\
\text { High }\end{array}$ & $\begin{array}{l}\text { Elect } \\
\text { Units }\end{array}$ \\
\hline 17 & Pulse Pos & 0.0000 & 14.014 & deg C & Ind & 0.0900 & 1680.0 & II \\
\hline 18 & Uolts & -2.000 & 2.0000 & $\operatorname{deg} C$ & Dir & -2.000 & 2.0009 & u \\
\hline 19 & Pulse Pos & 0.0900 & 12.780 & deg C & Ind & 0.0000 & 1600.0 & $u$ \\
\hline 20 & Pulse Pos & Q. 0000 & 7.3700 & deg C & Ind & 0.0090 & 3600.0 & $u$ \\
\hline 21 & Pulse Pos & 0.0000 & 14.150 & deg C & Ind & 0.0000 & 1600.0 & u \\
\hline 22 & Pulse Pos & 0.0000 & 2000.0 & $\operatorname{deg} C$ & Ind & 0.0900 & 2000.0 & U \\
\hline 23 & MO INPUT & 0.0000 & 0.0000 & deg C & Dir & 0.0000 & 0.0000 & u \\
\hline 24 & INO INPUT & 0.0000 & 0.0000 & $\operatorname{deg} C$ & Dir & 0.0000 & 0.0000 & U \\
\hline 25 & MO IMPUT & 0.0000 & 0.0000 & $\operatorname{deg} C$ & Dir & 0.0000 & 0.0000 & U \\
\hline 26 & NO INPUT & 0.0000 & 0.0000 & $\operatorname{deg} \mathrm{C}$ & Dir & 0.0900 & 0.0000 & $u$ \\
\hline 27 & NO IMPUT & 0.0000 & 0.0000 & $\operatorname{deg} C$ & Dir & 0.0000 & 0.0000 & u \\
\hline 28 & NO INPUT & 0.0000 & 0.0080 & deg C & Dir & 0.0000 & 0.0000 & u \\
\hline 29 & MO IMPUT & 0.0080 & 0.8000 & deg C & Dir & 0.0800 & 0.8080 & $u$ \\
\hline 30 & NO INPUT & 0.0000 & B. 0000 & deg C & Dir & 0.0000 & 0.0000 & U \\
\hline 31 & MO INPUT & B. 0000 & 0.0000 & deg C & Dir & 0.0000 & B. 0000 & u \\
\hline 32 & NO INPUT & 0.0000 & 0.0900 & deg C & Dir & 0.0090 & 9.0000 & u \\
\hline
\end{tabular}




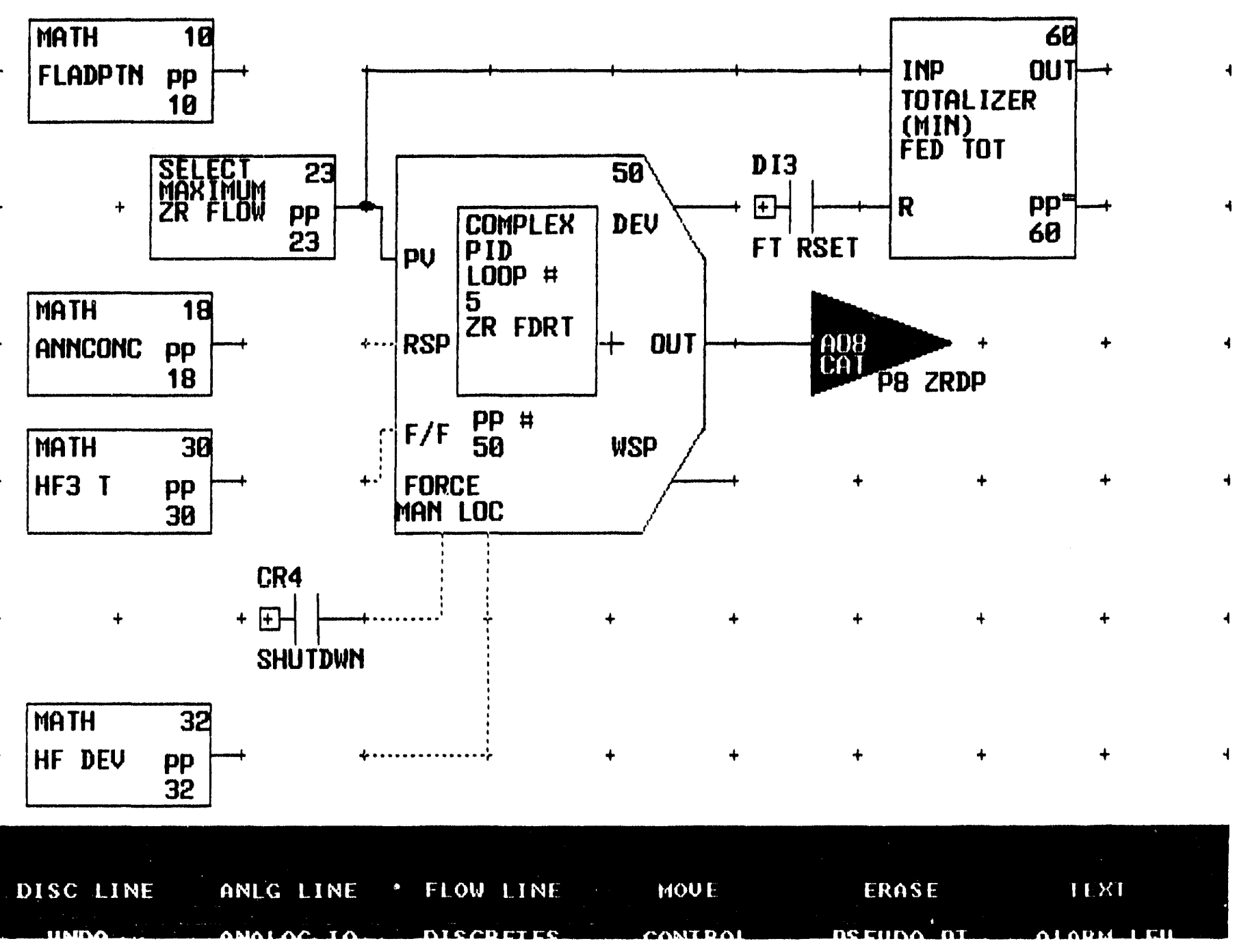




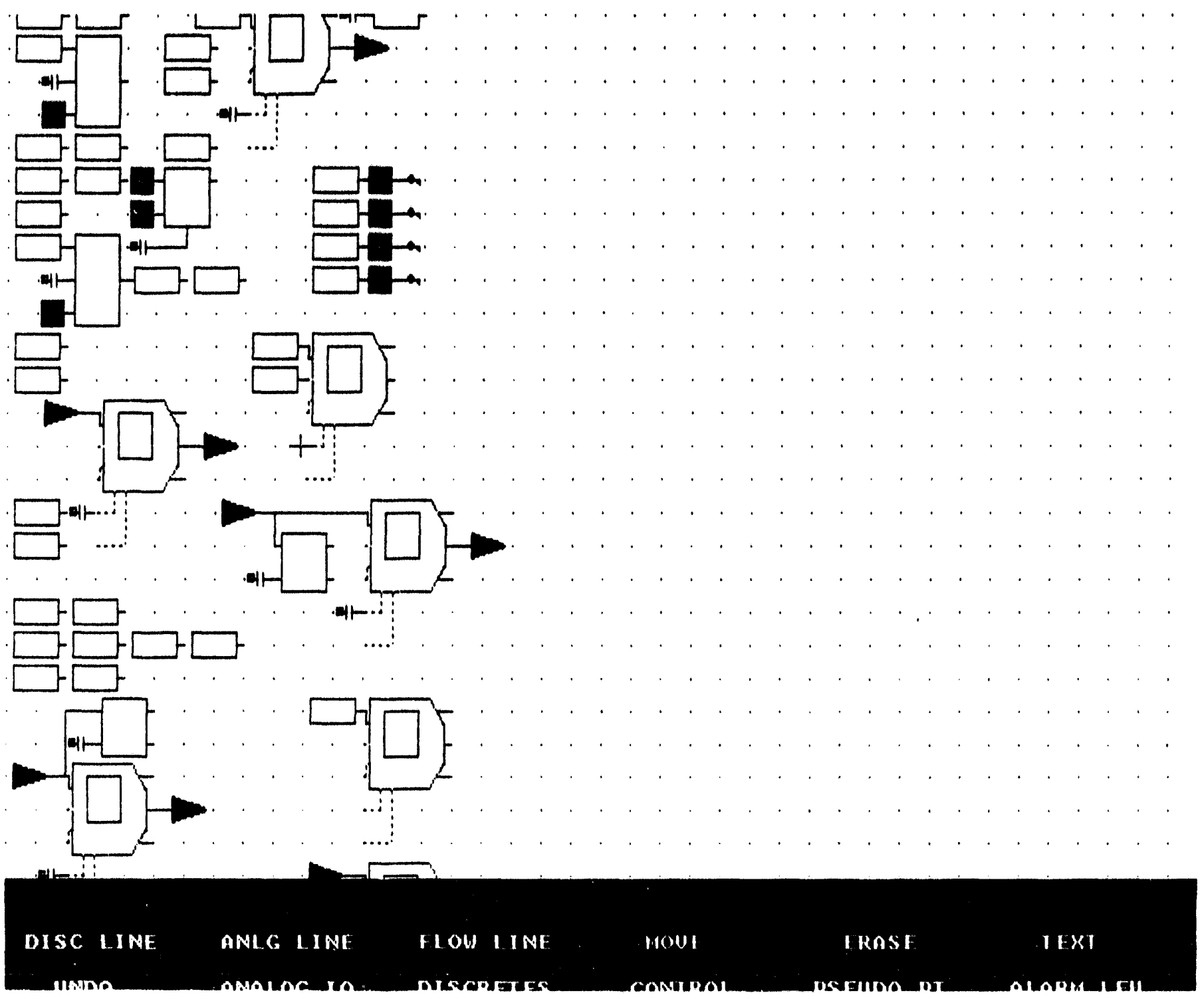


Attachment II

DAC-11-92

November 5, 1992

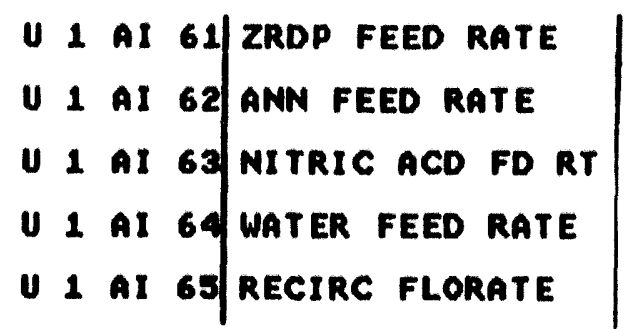

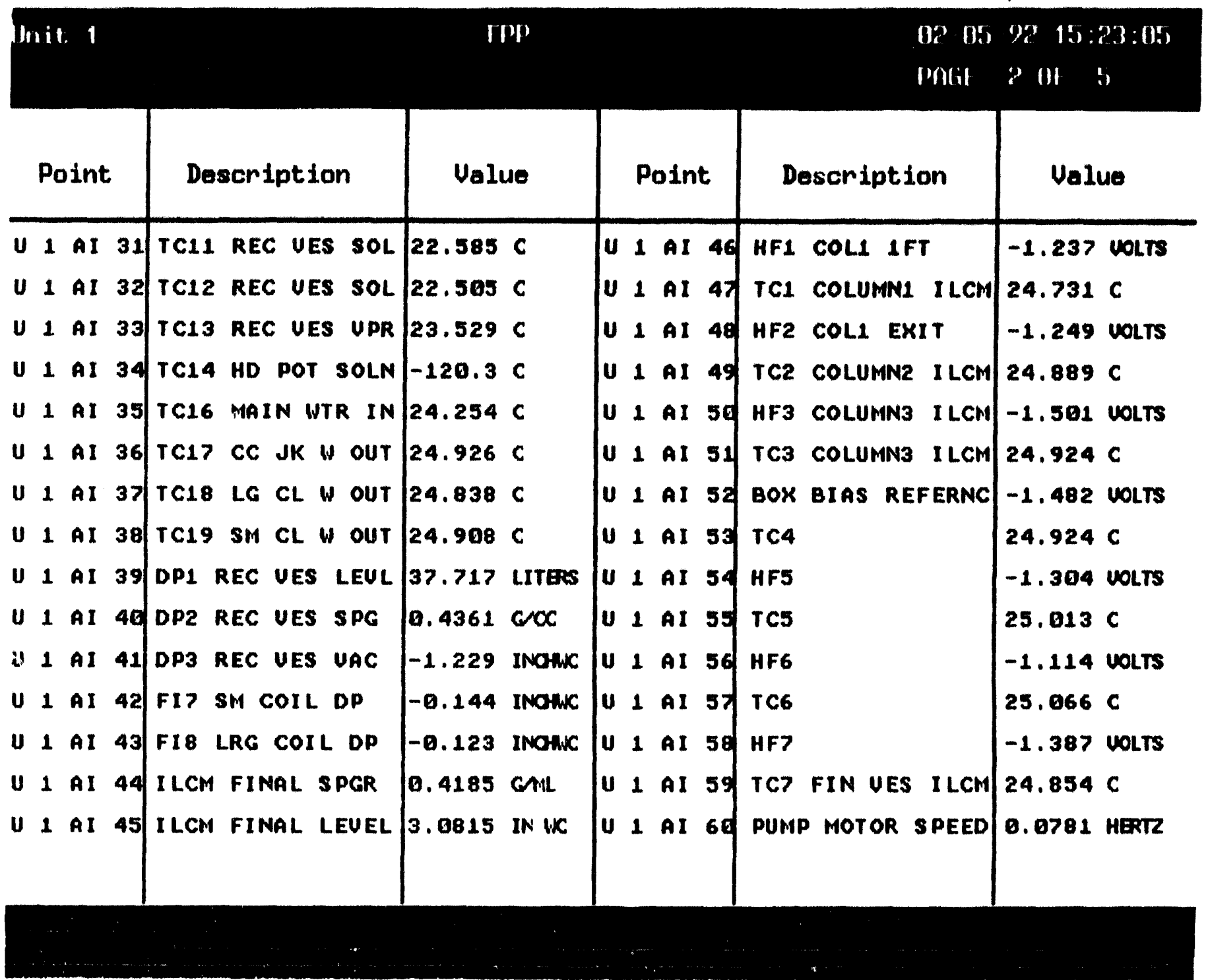




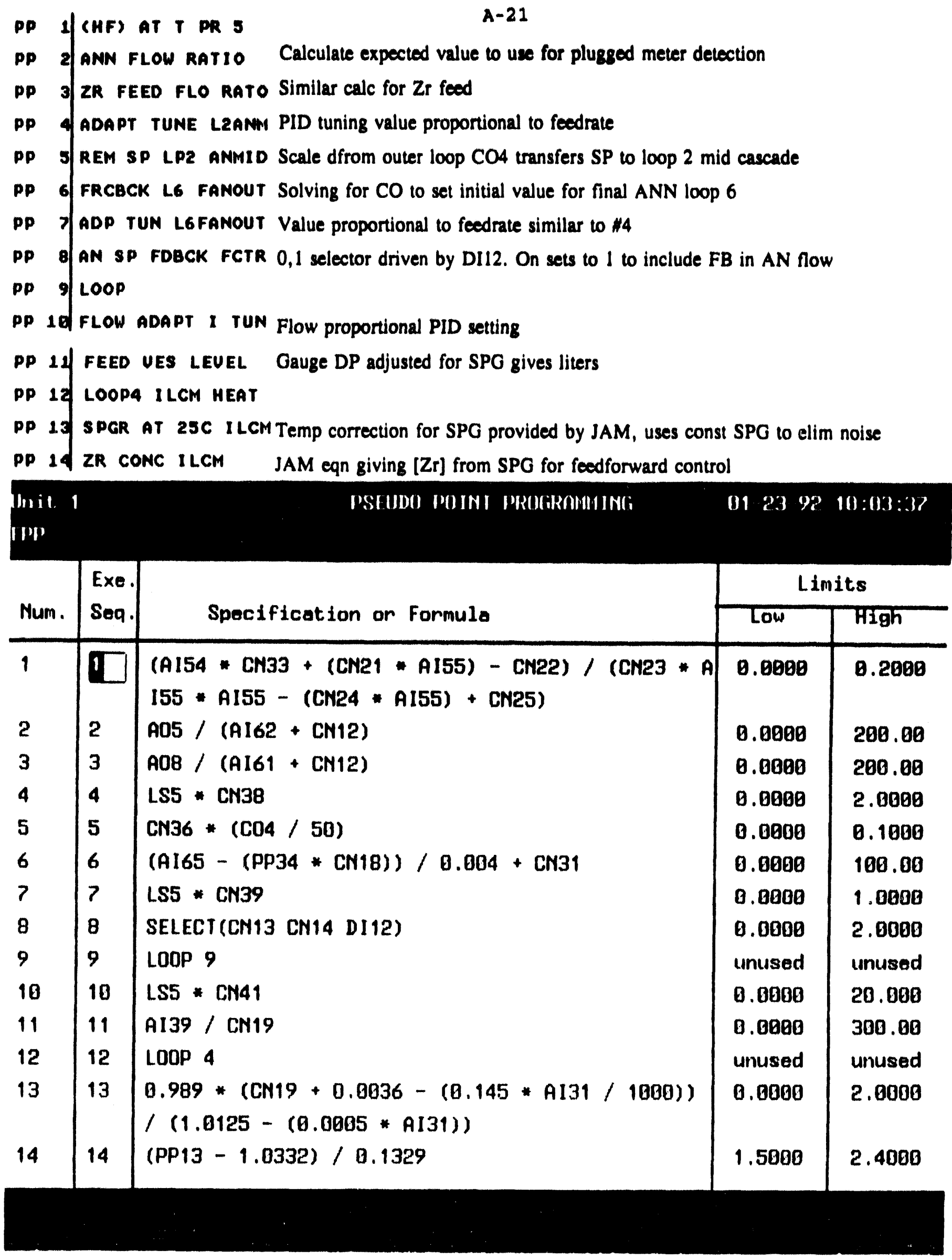




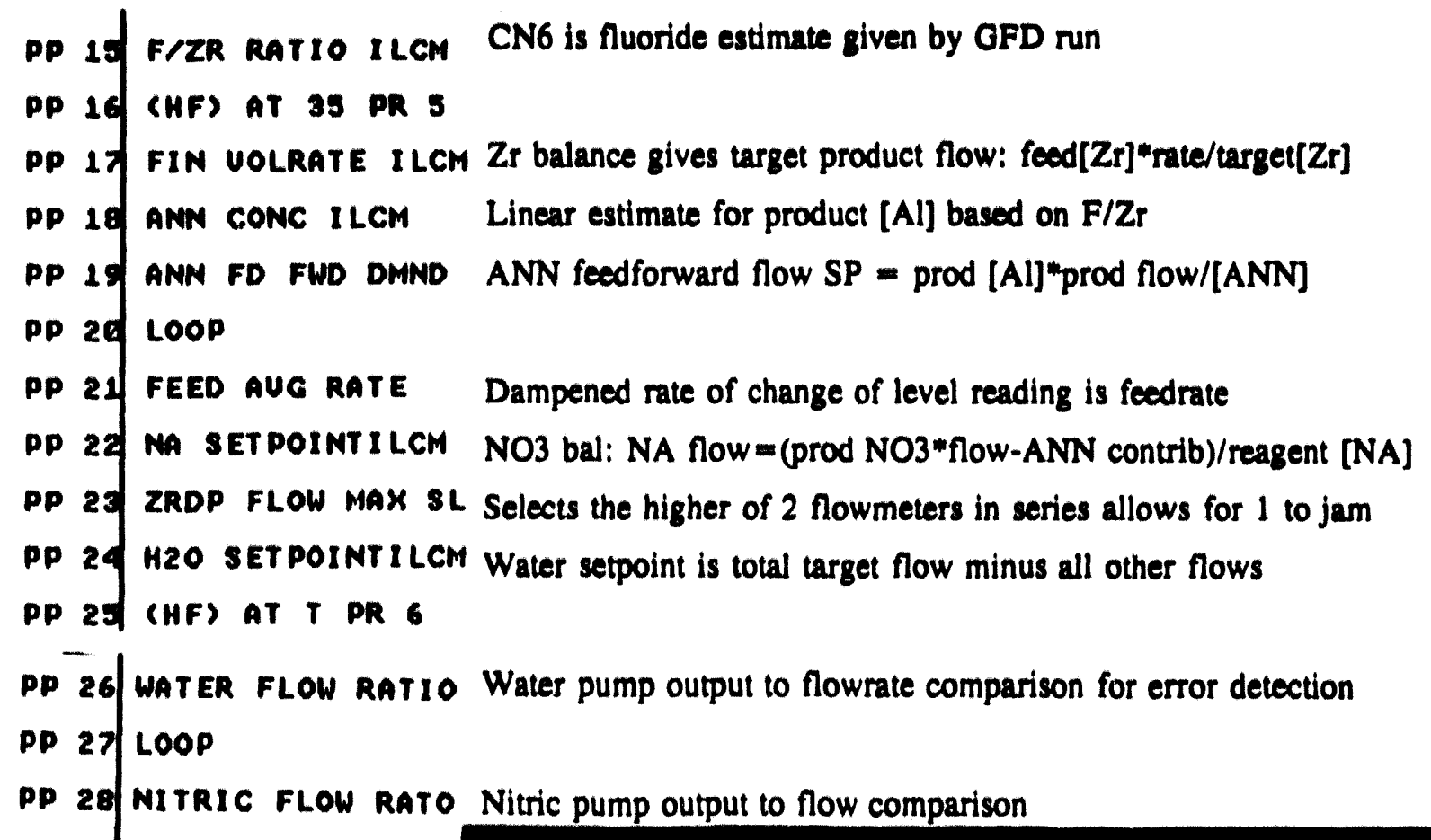




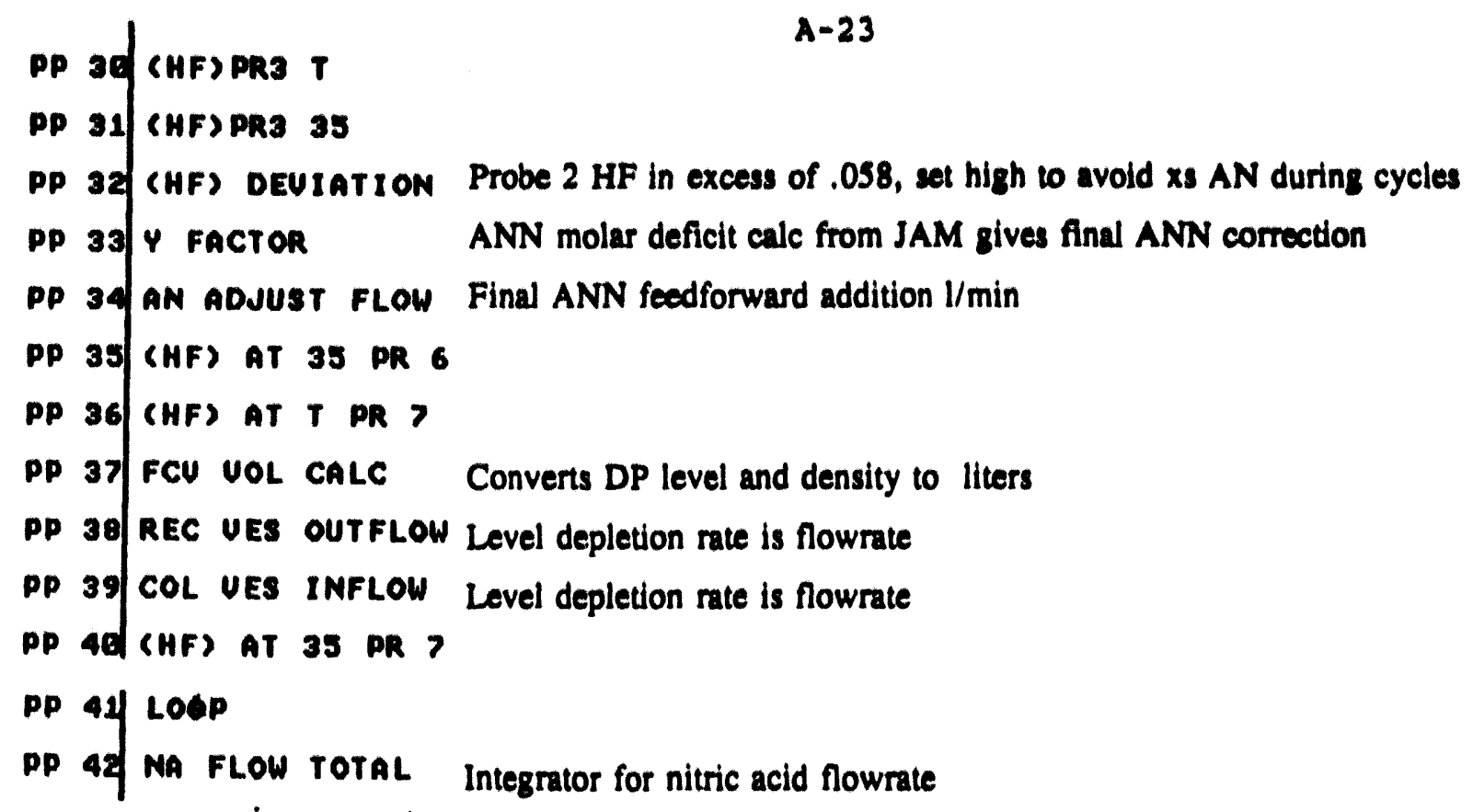

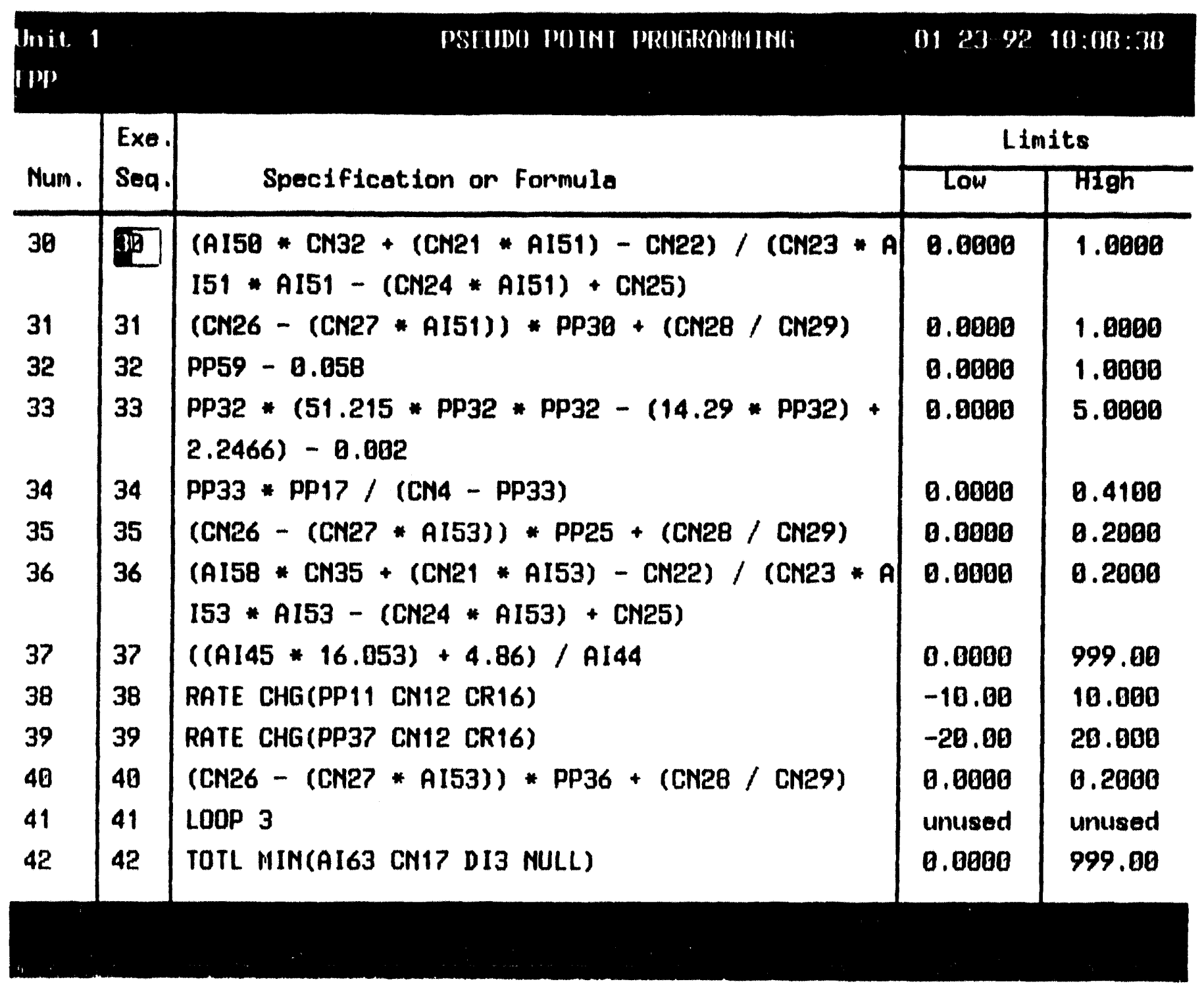


PP af WATER FLOW TOTAL Integntor for watar flow

$p$

\section{ma} ANW FLOW Tor Integrator for ANN flow

PP as AMN ADJ FLOW TOT Integrator for fina ANN flow

PP AC LP2 ADAPTIUE RST Flow proportional reset value for loop 2

PP AT PRL HF T

PP Ae PRL HF as

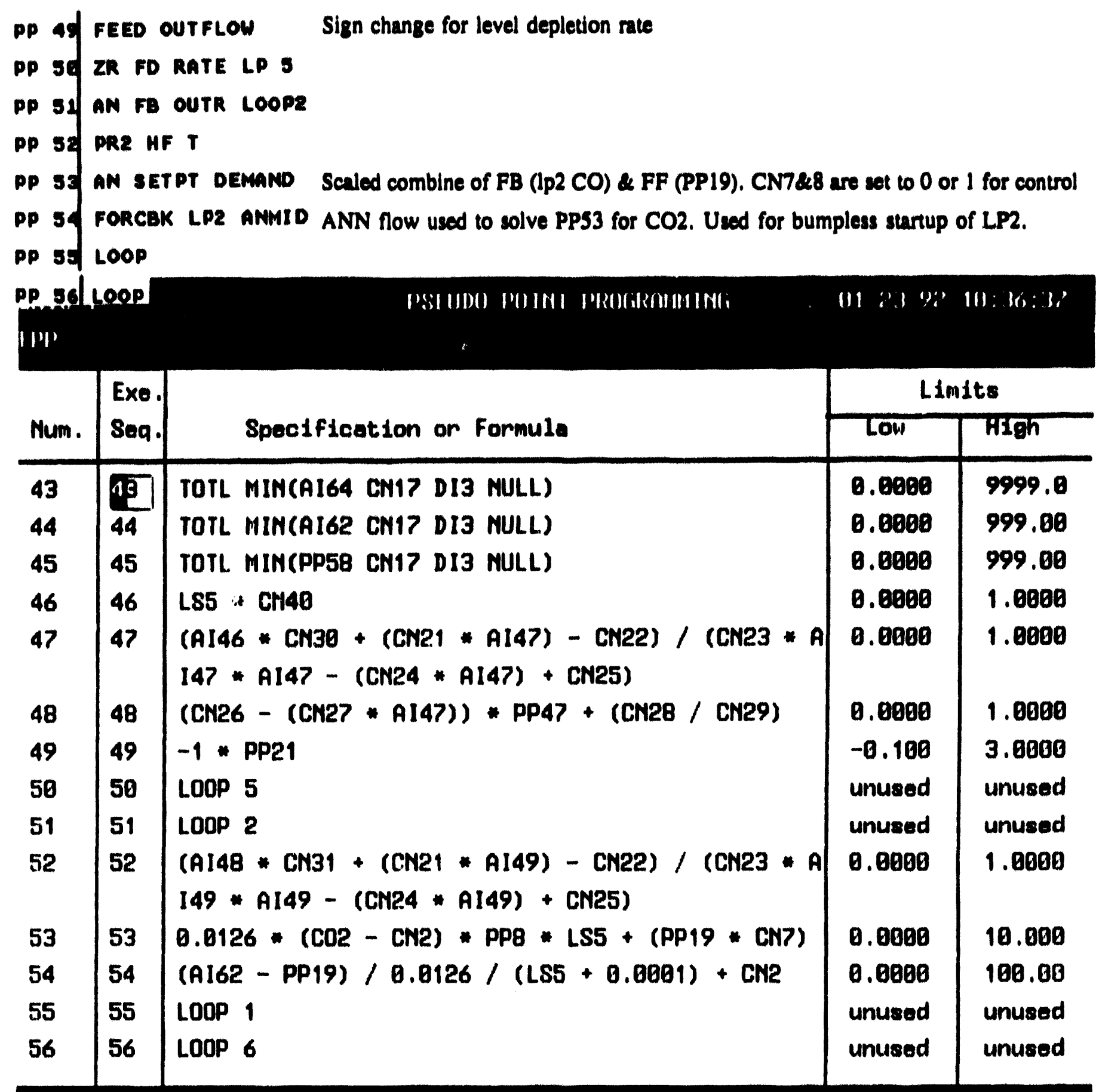




\section{$A-25$}

Pp sy Foncak LPA anout Solving for loop 4 inital output for bumpless cascade startup

pp sef Fan $x$ pump outpr sealed flow dignal for find ANN how pump output 0.100

PP S9 PR2 HF 35

PP Ge FEED FLOW TOTAL Integntor for feed flowrate

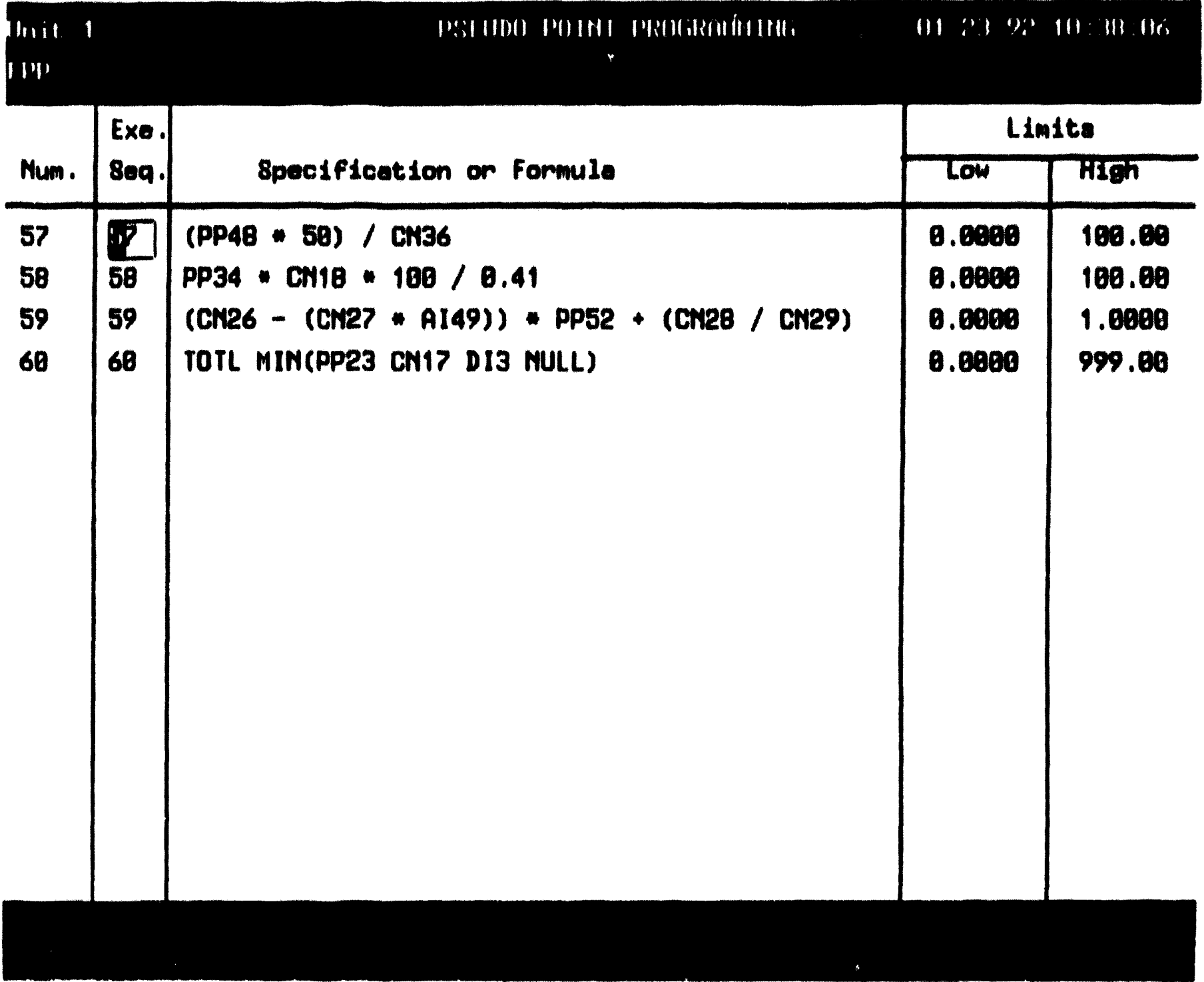




\begin{tabular}{|c|c|c|c|c|c|}
\hline $\begin{array}{ll}|1+1| 1 & 1 \\
\text { III } & \end{array}$ & & lomen:intr & IIIRY' IIIIII I & 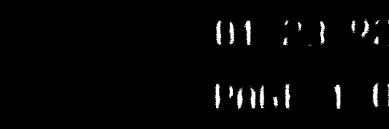 & $\because 111.18$ \\
\hline $\begin{array}{l}\text { Constant } \\
\text { Number }\end{array}$ & Desoription & Value & $\begin{array}{c}\text { Constant } \\
\text { number }\end{array}$ & Desoription & Valwe \\
\hline 1 & RECIRC \& FLON & 3.686 & 17 & FLO TOT RBET & 999.69 \\
\hline 2 & AWm LOOP 2 BIASX & 50.080 & 18 & FIN AN FF FACTOR & 1.6800 \\
\hline 3 & ZR M TARGET & 0.5800 & 19 & ASSIEN SPG VALUE & 1.2680 \\
\hline 4 & AWW M REAGENT & 2.2380 & 20 & & 3.2980 \\
\hline 5 & NA M REAGENT & 13.000 & 21 & PR & 0.6887 \\
\hline 6 & HF conc $M$ & 9.5200 & 22 & PR & 0.1280 \\
\hline 7 & AW FEED FWD FCTR & 1.0000 & 23 & PR & 0.0851 \\
\hline $\mathbf{B}$ & & 0.8880 & 24 & PR & 2.0330 \\
\hline 9 & & 3.2860 & 25 & PR & 32.540 \\
\hline 10 & 8LOPE .045 HF & 0.2476 & 26 & PR & 1.4934 \\
\hline 11 & INTERCPT .045 HF & 1.0999 & 27 & PR & 0.0143 \\
\hline 12 & FLOWRATE TIM CON & 0.2580 & 28 & PR & 0.4468 \\
\hline 13 & ZERO & 0.0800 & 29 & PR & 1080.0 \\
\hline 14 & IOME & 1.0800 & 38 & F CAL FCTR PRB I & 2.9637 \\
\hline 15 & TwO & 2.0000 & 31 & F CAL FCTR PRB 2 & 3.0880 \\
\hline 16 & THREE & 3.0080 & 32 & F CAL FCTR PRB 3 & 3.5960 \\
\hline
\end{tabular}




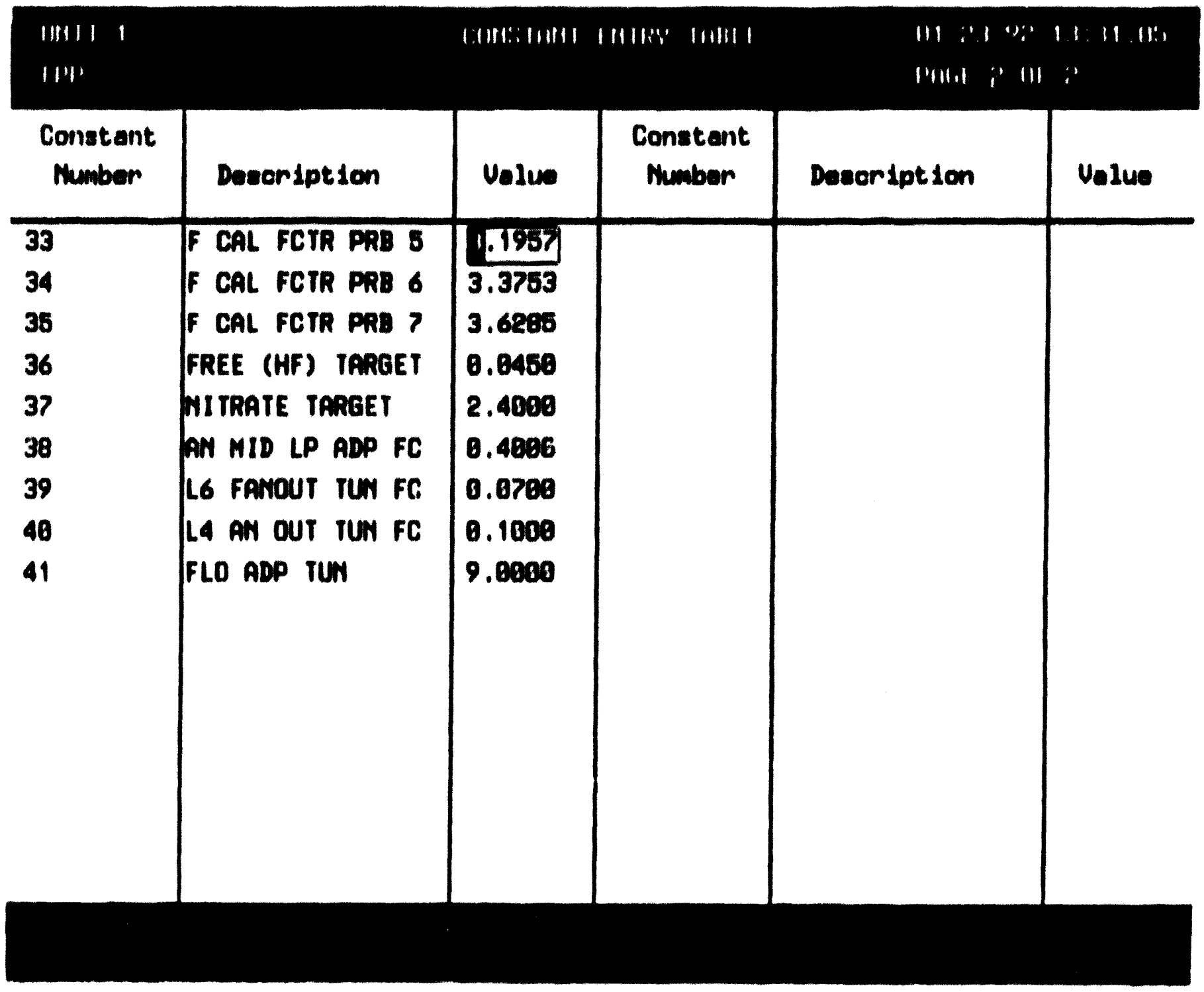


PIo conirial Loops

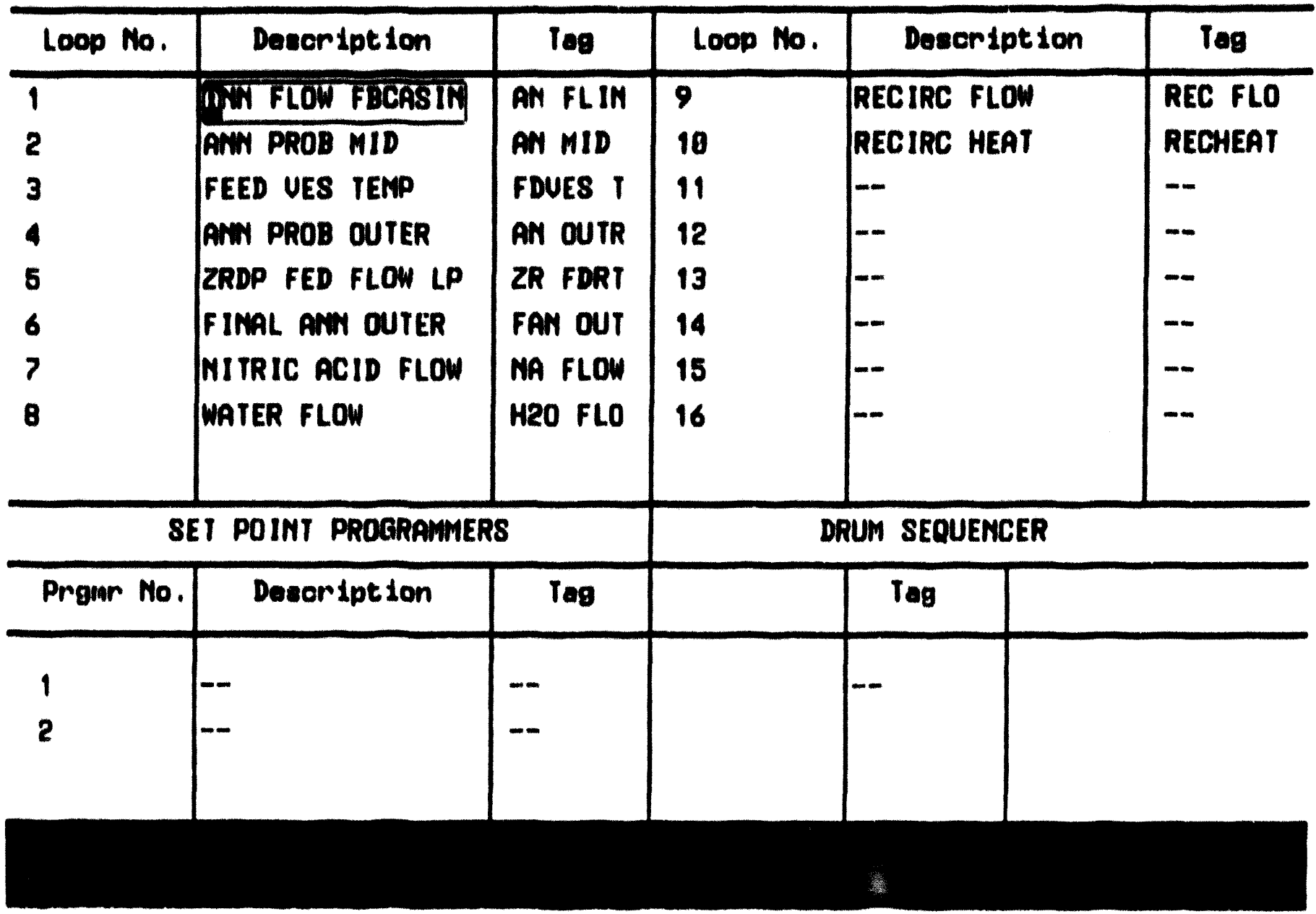




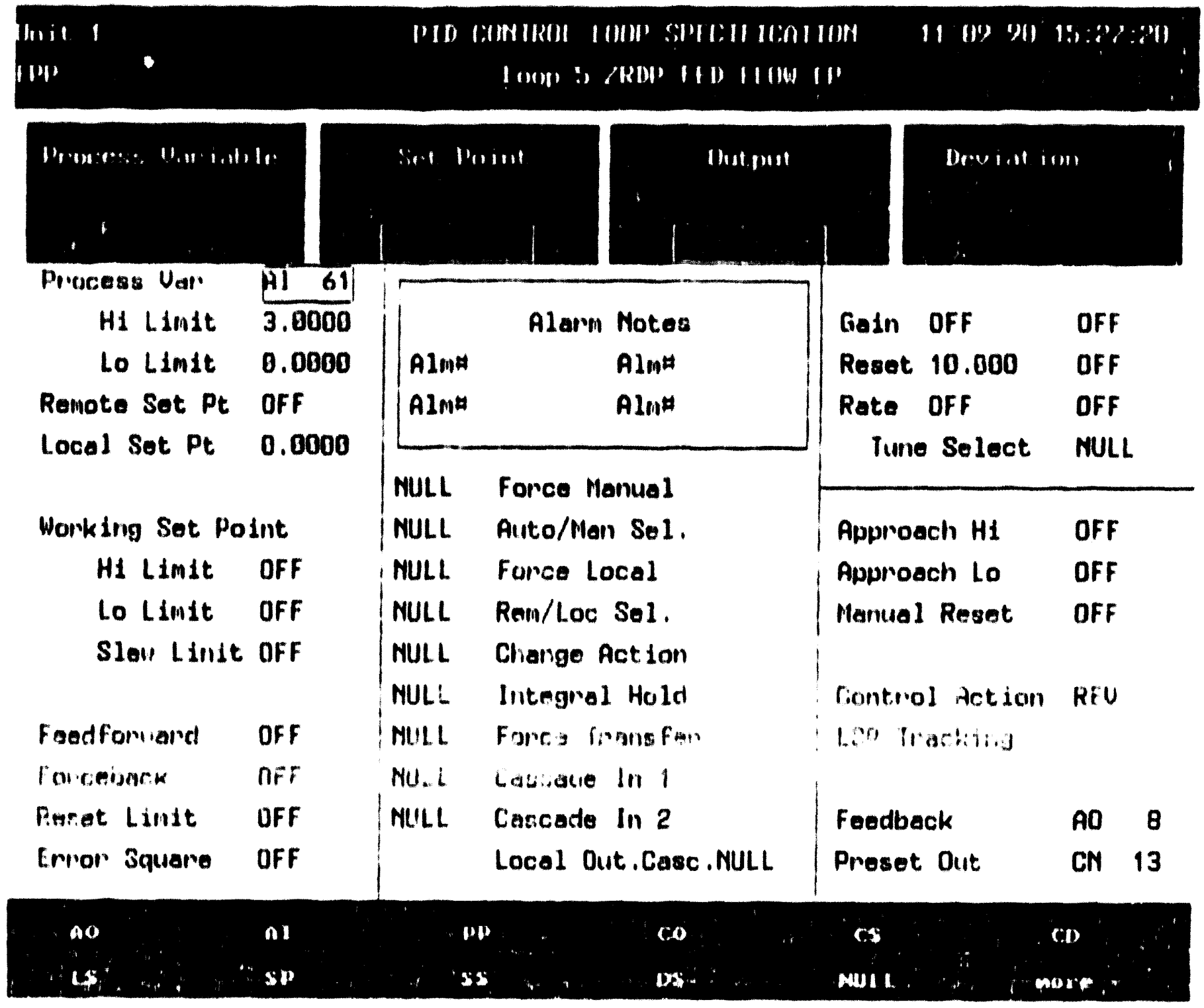




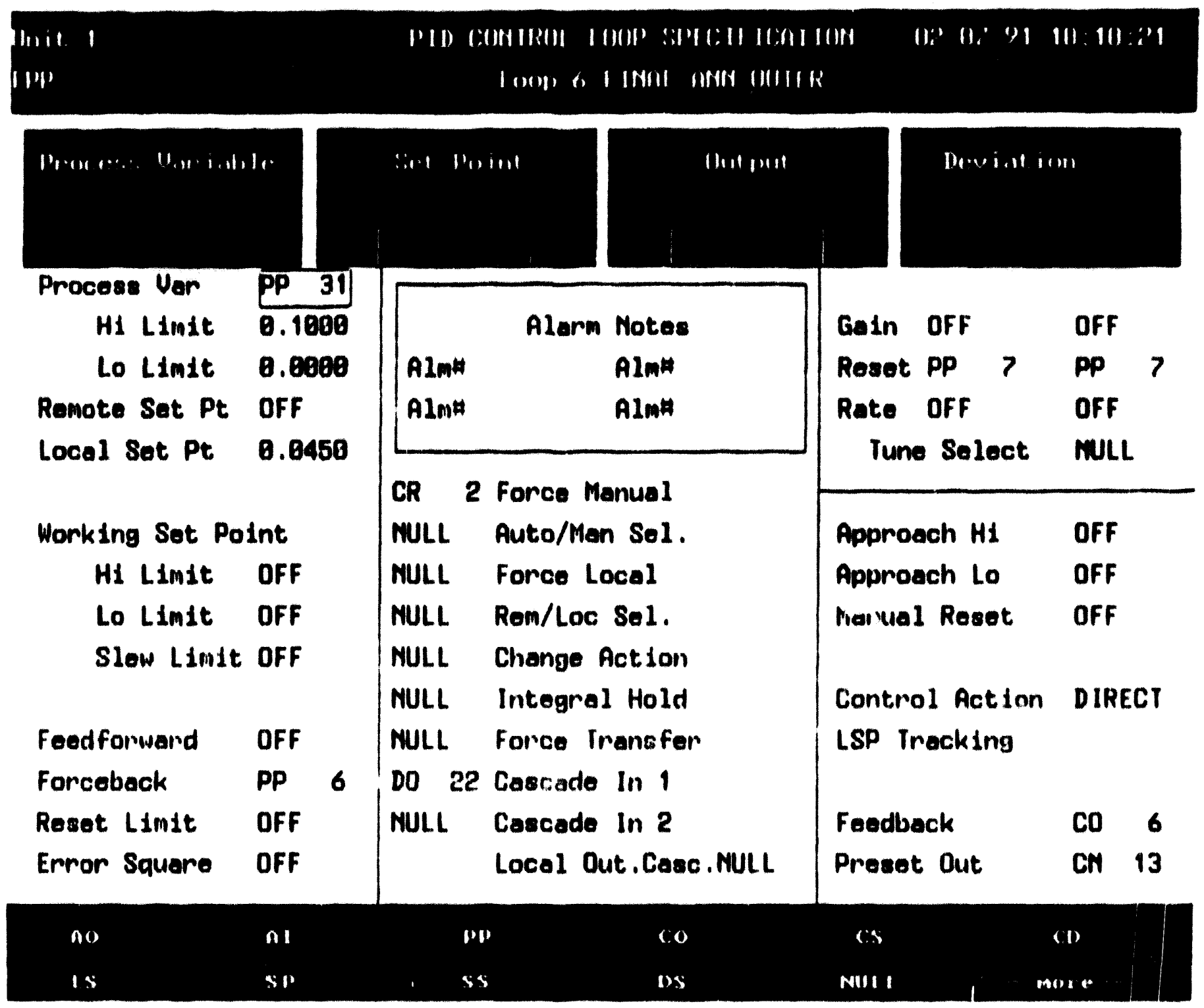




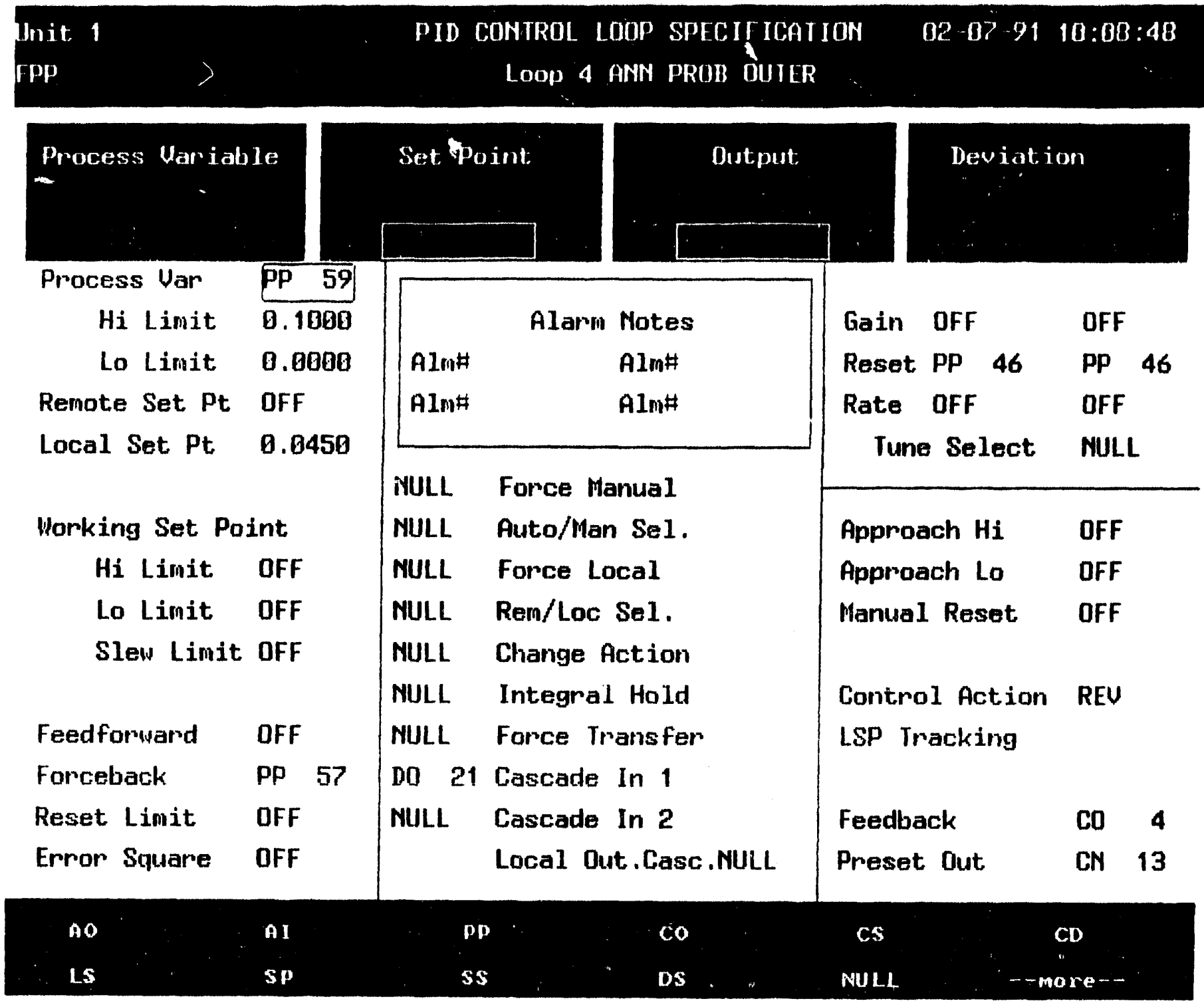




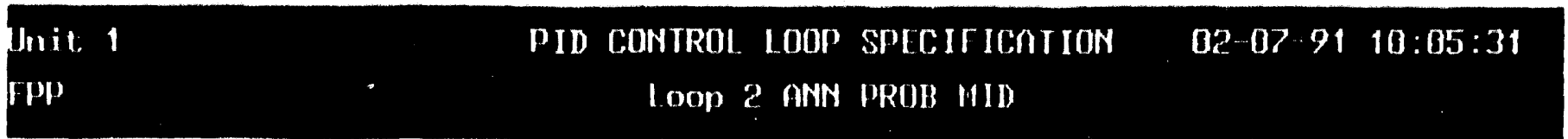

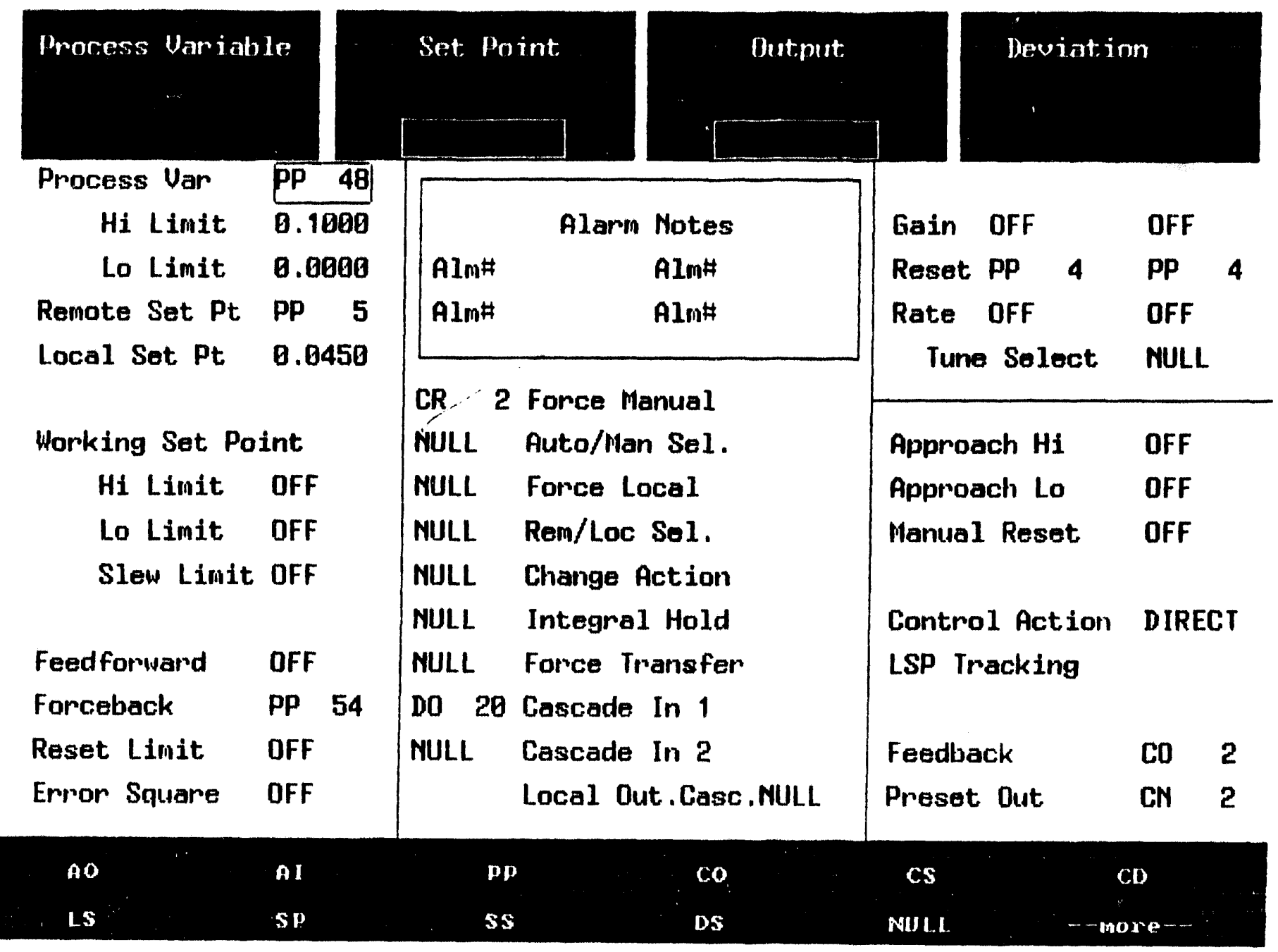




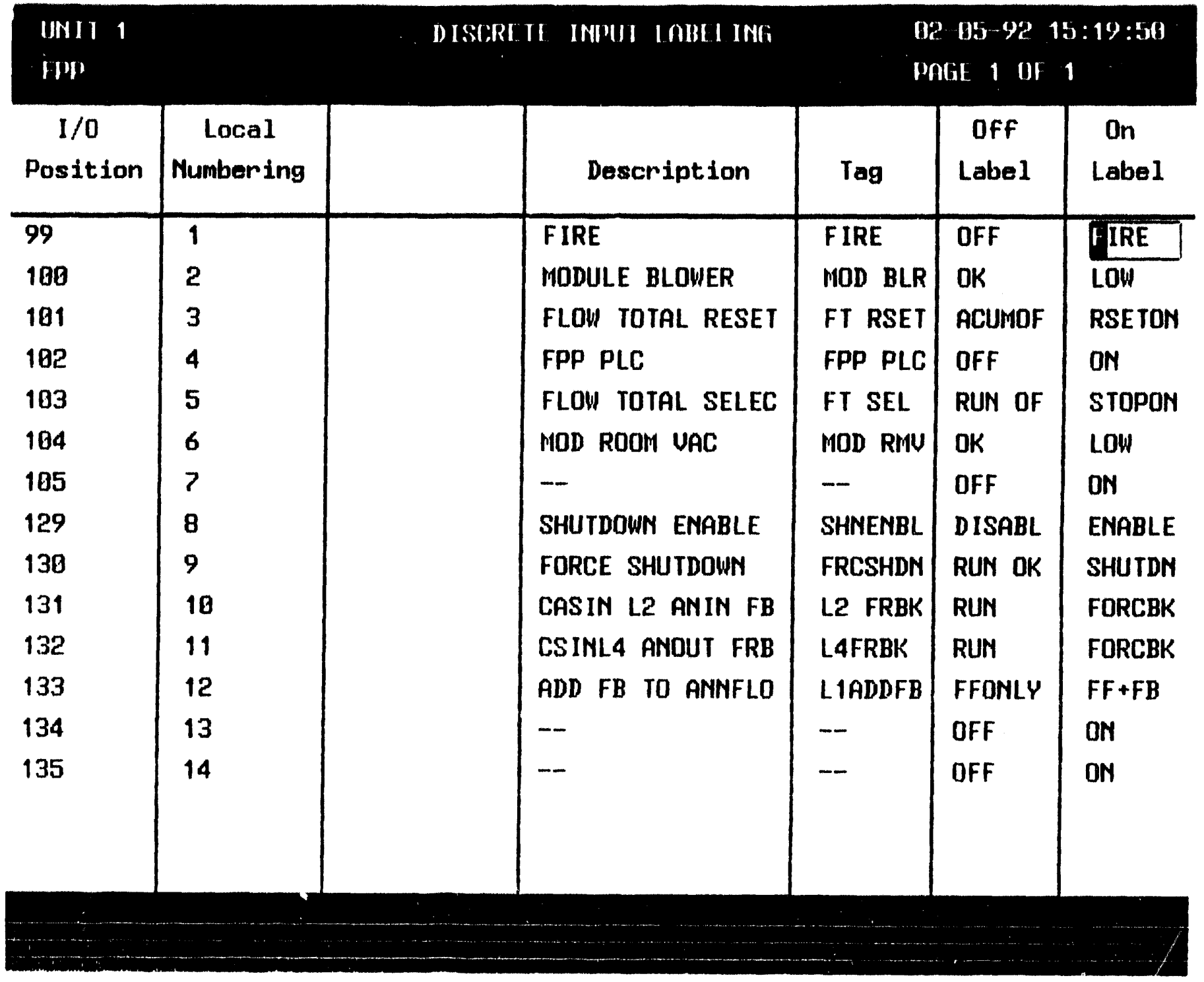




\begin{tabular}{|c|c|c|c|c|c|}
\hline $\begin{array}{l}\text { UNII } 1 \\
\text { HN }\end{array}$ & & DISERI II IIIIPUII I ABH I ING & \multicolumn{3}{|c|}{$\begin{array}{l}01: 33 \text { ga } 15: 19: 003 \\
\text { Patit } 101 \quad 3\end{array}$} \\
\hline $\begin{array}{c}1 / 0 \\
\text { Position }\end{array}$ & $\begin{array}{c}\text { Local } \\
\text { Numbaring }\end{array}$ & Description & Tay & $\begin{array}{c}\text { Off } \\
\text { Label }\end{array}$ & $\begin{array}{c}\text { On } \\
\text { Label }\end{array}$ \\
\hline 76 & 1 & & & & \\
\hline 77 & 2 & & & & \\
\hline 78 & 3 & & & & \\
\hline 79 & 4 & & & & \\
\hline 80 & 5 & & & & \\
\hline 81 & 6 & & & & \\
\hline 82 & 7 & & & & \\
\hline 83 & 8 & & & & \\
\hline 84 & 9 & & & & \\
\hline 85 & 10 & & & & \\
\hline 91 & 11 & & & & \\
\hline 92 & 12 & & & & \\
\hline 93 & 13 & FDM ANN DUMP & FDN ANN & OPEN & CLOSED \\
\hline 94 & 14 & ILCM STEAM DAMP & ICM STM & STMOFF & STM ON \\
\hline 95 & 15 & ILCM PUMP CTRL & ICMPUMP & AUT OF & MAN ON \\
\hline 96 & 16 & ILCM FIN ANN CTL & FIN ANM & AUT OF & MAN ON \\
\hline
\end{tabular}




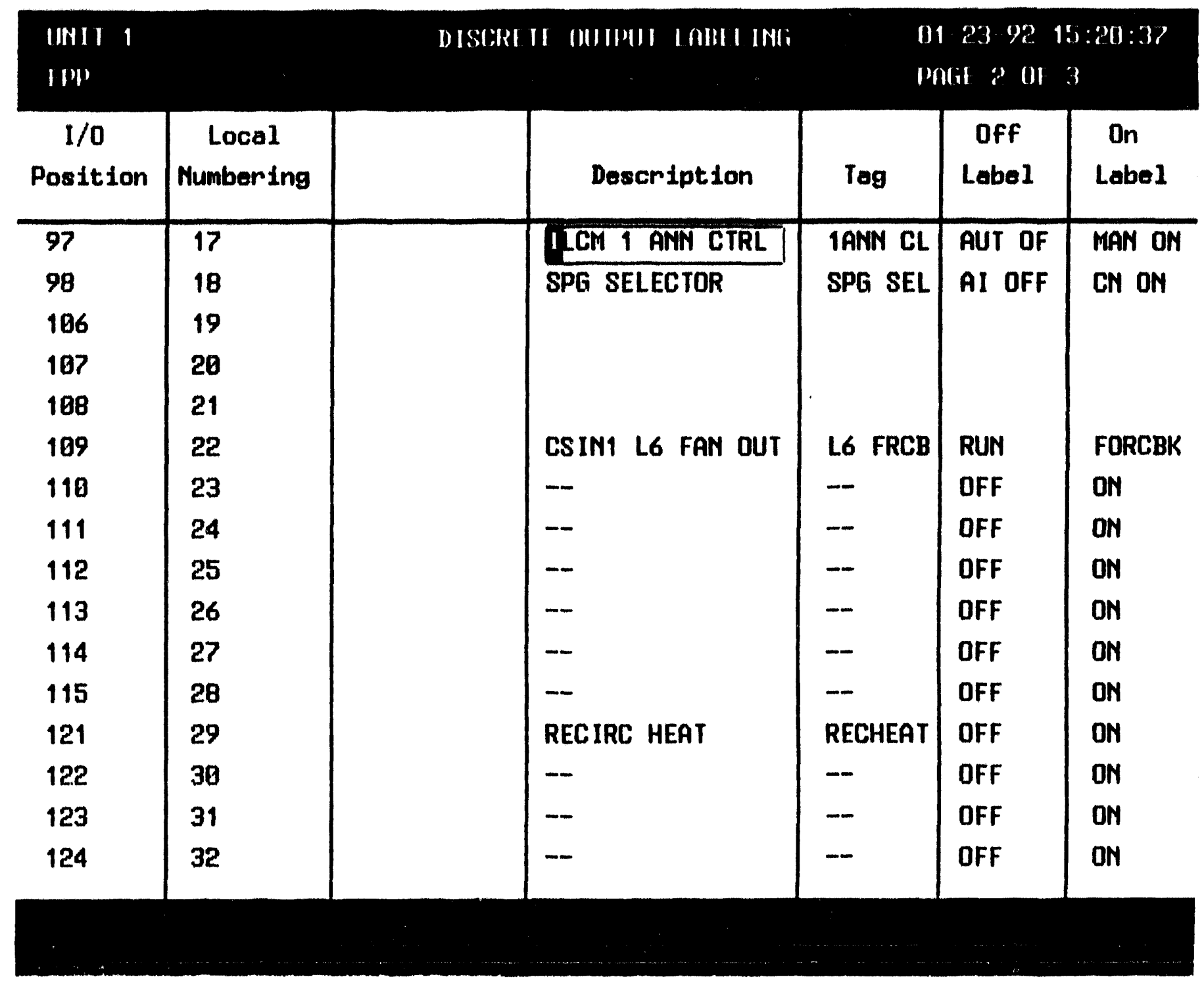




\begin{tabular}{|c|c|c|c|c|c|c|c|}
\hline $\begin{array}{l}\text { Init. } \\
\text { INI }\end{array}$ & & DONIROI RII AY ASS & & & $\begin{array}{ll}11 & 19 \\
& M\end{array}$ & $\begin{array}{l}98 \\
\text { 9. }\end{array}$ & $1: 46: 39$ \\
\hline $\begin{array}{l}\text { CR } \\
\text { Mo. }\end{array}$ & Description & Assignment & $\begin{array}{l}\text { AMD } \\
\text { /OR }\end{array}$ & Alarm & Event & $\begin{array}{l}\text { Tar. } \\
\text { Ser }\end{array}$ & $\begin{array}{l}\text { Global } \\
\text { Output }\end{array}$ \\
\hline 1 & FEED FLOW DEUIAT & $\overline{A L ~} 1$ & $a r$ & & Rep & (1) & $\bar{\theta}$ \\
\hline 2 & RECYCLE OFF & PLC & Or & & Rep & $\theta$ & $\theta$ \\
\hline 3 & LO ZRDP FEED LEU & AL 4 & Or & & Rep & 0 & $\theta$ \\
\hline 4 & SHUTDOWN & PLC & Or & & Rep & $\theta$ & $\theta$ \\
\hline 5 & ANM FLOW & AL 6 & Or & & Rep & $\theta$ & $\theta$ \\
\hline 6 & ZR FEED FLOW & AL 7 & Or & & Rep & $\theta$ & $\theta$ \\
\hline 7 & WATER FLOW & AL 8 & Or & & Rep & $\theta$ & B \\
\hline 8 & MITRIC FLOW & AL 9 & Or & & Rap & 0 & 0 \\
\hline 9 & REC UES TEMP & AL 10 & Or & & Rep & $\theta$ & 0 \\
\hline 10 & REC UES LUL & AL 11 & Or & & Rep & 0 & $\theta$ \\
\hline 11 & REC UES UAC & AL 12 & Or & & Rep & 0 & $\theta$ \\
\hline 12 & LOW MOD UAC & PLC & Or & & Rep & o & $\theta$ \\
\hline 13 & LOW HEPA DP & AL 13 & $0 n$ & & Rep & 0 & $B$ \\
\hline 14 & FLO TOT SWITCH & PLC & Or & & & $\theta$ & 0 \\
\hline 15 & FCU HI LEUEL & AL 14 & On & & Rep & 0 & $B$ \\
\hline 16 & HIGH HF & AL 5 & Dn & & & 0 & $\theta$ \\
\hline
\end{tabular}




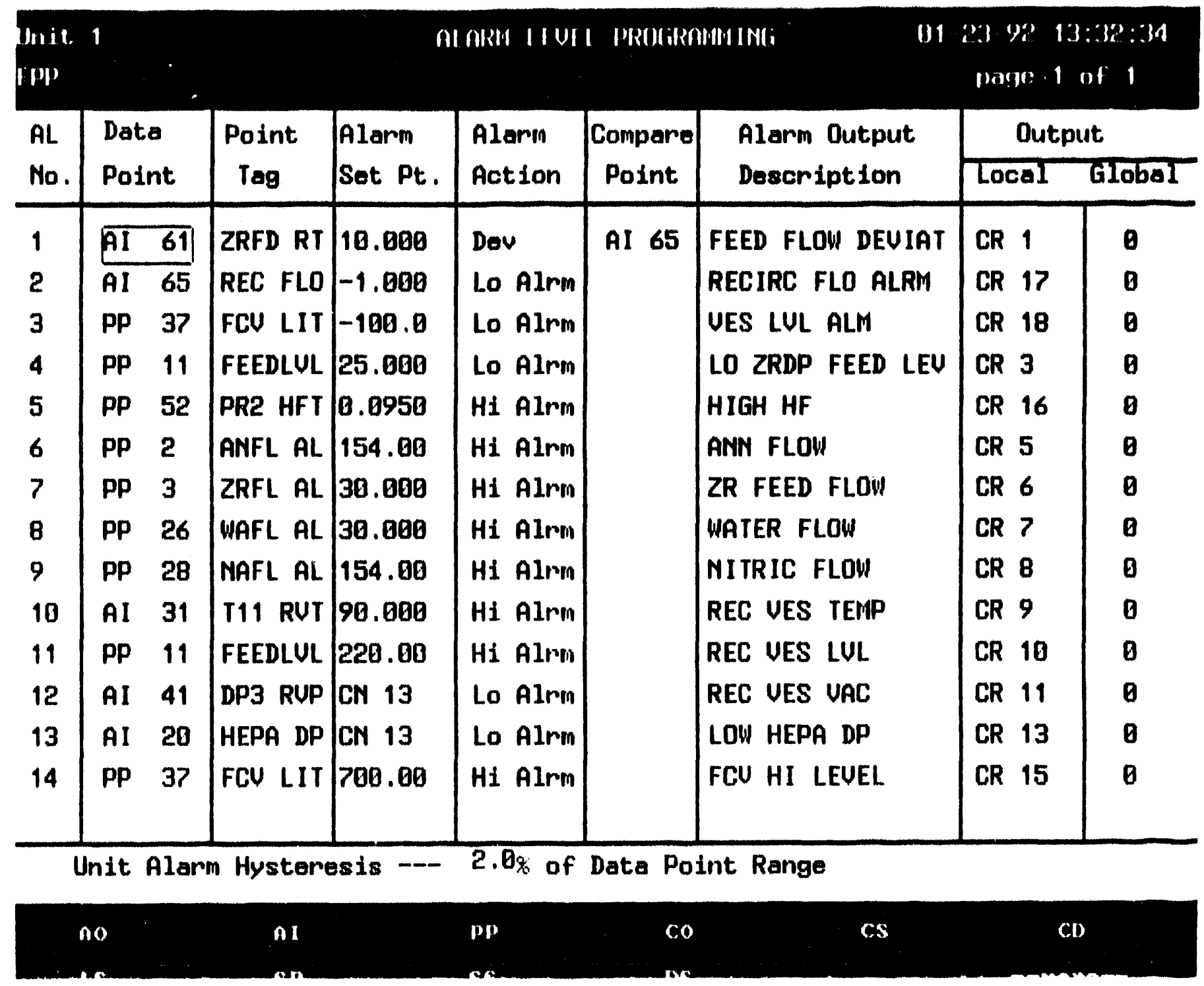


Unit 1 FPP

05i-29-91 10:11:07

PID Control Loop

5

ZRDP FED FLOW LP SAUED

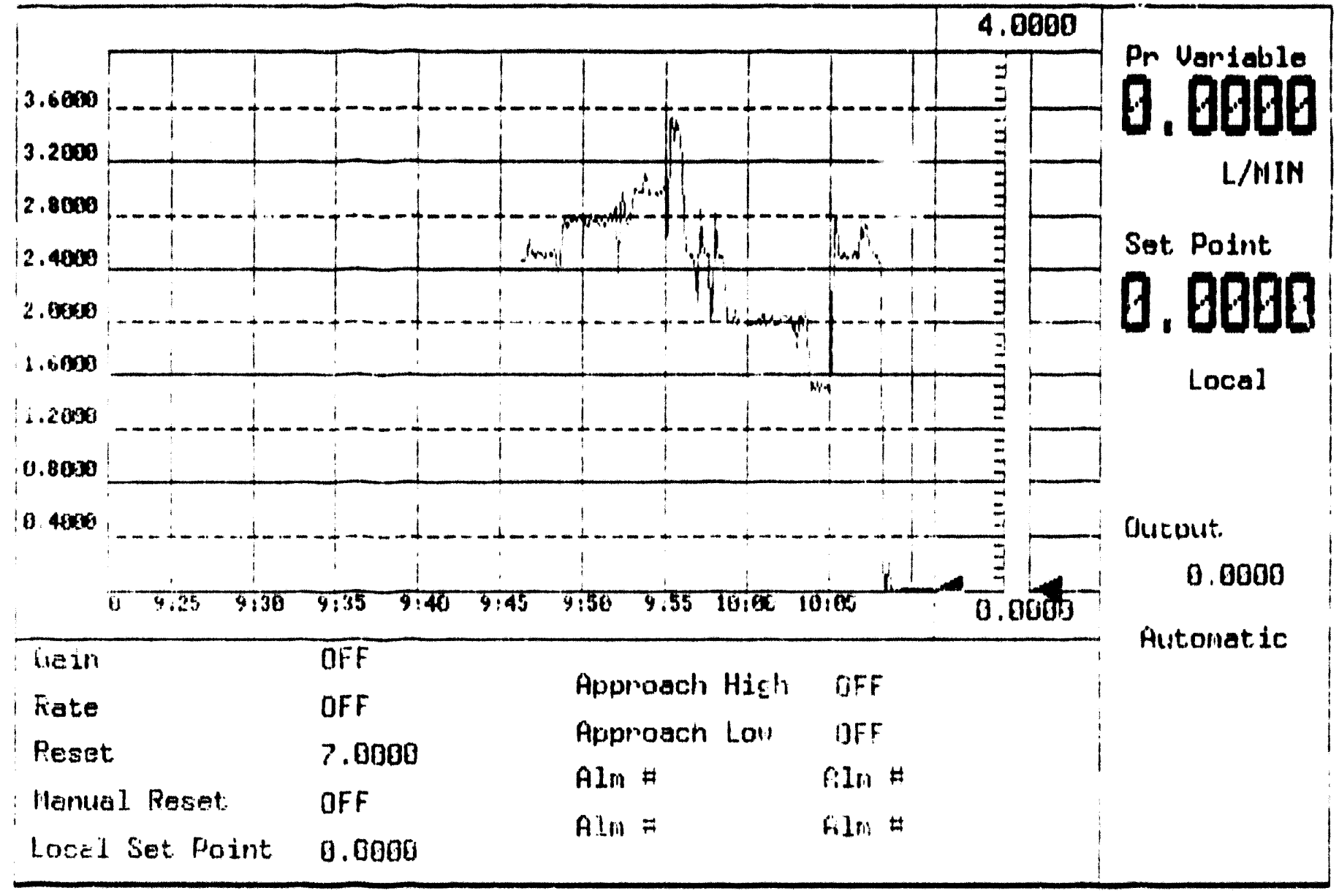


Uildt 1 FPP

PID Control Loop 5

ZRDP FED FLOW LP

$05-28-91 \quad 15: 55: 00$

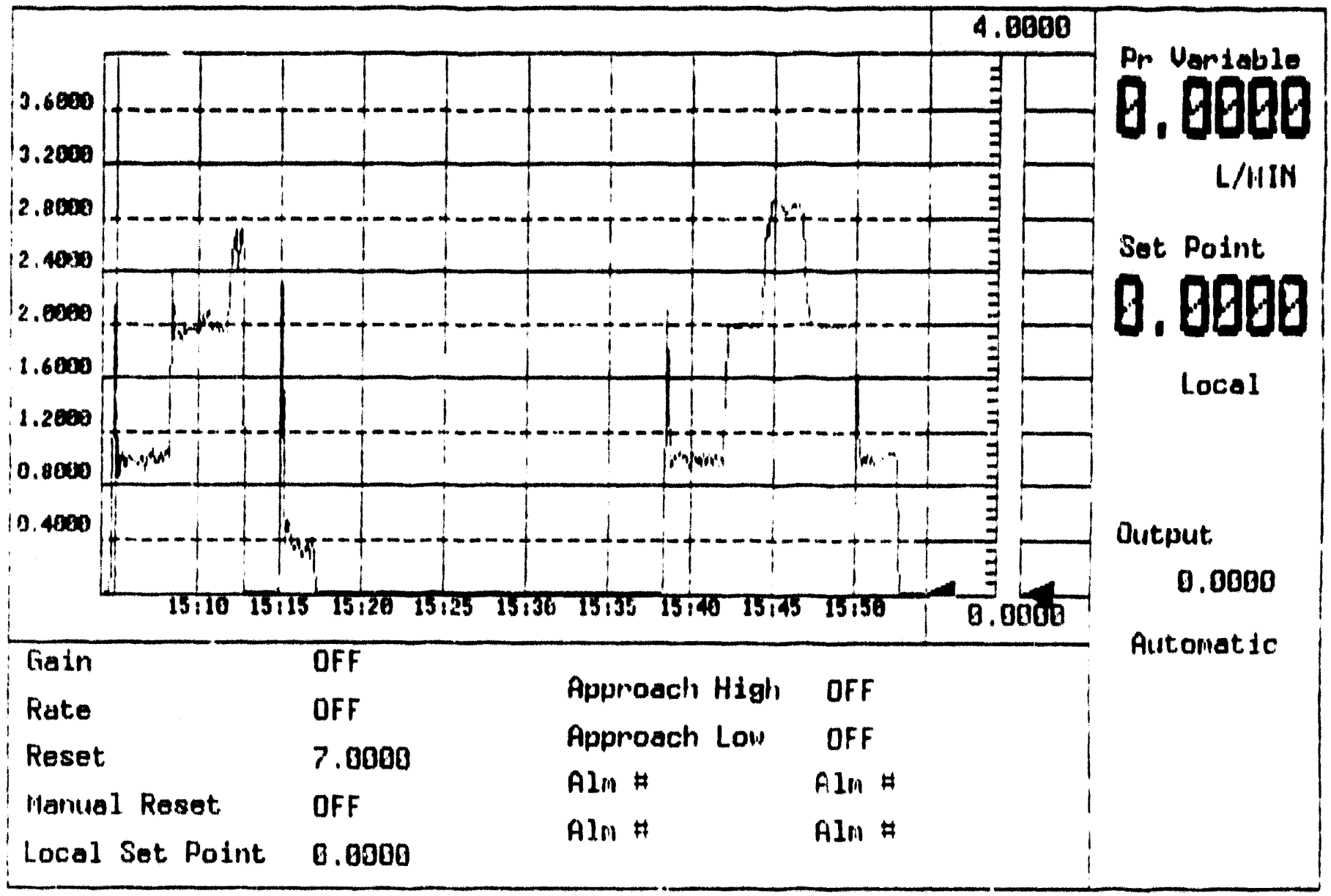


Unit 1 FPP

PID Control Loop 5

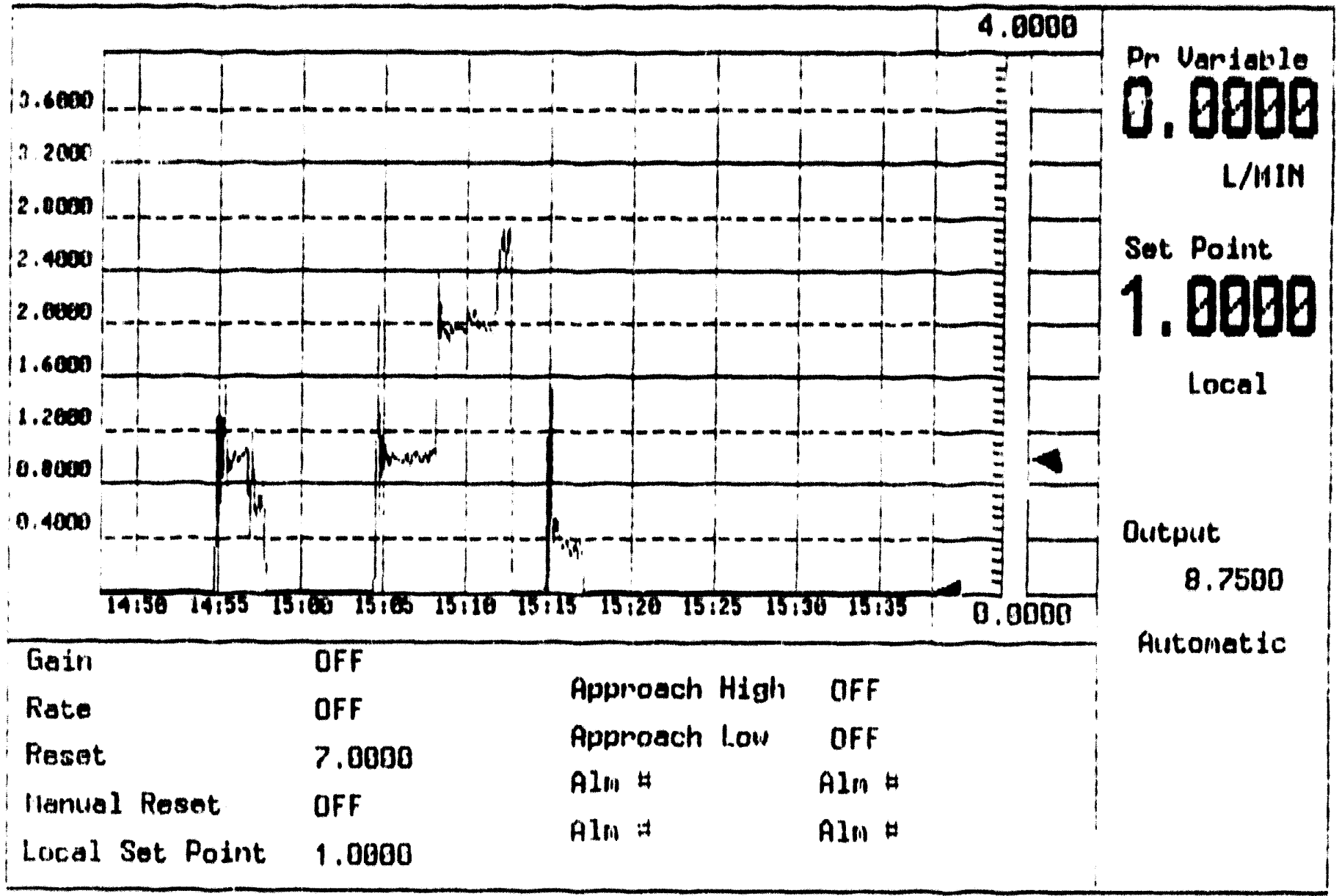


Group ?

OPER PARAMETERS

05-29-91 19:25:02

SAUED

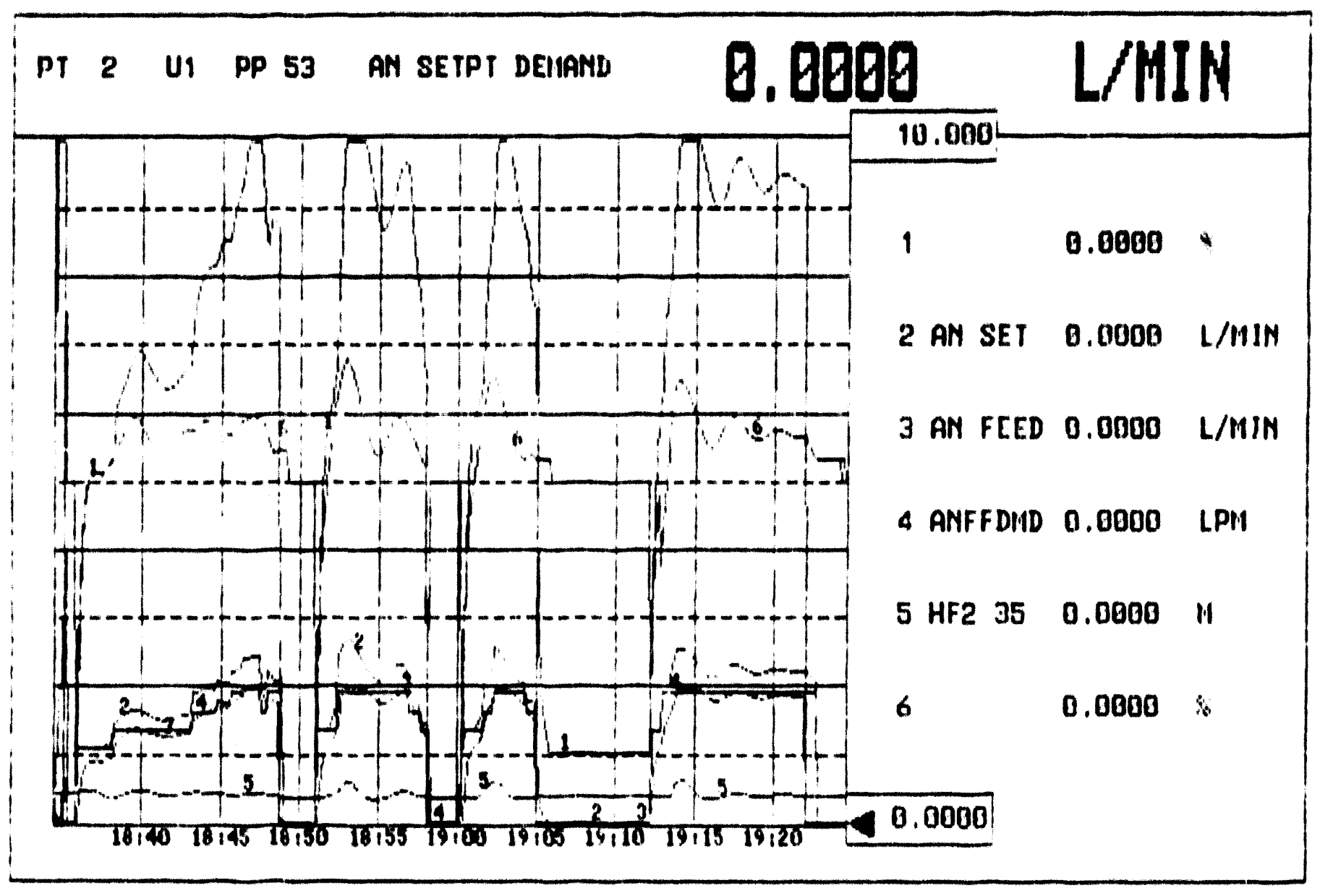


Repont 1

INL INE COMPLEK

05-29-91 19:21:41

U1 15

UI LA U1 L2

UI LI

U1 16

U1 27

U1 28

U1 13

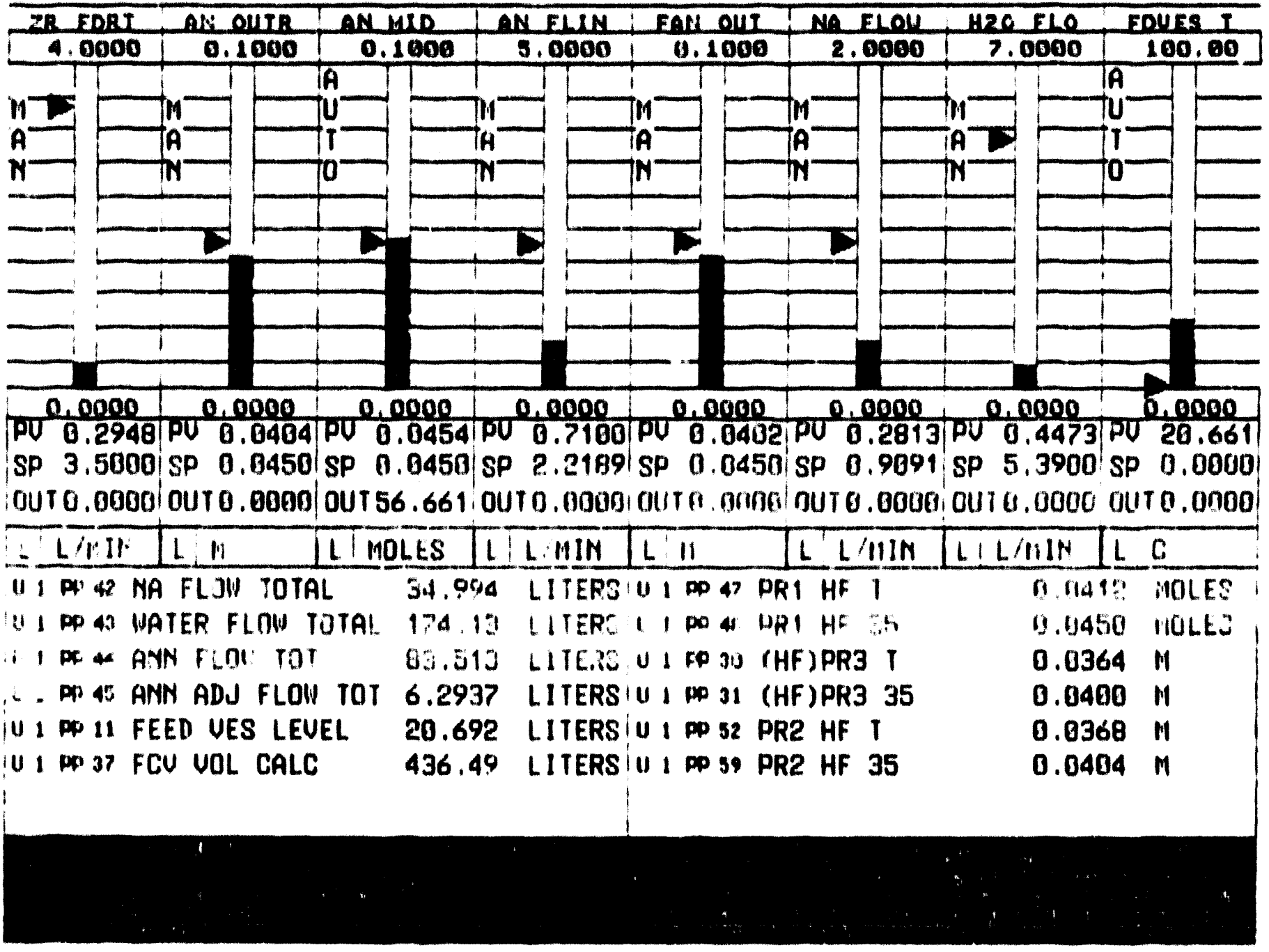


Group 3

(HF) AT 35

05-29-91 19:23:29

SAUED

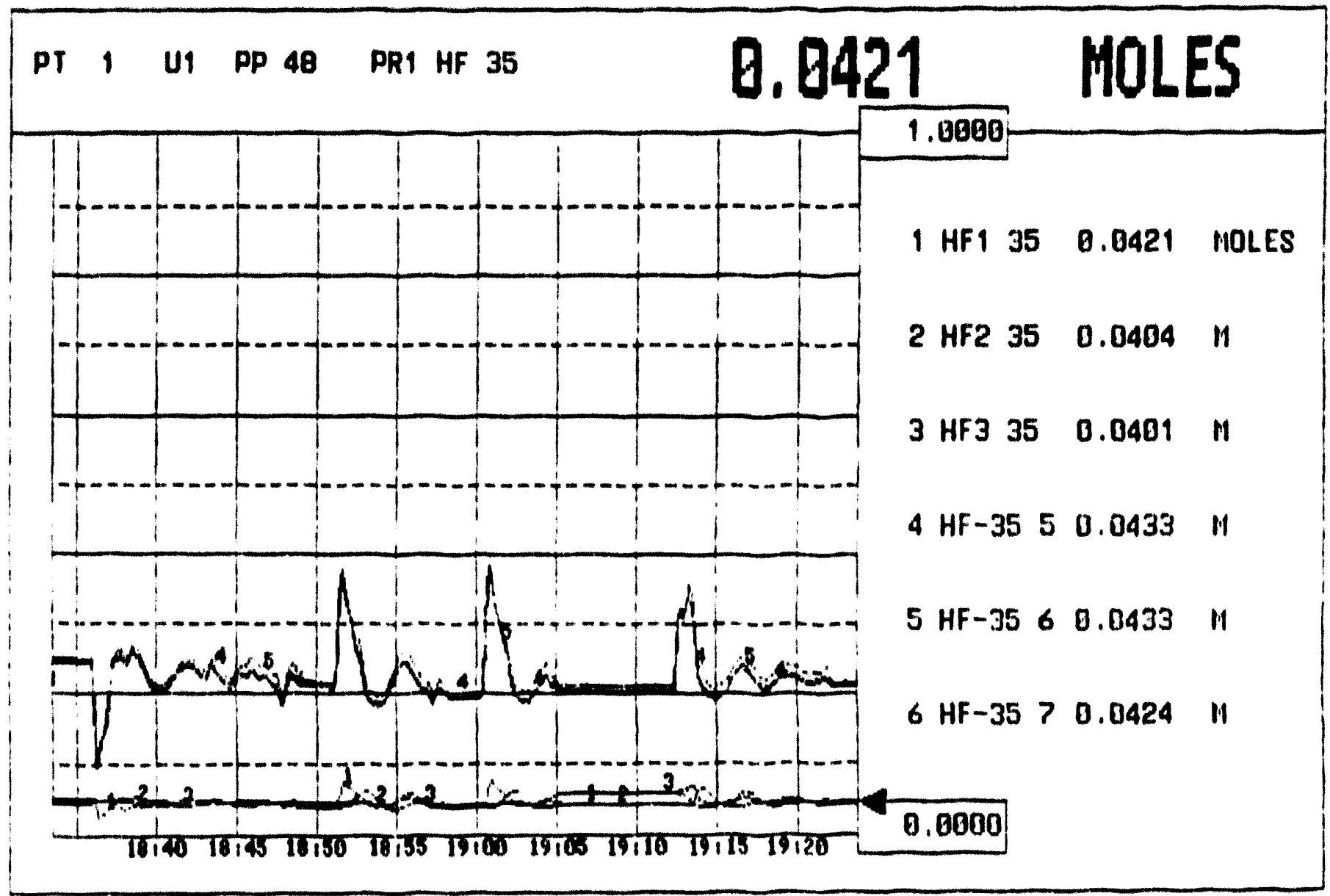




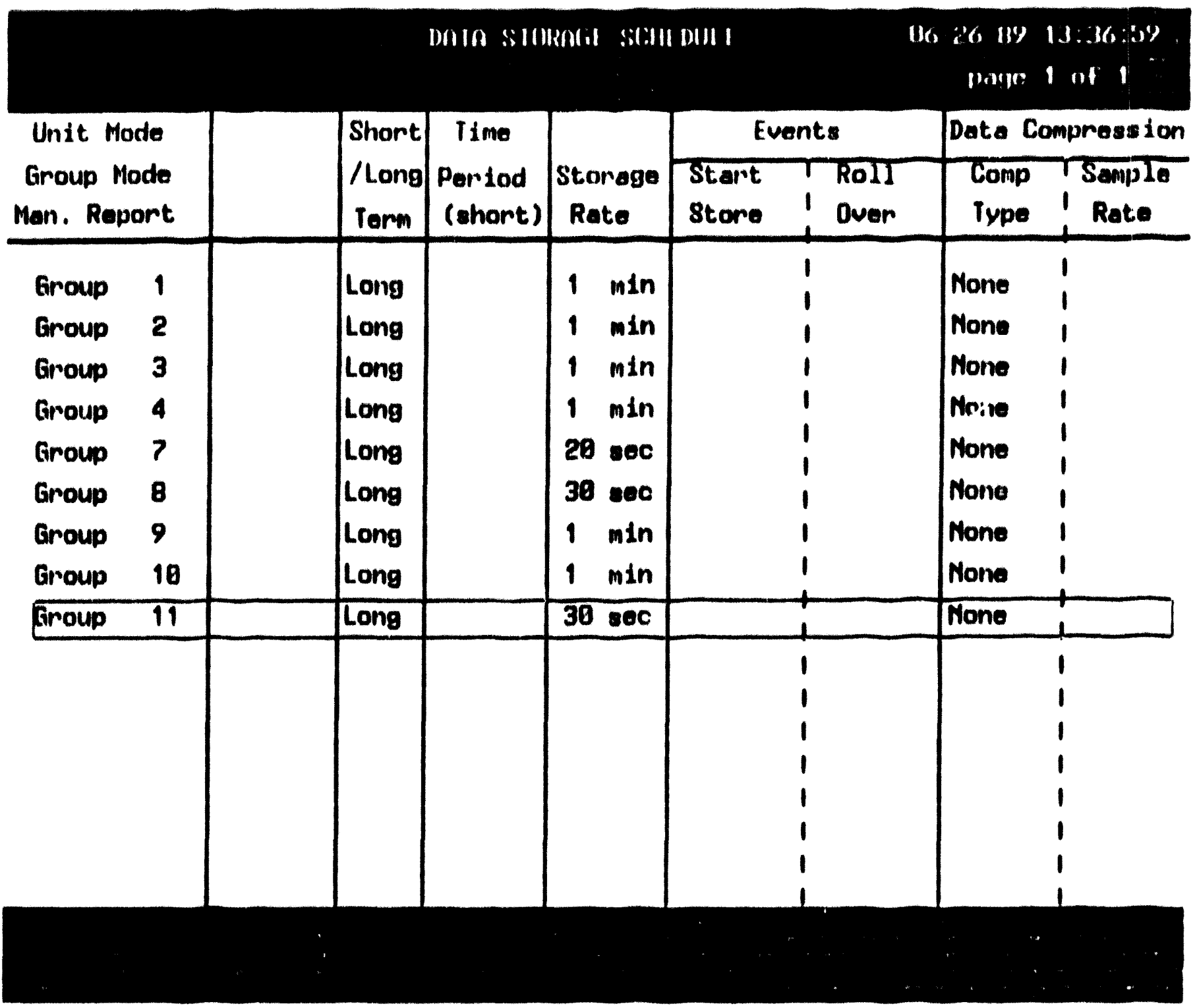




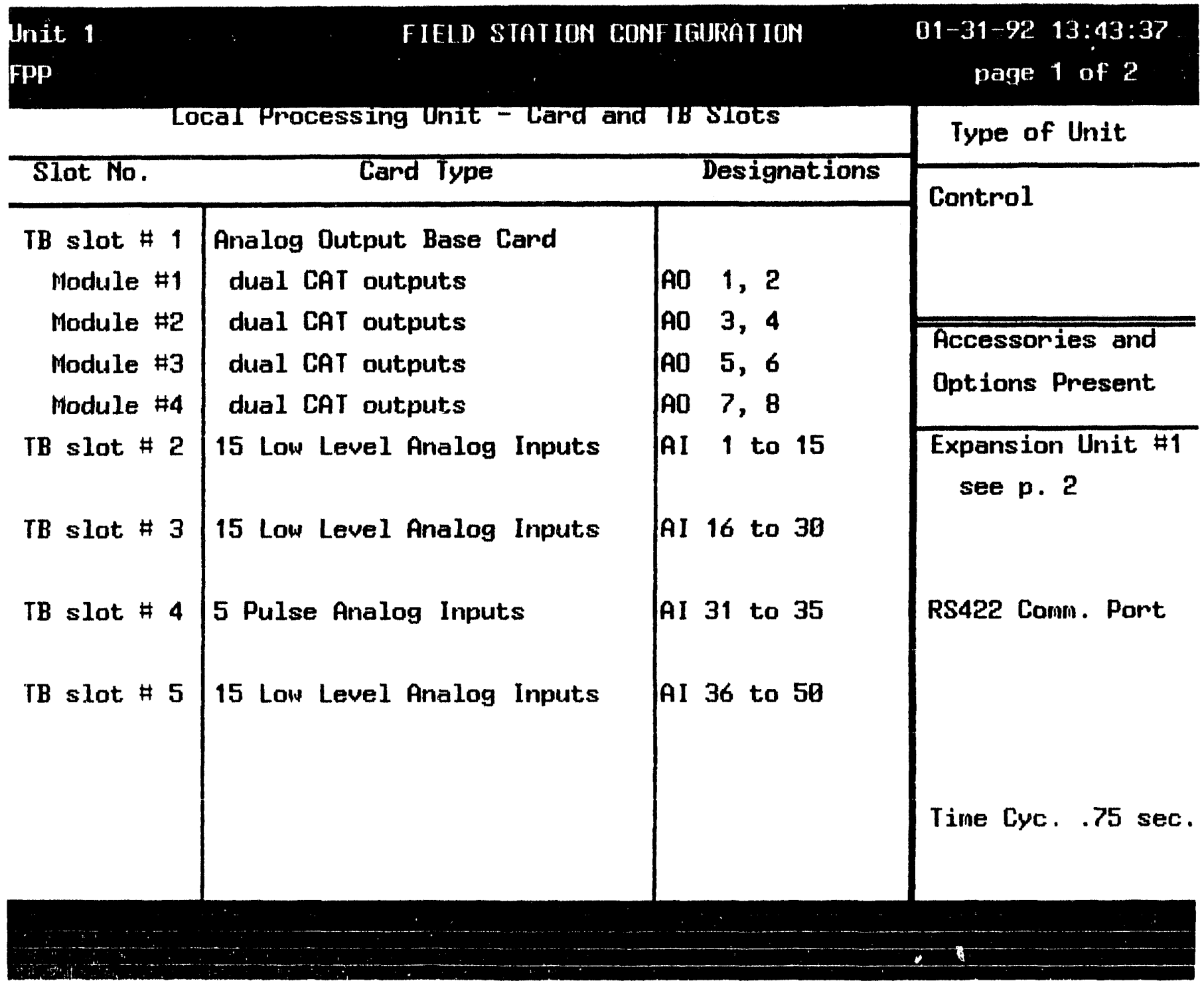




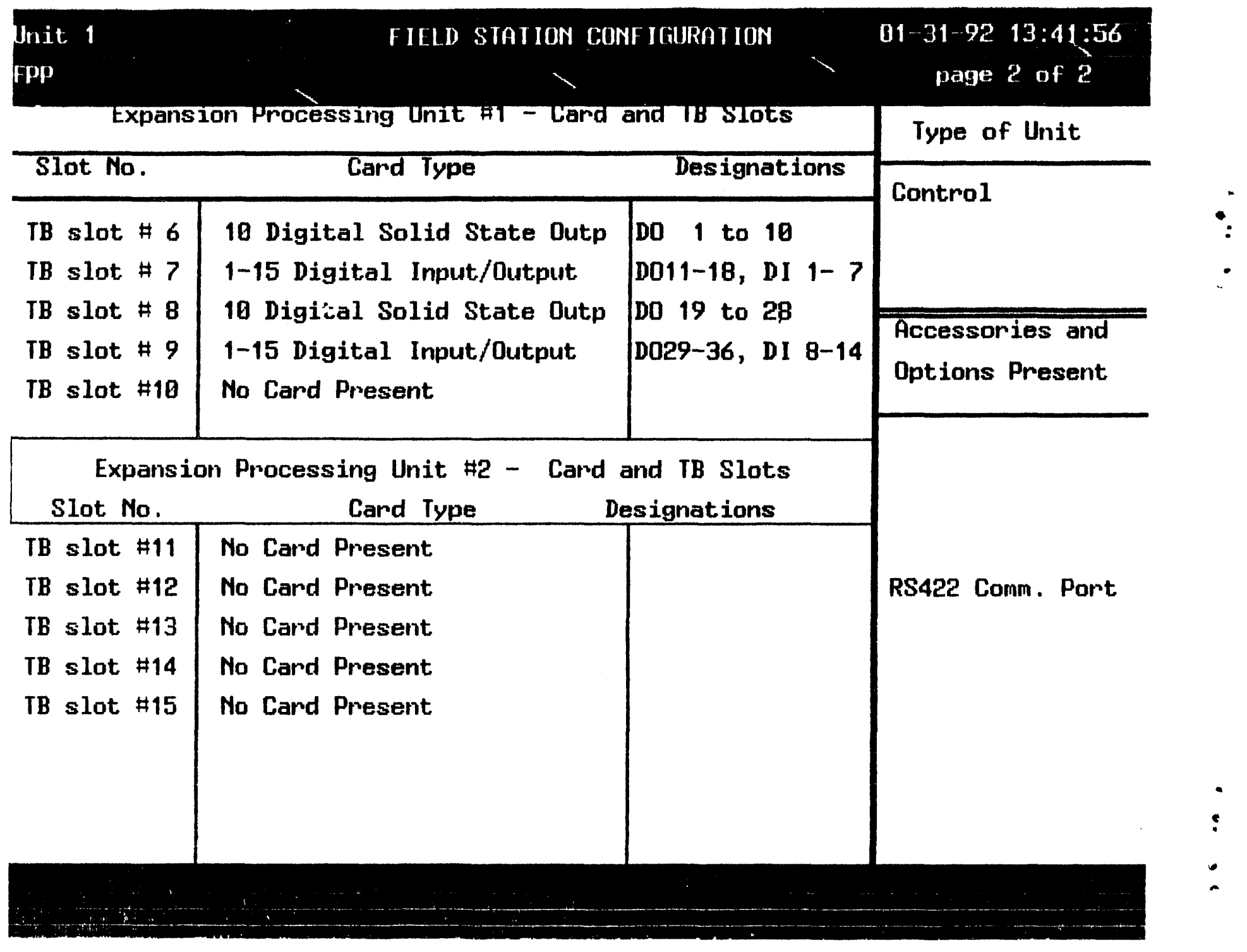



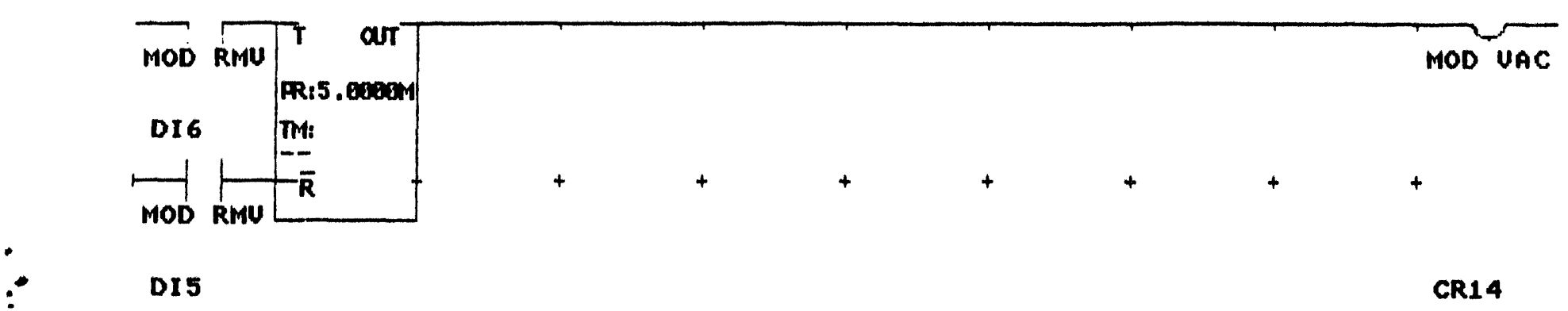

$\therefore$ DI5 CR14
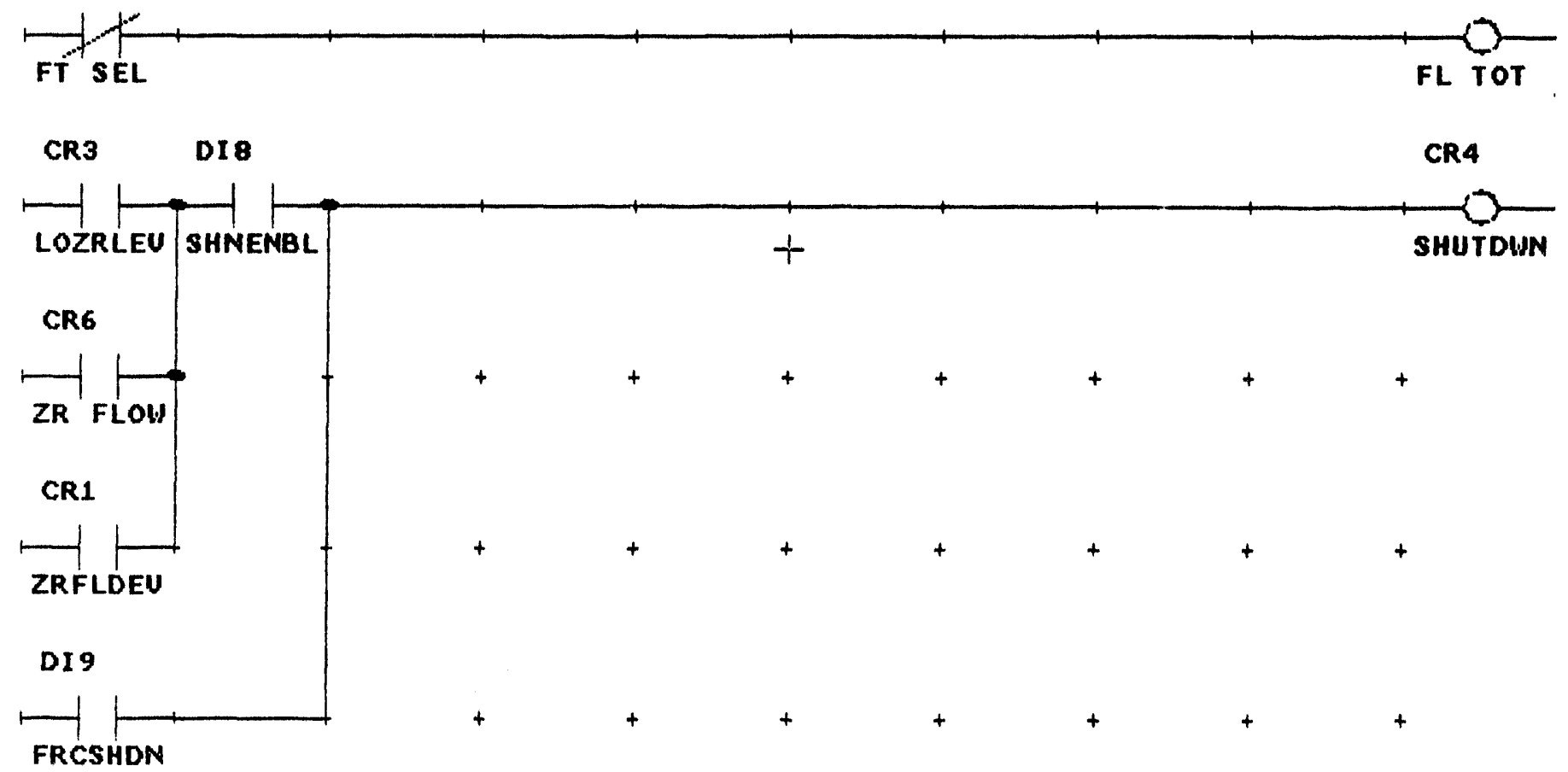

?

FRCSHDN 


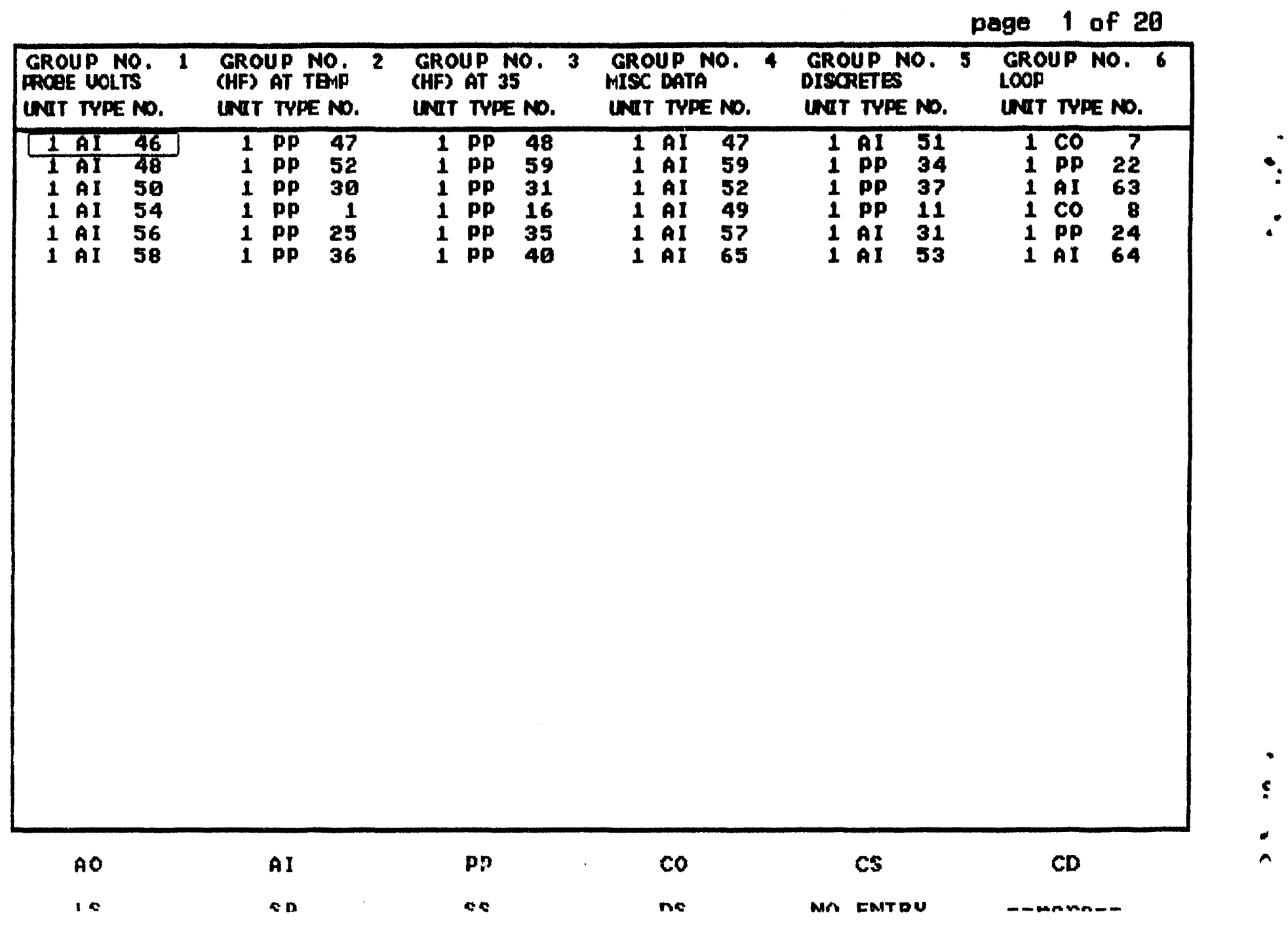




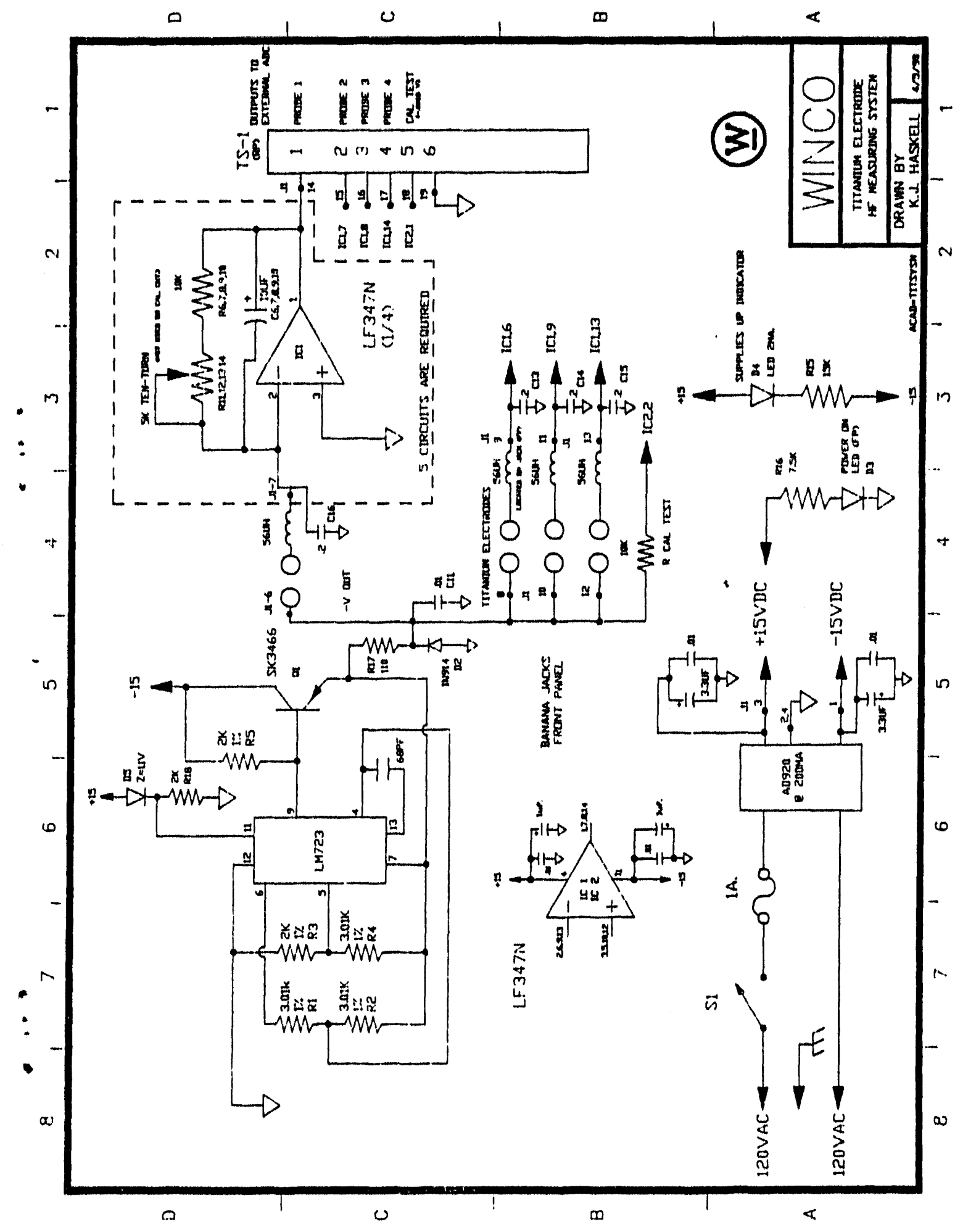




\section{ILCM PRE-RUN CHECKOUT}

Test run reagent loops on manual (15\%) for water, ANN, Nitric acid, Final ANN. Be sure valves are open. Check for flow reading on correct turbine meter. Be as brief as possible.

Check turbine meter signal voltage on feed pump. Should be 0.6 to 4.7 volts. Run feed pump (briefly) in manual (88) with valves closed to check direction of rotation.

Check TC's, tank levels, and densities for valid signals.

Check operation of probe loop recirculation pumps.

Check HF probe leads with precision resistor "probe" $2 \mathrm{~K}$ to $50 \mathrm{~K}$ ohms. Do not ground. Check for valid signals on proper channels on computer.

\section{STARTUP CHECKLIST FOR ILCM OPERATION}

Heat solution to desired temperature Connect feed flowmeter to black cable \#1 Connect TC's 1, 2, and 3 to sockets with same numbers. Calibrate Probes

Record reagent tank volumes in logbook

Set $\mathrm{HF}$ target $(\mathrm{CN} 36) \quad .045$

Set reagent concentrations for ANN (CN4) and Nitric Acid (CN5)

Set product $\mathrm{Zr}$ target (CN3)

Verify correct data tape is in DSU and storage of data to tape is allowed

Valid 25 degree C SPG (CN19), HF (CN6) in constant table.

Check calculated $\mathrm{Zr}$ and $\mathrm{F} / \mathrm{Zr}$ values.

Unsave all trends. Save trends 3 and 7 on 5 minute interval. Check Bar/status 1 for all loops in manual.

Set CN7 (AN FF), CN18 (FIN AN FE) to 1; set switch 5 (AN FB) to 0. Turn on all pumps: external control, check direction of rotation! Fill reagent lines to addition point

Flow totalizer (DI3) reset, run (force on, unforce)

Turn on switches 3 and 4 (CAS IN 1 for cascade loops)

Set desired final tank level for shutdown event

Turn switches 1 and 2 off

Switch probe recirc loops from calibration solution to process solution.

OPERATION

Switch ANN (loop 1), water (loop 8), and Nitric acid (loop 7) to auto.

Loop 5 is feed. Set to auto and enter flow setpoint. Watch for operation of these pumps.

Turn on switch 1 to enable shutdown once flow events are clear 
Switch loop 2 to auto when a steady valid probe 1 reading is obtained (AI46, PP48),

Turn off switch 3 to forceback loop 2 (ANN first probe feedback cascade middle loop)

Turn on switch 5 to add feedback control to ANN loop.

When a steady valid reading is obtained from probe 2 (AI48, PP59)

switch loop 4 to automatic.

Toggle loop 2 to switch it to remote setpoint.

Turn off switch 4 to forceback loop 4 (ANN OUTER).

Monitor [HF] for approach to setpoint and all loop outputs for onscale operation (not at 1008).

Shutdown at any time by turning switch 2 on.

Watch for feed depletion, product level, and high [HF] alarms.

Watch for reagent depletion.

SHUTDOWN

Normally shutdown occurs at 20 liters actual feed volume (adjusted for SPG) to leave a heel.

Enter 0 setpoint for loop 5 ( $2 \mathrm{r}$ feed). Set to manual and enter 0 output.

Check Bar/status for flow controller outputs $=0$. Switch to manual and set output at zero.

Check that pump rotation has stopped.

Turn off steam.

Record reagent volumes.

Print any desired screens. 

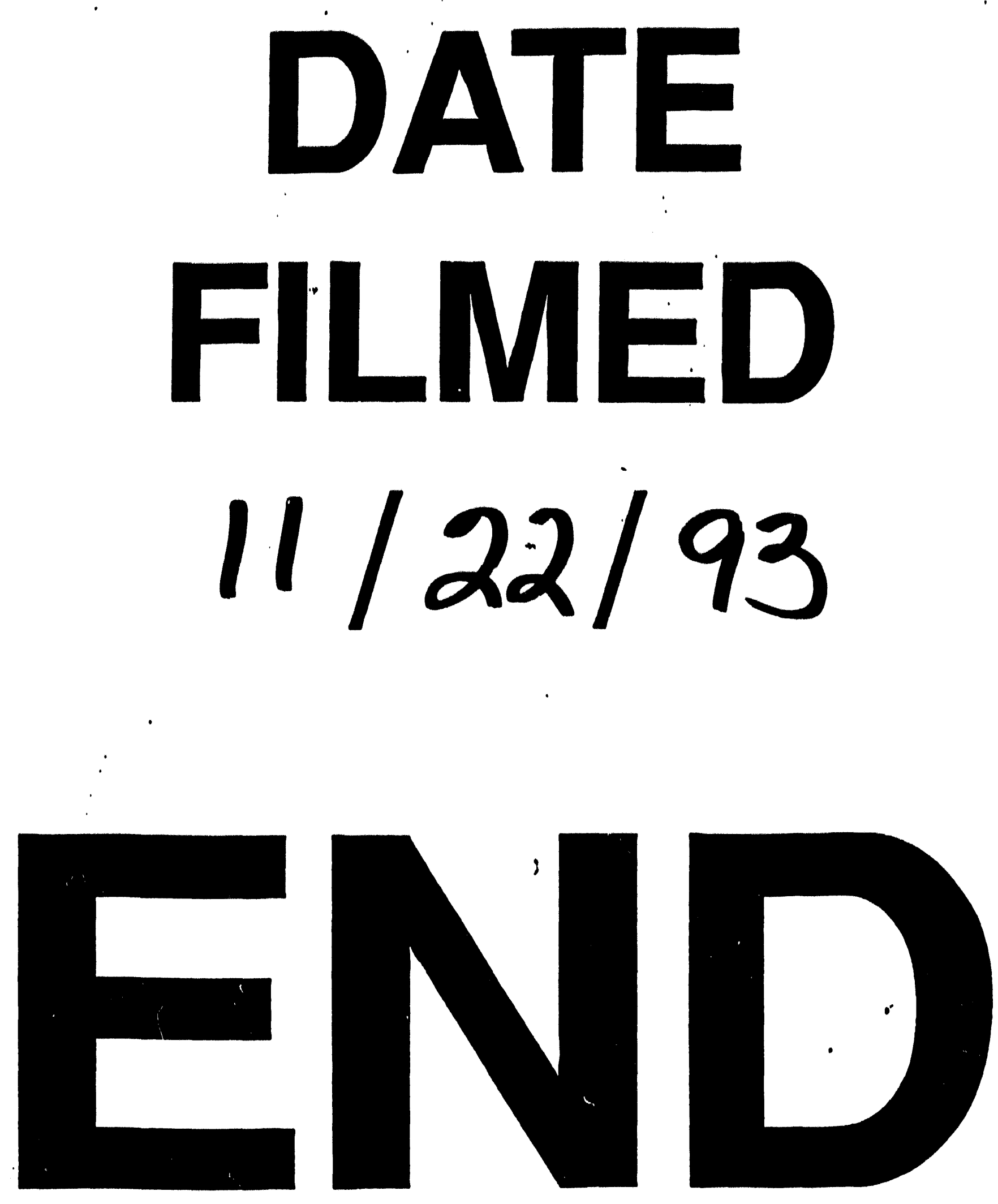


$$
\longrightarrow
$$

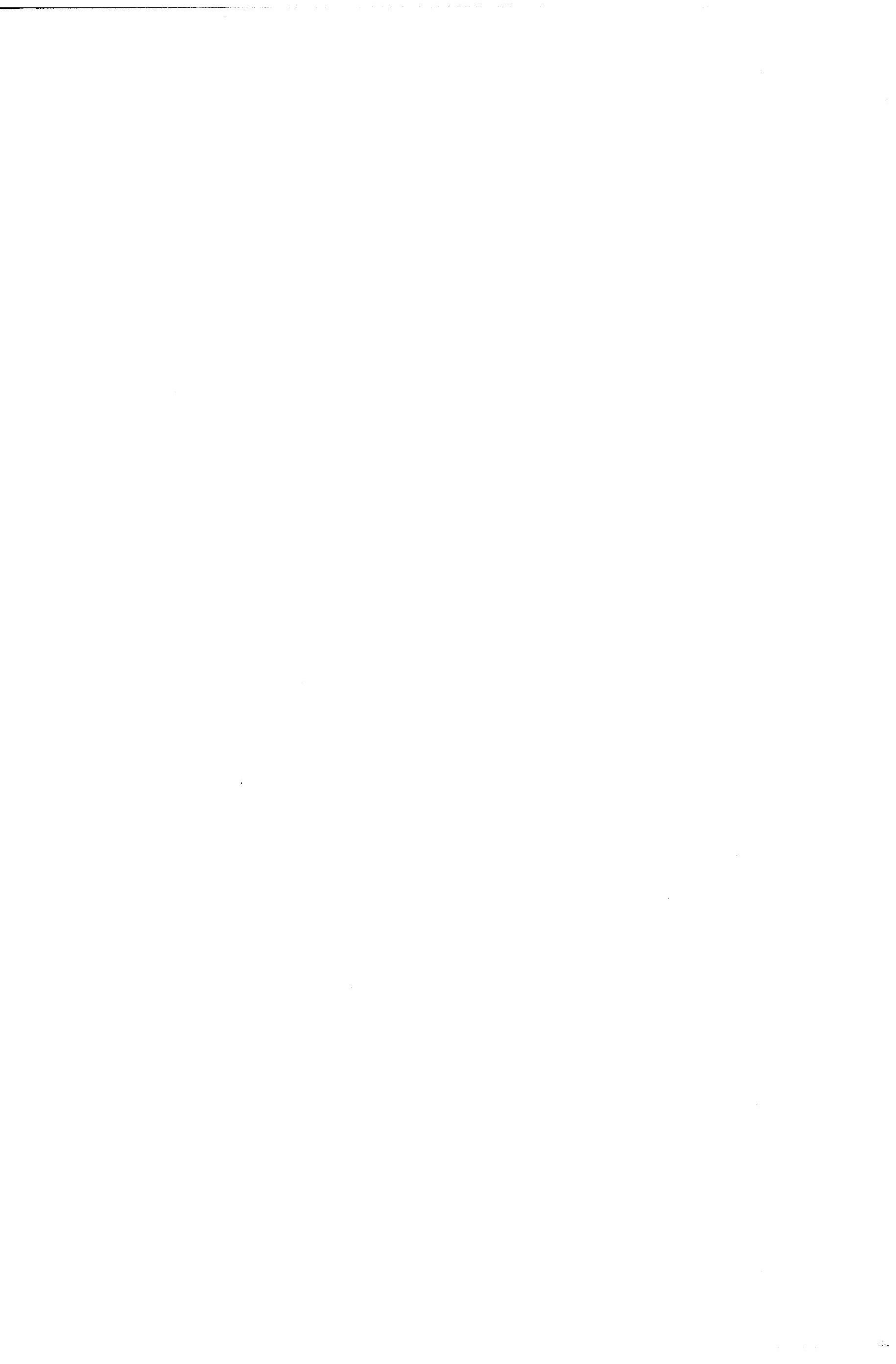

